LAWRENCE LIVERMORE N A T IO N A L LABORATORY

\title{
FY 2004 Annual Report: DOE Project on Heavy Vehicle Aerodynamic Drag
}

R. C. McCallen, K. Salari, J. Ortega, P. Castellucci, C. Eastwood, K. Whittaker, L. J. DeChant, C. J. Roy, J. L. Payne, B. Hassan, W. D. Pointer, F. Browand, M. Hammache, T-Y Hsu, J. Ross, D. Satran, J. T. Heineck, S. Walker, D. Yaste, R. Englar, A. Leonard, M. Rubel, P. Chatelain

November 30, 2004 
This document was prepared as an account of work sponsored by an agency of the United States Government. Neither the United States Government nor the University of California nor any of their employees, makes any warranty, express or implied, or assumes any legal liability or responsibility for the accuracy, completeness, or usefulness of any information, apparatus, product, or process disclosed, or represents that its use would not infringe privately owned rights. Reference herein to any specific commercial product, process, or service by trade name, trademark, manufacturer, or otherwise, does not necessarily constitute or imply its endorsement, recommendation, or favoring by the United States Government or the University of California. The views and opinions of authors expressed herein do not necessarily state or reflect those of the United States Government or the University of California, and shall not be used for advertising or product endorsement purposes.

This work was performed under the auspices of the U.S. Department of Energy by University of California, Lawrence Livermore National Laboratory under Contract W-7405-Eng-48. 


\section{DOE PROJECT ON HEAVY VEHICLE AERODYNAMIC DRAG}

Project Principal Investigator: $R$. C. McCallen

Lawrence Livermore National Laboratory

P.O. Box 808, Livermore, CA 94551-0808

(925) 423-0958; mccallen1@1lnl.gov

Principal Investigator: K. Salari

Co-Investigators: J. Ortega, P. Castellucci, C. Eastwood, K. Whittaker

Lawrence Livermore National Laboratory

P.O. Box 808, Livermore, CA 94551-0808

(925) 424-4635; salari1@,1lnl.gov

Principal Investigators: L. J. DeChant

Co-Investigators: C. J. Roy, J. L. Payne, B. Hassan

Sandia National Laboratories

P.O. Box 5800, MS 0825, Albuquerque, NM 87185-0825

(505)844-4250ljdecha@,sandia.gov

Principal Investigator: $W$. D. Pointer

Argonne National Laboratory

9700 S. Cass Avenue, NE-208, Argonne, IL 60439

(630) 252-1052; dpointer@anl.gov

Principal Investigator: $F$. Browand

Co-Investigators: M. Hammache, T.-Y. Hsu

Aerospace \& Mechanical Engineering, University of Southern California

RRB 203, Los Angeles CA 90089-1191

(213) 740-5359; e-mail: browand@spock.usc.edu

Principal Investigator: $J$. Ross

Co-Investigators: D. Satran, J.T. Heineck, S. Walker, D. Yaste

NASA Ames Research Center

MS 260-1, Moffett Field, CA 94035

(650)604-6722; jcross@mail.arc.nasa.gov

Principal Investigator: $R$. Englar

Georgia Tech Research Institute

ATASL, CCRF, Atlanta, GA 30332-0844

(770) 528-3222; bob.englar@gtri.gatech.edu 
Principal Investigator: A. Leonard

Co-Investigator: M. Rubel, P. Chatelain

California Institute of Technology

1200 East California Blvd. MC 301-46, Pasadena, CA 91125

(626) 395-4465; tony@galcit.caltech.edu

\section{Technology Development Manager: Sid Diamond}

202-586-8032, sid.diamond@ee.doe.gov

Technical Program Manager: Jules Routbort

630-252-5065, routbort@,anl.gov

Contractor: Lawrence Livermore National Laboratory,Sandia National Laboratories, Argonne National Laboratory, NASA Ames Research Center, Georgia Tech Research Institute, University of Southern California, California Institute of Technology

Contract No.: W-7405-ENG-48, DE-AC04-94AL85000, W-31-109-ENG-38, DE-AI0199EE50559, DE-AC03-02EE5069, DE-AC03-98EE50512, DE-AC03-98EE50506

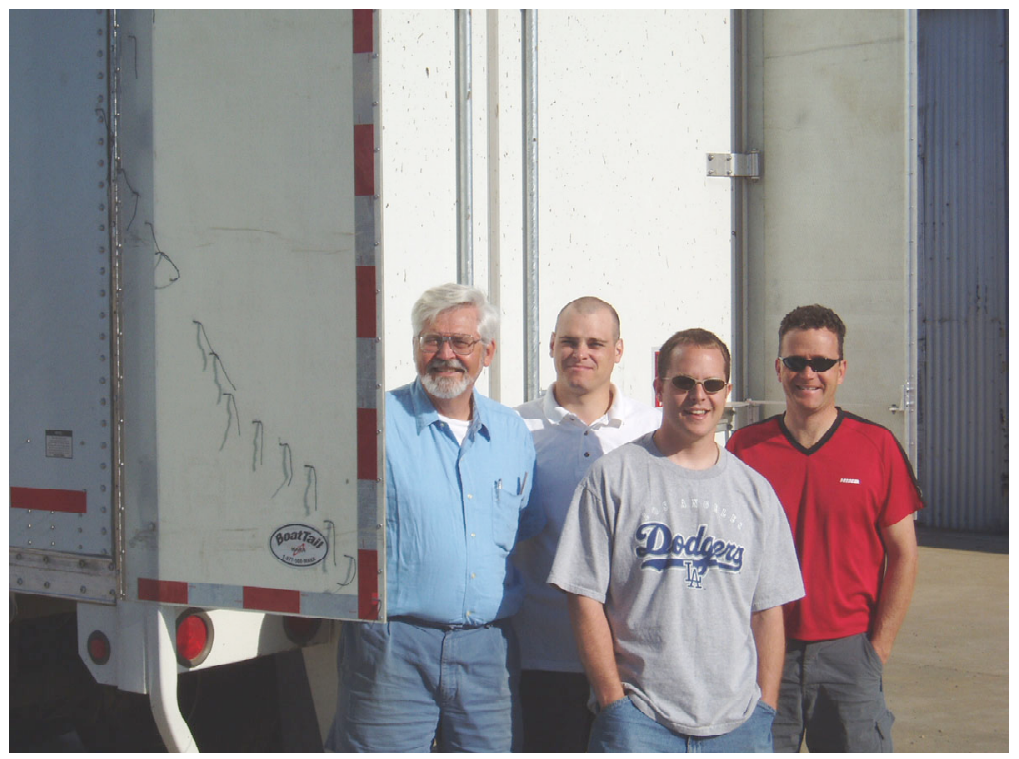

Consortium members road testing base-flaps at Crows Landing, California.

Charles Radovich, USC

Fred Browand, USC Scott Johnston, PATH

Mathieu Boivin, Norcan Aluminum 


\section{Objective}

- Provide guidance to industry in the reduction of aerodynamic drag of heavy truck vehicles.

- Establish a database of experimental, computational, and conceptual design information, and demonstrate potential of new drag-reduction devices.

\section{Approach}

- Develop and demonstrate the ability to simulate and analyze aerodynamic flow around heavy truck vehicles using existing and advanced computational fluid dynamics (CFD) tools.

- Through an extensive experimental effort, generate an experimental data base for code validation.

- Using experimental data base, validate computations.

- Provide industry with design guidance and insight into flow phenomena from experiments and computations.

- Investigate aero devices (e.g., base flaps, tractor-trailer gap stabilizer, underbody skirts and wedges, blowing and acoustic devices), provide industry with conceptual designs of drag reducing devices, and demonstrate the full-scale fuel economy potential of these devices.

\section{Accomplishments}

- The Program has demonstrated several concepts and devices which meet the $25 \%$ drag reduction goal.

- Insight from experiments and experimental data base has provided clear guidance to industry on reliable, predictable experimental techniques.

- Computational results provide clear guidance and caution warnings on the use of steady Reynoldsaveraged Navier Stokes (RANS) models for CFD simulations.

- Investigated aerodynamics of filled and empty rail coal cars and designed devices predicted to produce drag reductions of $10 \%$ for empty cars.

\section{Future Direction}

- Continue to develop and evaluate drag reducing conceptual designs and encourage and work with industry to road test the most promising drag reducing devices.

- Continue experimental data reduction and analysis for the generic conventional model (GCM).

- Continue computations of flow around GCM, compare to experimental data, perform analyses, and provide guidance to industry on use of unsteady RANS and hybrid RANS/Large-Eddy Simulation (LES) methods.

- Develop and use an apparatus for studying wheel and tire splash and spray, pursuing ways to minimize this road safety hazard.

- Investigate air flow around rotating tires for improved brake cooling, as well as drag reduction.

- Collaborate with DOE Industrial Consortium who will be conducting fleet tests of advanced aerodynamic drag reduction devices. Schedule industry site visits and meetings to share findings and encourage consideration of effective design concepts for road testing.

- Leverage Program work and seek funding from other agencies. 


\section{Introduction}

A modern Class 8 tractor-trailer can weigh up to 80,000 pounds and has a wind-averaged drag coefficient around $C_{D}=0.6$. The drag coefficient is defined as the $\mathrm{drag} /$ (dynamic pressure $\mathrm{x}$ projected area). The higher the speed the more energy consumed in overcoming aerodynamic drag. At 70 miles per hour, a common highway speed today, overcoming aerodynamic drag represents about $65 \%$ of the total energy expenditure for a typical heavy truck vehicle. Reduced fuel consumption for heavy vehicles can be achieved by altering truck shapes to decrease the aerodynamic resistance (drag). It is conceivable that present day truck drag coefficients might be reduced by as much as $50 \%$. This reduction in drag would represent approximately a $25 \%$ reduction in fuel use at highway speeds. An estimated total savings of $\$ 1.5$ billion per year (pre-2004 fuel prices) can be recognized in the United States alone for just a $6 \%$ reduction in fuel use. This reduction represents $1 \%$ of all fuel use in the United States.

The project goal is to develop and demonstrate the ability to simulate and analyze aerodynamic flow around heavy truck vehicles using existing and advanced computational fluid dynamics (CFD) tools. Activities also include an extensive experimental effort to generate data for code validation and a design effort for developing drag reducing devices. The final products are specific device concepts that can significantly reduce aerodynamic drag, and thus improve fuel efficiency, in addition to an experimental data base and validated CFD tools. The objective is to provide industry with clear guidance on methods of computational simulation and experimental modeling techniques that work for predicting the flow phenomena around a heavy vehicle and addon drag reducing devices. Development of effective drag reducing devices is also a major goal.

The following reports on the findings and accomplishments for fiscal year 2004 in the project's three focus areas

- Drag reduction devices

- Experimental testing

- Computational modeling
A summary is given in the introduction portion of this report and detailed reports from each participating organization are provided in the appendices. Included are experimental results and plans by NASA, USC, GTRI, and LLNL in Appendices A through D. The computational results from LLNL and SNL for the integrated tractor-trailer benchmark geometry called the Ground Transportation System (GTS) model and trailer wake flow investigations are in Appendices $\mathrm{D}$ and $\mathrm{E}$, from ANL for the Generic Conventional Model (GCM) in Appendix F, by LLNL for the tractor-trailer gap flow investigations in Appendix $\mathrm{D}$, and turbulence model development and benchmark simulations being investigated by LLNL and Caltech in Appendices D and G. USC is also provides field test results for the base flap device (Appendix B), GTRI continues their investigation of a blowing device (Appendix C), and LLNL presents results for base skirts and wedges (Appendix D).

\section{Drag Reduction Devices}

There are three areas identified for aero drag reduction and several drag reduction devices have been investigated

- Tractor-Trailer Gap

Stabilizing devices, cab extenders

- Wheels/Underbody

Skirts/lowboy trailer $\left(\Delta \mathrm{C}_{\mathrm{D}} \sim 0.05\right)$, splitter plate

- Trailer Base

Boattail plates $\left(\Delta \mathrm{C}_{\mathrm{D}} \sim 0.05\right)$, base flaps $\left(\Delta \mathrm{C}_{\mathrm{D}} \sim\right.$ $0.08)$, rounded edges, and pneumatics

\section{Overview of Accomplishments}

The Program has demonstrated several concepts and devices which meet the $25 \%$ drag reduction goal. Specific devices have addressed base, gap, and underbody drag reduction. Use of a simple base flap at the trailing edge of the trailer, side extenders or splitter plate at the tractor-trailer gap, and a skirt or a simple short underbody wedge should provide drag reduction exceeding $25 \%$. At highway speeds, fuel savings around $12 \%$ should be recognized for a $25 \%$ reduction in drag. 
This would represent a savings of $\$ 3$ billion/year in the United States (pre-2004 fuel prices).

The highly successful testing program has provided detailed data for computational validation, guidance on device concepts, and established wind-tunnel testing guidelines. The detailed data exceeds what is typically available for careful code validation in a relatively complex flow and is thus of interest to the general fluid dynamics/aerodynamics research and development community. The state-of-the-art in Particle Image Velocimetry (PIV) was significantly advanced in the efforts at the NASA wind tunnels. With the Ground Transportations System (GTS) model in the 7 -ft $\times 10-\mathrm{ft}$ wind tunnel, NASA succeeded in being one of the first to use a three-dimensional (3D) PIV system in a production wind tunnel. To use PIV in the 12-ft pressure wind tunnel with the Generic Conventional Model (GCM), a new and innovative approach that provided remote control of the PIV system was developed. With this remote system, the tunnel was not opened-a costly and time consuming procedure - to reposition cameras.

The computational flow modeling has provided guidance in model definition, mesh refinement, and choice of turbulence model for heavy vehicles. Computations have been used for both the evaluation of flow physics and to guide the conceptual design of devices. For example, it was demonstrated computationally that a splitter plate that partially closes the tractor-trailer gap is adequate to maintain the desired reduced drag, symmetric flow condition and avoid gap blow through. Previous designs assumed that full gap closure from the tractor to the trailer was necessary.

The Program has successfully established industry contacts and collaborations and international recognition in the academic community. The $I^{\text {st }}$ International Conference on the Aerodynamics of Heavy Vehicles: Trucks, Busses and Trains, which was lead by the DOE Aero Team, attracted world renowned researchers and developers from academia, along with significant industry interest and participation. It should also be emphasized that by combining the best of academia and government lab capabilities, technical developments have been leveraged across programs within DOE Labs, NASA, and university programs, while delivering the DOE
Heavy Vehicle Program milestones. Examples are the progress in the state-of-the-art in 3D PIV, advances in turbulence modeling with the use of hybrid RANS and LES models for efficient and accurate flow modeling, and the use of broadcast fuel rates during real-time, full-scale testing. The Program achievements also include the Team's many publications and record-of-inventions or patents that have resulted from the DOE Heavy Vehicle Program work.

\section{Future Plans}

Future new areas being investigated are wheel and wheel well aerodynamics related to brake cooling, tire splash and spray, and an entire new related area of investigation involving the evaluation of coal car aerodynamics with the objective of identifying drag reduction devices for filled and empty cars.

The Team will continue with their computational effort while enhancing their fullscale testing effort in collaboration with fleet owners and manufacturers. Substantial efforts to establish contacts with the fleets are planned. For example, the Team representative, Jim Ross, was invited to participate in a panel at the TMA meeting in Nashville Tennessee this September. The focus topic of the panel is the recognized increase in fuel use during the cold weather season. Jim used this opportunity to share the Team's findings with fleet owners and operators and seek their feedback on ways to get aero device technology on the road.

To successfully get aerodynamic devices on the road, full-scale testing in collaboration with fleet owners and operators is needed. Testing locations that have been thus far utilized by the Team are the TRC in Ohio and Crow's Landing in California. Both controlled and long road tests are needed at speeds at or exceeding 65 miles per hour.

Computations of rotating wheels and investigating the influence of underbody flow are planned and are recognized areas of interest to industry. This effort is in addition to moving forward on full-vehicle simulations with advanced models. Unsteady RANS and hybrid LES/RANS modeling of the full GCM vehicle with comparison and analysis of the 12-ft NASA Pressure Wind Tunnel data is planned. It is 
important to determine if unsteady RANS and hybrid RANS/LES turbulence modeling can capture primary flow features. Guidelines for steady RANS have been openly shared and are available to industry. Unsteady RANS and hybrid models need to be assessed for grid sensitivity and boundary conditions. This will provide specific guidelines for computations with advanced models and will assist in the further conceptual design of drag reduction devices and an integrated vehicle.

Tractor manufacturers are also interested in computational results for specific commercial tools, but it should be noted that guidelines for use of specific turbulence modeling approaches is not dependent on choice of computational tool. Currently, the National Lab participants are utilizing commercial and NASA codes, as well as their own in-house tools. There are advantages to each. For the addition of new models and for response to $R \& D$ issues, especially on large parallel machines for investigating model performance, the NASA and in-house tools provide the quickest and most flexible situation. However, for geometry and mesh generation, the commercial tools tend to provide some desirable options.

It is recognized that further fuel savings are possible and vehicle safety can be enhanced by leveraging the accomplishments of the DOE Aero Team to investigate an integrated heavy vehicle system. The effect of aerodynamics on brake cooling and engine cooling will be considered. Air control for improved braking and engine performance is currently a high priority for industry. Also, the initiated efforts in wheel, tire, and vehicle splash and spray will continue. This splash and spray investigation will provide an understanding of this multiphase flow phenomena, thus leading to conceptual designs for mitigation of splash and spray for improved vehicle and highway safety. Published research and development in the open literature appears to be void of information in this area of interest.

The Team is also planning to continue their pursuit to improve aerodynamics and reduce fuel use areas with similar flow regimes to that of heavy vehicles. This year's experiments and computations of railway coal cars have demonstrated the substantial increase in drag from full to empty railcars. The aerodynamic drag of an empty railcar in the wind tunnel is $32 \%$ and $42 \%$ at 0 and 10 degrees yaw, respectively, over that for a full railcar. We plan to continue working with contact, Jim Hart, of Johnstown America Corporation, Johnstown, Pennsylvania for guidance in conceptual designs that are automatic and durable for the 20 plus year life of a coal car. Experiments and computations have been used for the smart design of drag mitigating devices. These conceptual designs will condition the flow so that the empty car will mimic the flow of a full car, providing substantial fuel savings. 


\title{
APPENDIX A
}

\section{A Study of Reynolds Number Effects and Drag-Reduction Concepts on a Generic Tractor-Trailer}

\author{
Principal Investigator: Bruce L. Storms \\ AerospaceComputing, Inc. \\ $M / S / 260-1$ \\ NASA Ames Research Center \\ Moffett Field, CA 94035 \\ (650)604-1356,fax: (650)604-4511,e-mail:bstorms@mail.arc.nasa.gov
}

Field Project Manager: James C. Ross

NASA Ames Research Center

$M / S / 260-1$

Moffett Field, CA 94035

(650)604-1356, fax: (650)604-4511, e-mail: james.c.ross@nasa.gov

Technology Development Area Specialist: Sidney Diamond (202) 586-8032; fax: (202) 586-1600; e-mail: sid.diamond@ee.doe.gov

Technical Program Manager: Jules Routbort

(630)252-5065; fax: (630)252-4289; email: routbort@anl.gov

Participants

Dale R. Satran, James T. Heineck, Stephen M. Walker

NASA Ames Research Center

Contractor: NASA Ames Research Center

Contract No.: DE-AI01-99EE50559

NASA's effort consists of tow experimental focus areas:

- A Study of Reynolds Number Effects and Drag-Reduction Concepts on a Generic Tractor-Trailer Objective

- An Experimental Study of Aerodynamic Drag of Empty and Full Coal Cars

The following describes the objective, approach, accomplishments, and future direction for each of these focus areas.

\section{A. A Study of Reynolds Number Effects and Drag-Reduction Concepts on a Generic Tractor-Trailer Objective}

\section{Objective}

- To investigate Reynolds-number effects on the flow field and resulting aerodynamic forces generated by a 1:8-scale model of a class- 8 tractor-trailer configuration

- To provide quality experimental data on a simplified tractor-trailer geometry for CFD validation. 


\section{Approach}

- To vary the total pressure of the wind tunnel thereby varying the Reynolds number from 500,000 to fullscale values over 6 million based on trailer width.

- Measure the forces and momentum surface, pressure distribution, and off-body flow. Measurements were made at various yaw angles to study the influence of crosswind and to calculate wind-averaged drag coefficients.

- Several drag-reduction concepts were studied in order to document their potential benefit as well as their Reynolds-number sensitivity.

\section{Accomplishments}

- CFD validation data is now available for use by interested industry and government researchers

- Reynolds number effects were found to be relatively small above a value of $\sim 1$ million. Care should be taken in interpreting smaller-scale data

- The results of the study were presented at the $34^{\text {th }}$ AIAA Fluid Dynamics Conference and Exhibit in Portland, Oregon on July 1, 2004 (paper number AIAA-2004-2251)

\section{Future Direction}

- Additional drag-reduction devices will be examined for under-body flow control/drag reduction

- Results from this study will be made more widely available via CD/DVD distribution to interested parties

\section{Introduction}

For a typical heavy vehicle at a highway speed of $70 \mathrm{mph}$, the energy required to overcome aerodynamic drag is about $65 \%$ of the total expenditure (which also includes rolling friction, transmission losses, and accessories). By altering the vehicle shape, it has been estimated that modern truck drag coefficients may be reduced by up to $50 \%$ resulting in an annual national fuel savings of three billion gallons. This large potential savings coupled with increasing fuel costs have spurred renewed interest in heavy-vehicle aerodynamics.

Recently, a series of experimental and computational studies have been funded by the Department of Energy. With the goal of CFD validation, the experimental efforts have focused on simplified geometries at 1:8-scale and below. Early experiments focused on the simplified geometry of the Ground Transportation System (GTS) model representative of a class- 8 tractor-trailer with a cabover-engine design. A 1:8-scale GTS model with no tractor-trailer gap and no wheels was first studied with the addition of several ogival boattails and slants to the base of the trailer. The largest overall drag reduction of $10 \%$ was obtained by an 8 -ft ogive configuration (full scale). The addition of boattail plates to the same model resulted in a $19 \%$ drag reduction and PIV measurements behind the trailer document a significant reduction in the wake size due to the flow turning provided by the plates. Variation of the tractor-trailer gap on a 1:15-scale model at zero yaw revealed relatively constant drag on the tractor while the trailer drag increases by a factor of three as the gap increases from zero to $1.55^{*} \sqrt{ }$ A. Reynolds-averaged Navier-Stokes computations of this geometry include both grid-size and turbulence-model studies.

The Generic Conventional Model of the current study was previously investigated at a Reynolds number of 1.1 million in the NASA-Army 7- by 10Ft Wind Tunnel. The results include forces and moments, surface pressures, and 3-D particle-image velocimetry. Measurements of two tractor-trailer gaps (40 and 80 inches full scale) indicated significantly greater drag for the larger gap at low yaw angles (between $\pm 4 \mathrm{deg}$ ) and reduced drag at higher angles. Several drag-reduction concepts were investigated including tractor side extenders, boattail plates, and a trailer belly box. Comparisons of PIV data were presented in the tractor-trailer gap with and without side extenders and in the trailer wake with and without boattail plates. 


\section{Experimental Setup}

The investigation was conducted in the 12-Foot Pressure Wind Tunnel located at NASA Ames Research Center. This facility can be pressurized from 0.25 to 6 atmospheres at Mach numbers from 0.1 to 0.5 . The test section has a circular cross section $12 \mathrm{ft}$ in diameter with four 4 -ft wide flat surfaces centered about the horizontal and vertical centerlines. A ground plane was installed 21 inches above the tunnel floor providing a flat surface $10 \mathrm{ft}$ wide and $18 \mathrm{ft}$ long. Pressure taps were located on both the ground plane ( 2 rows of 64 taps) and the test-section walls (8 rows of 30 taps). A fairing was installed to isolate the model-support hardware from the air stream, and speed-correction probes were used to correct the facility speed due to the blockage of the ground plane and fairing. There was also an additional pitot-static probe installed on the upper left ceiling to measure the free-stream conditions in the test section. All of the data presented is referenced to the Mach number based on a wall tap at location $6.17 \mathrm{ft}$ forward of the center of rotation at an azimuth of $60 \mathrm{deg}$ from vertical (two o'clock looking downstream).

A photograph of the GCM baseline configuration installed in the wind-tunnel test section is shown in Fig. 1. This 1:8-scale model is representative of a generic class- 8 tractor-trailer with the engine in front of the cab. Designed for CFD validation, the model includes a number of geometry simplifications in order to facilitate grid generation and avoid the associated flow complexities. In particular, no effort was made to duplicate the complex geometry of the undercarriage of either the tractor or trailer (both were approximated by flat surfaces). Similarly, the wheel wells of the tractor were not modeled and only the portion of the wheels below the tractor lower surface were included. Also, the tractor geometry (designed by the Calmar Research Corp.) is a streamlined shape representative of a modern tractor design while omitting most small-scale surface details and flowthrough components. The trailer measures $45 \mathrm{ft}$ in length (full scale) with rounded front vertical edges (8-in full-scale radius). The tractor-trailer gap for this study was held constant at the full-scale equivalent of 40 inches. The GCM was attached to the model-support hardware with four vertical posts that were 1.75 inches in diameter. The four posts were non-metric with 0.030 inches of clearance as they passed through the trailer floor. The model was mounted with its wheels 0.15 inches above the ground plane and centered laterally in the tunnel. The center of rotation of the model was located 54.36 inches aft of the tractor front bumper. The model frontal area of $1.6623 \mathrm{ft}^{2}$ gives a solid blockage of $1.5 \%$.

The overall model loads were measured with a six-component internal balance that was mounted inside the trailer. The manufacturer-specified accuracy of the internal balance in the axial (drag) direction was $\pm 1 \mathrm{lb}$, but repeat runs indicated the experimental uncertainty to be on the order of \pm 0.5 $\mathrm{lb}$. The tractor was suspended from the trailer through a set of flexures and 2 load cells that measure the drag and yawing moment of the tractor alone. The specified accuracy of the load cells was $\pm 0.45 \mathrm{lb}$. The model was instrumented with 200 pressure taps on the tractor and 276 taps on the trailer. There were also 12 unsteady pressure transducers mounted on the tractor rear surface, trailer front surface, and the trailer rear surface. A three-component particle image velocimetry (PIV) system was used to obtain horizontal-plane velocity measurements in the tractor-trailer gap. Details of the PIV system installation are presented in Ref. 12. Pressure measurements will be documented in a future report.

The model was yawed through a range of angles between \pm 14 degrees. Except where noted, all data were acquired at a Mach number of 0.15 which allowed for Reynolds number studies with no Machnumber effects. With the tunnel pressurized to six atmospheres, the Reynolds number was over 6 million based on the trailer width which is comparable to a full-scale truck driving at $75 \mathrm{mph}$. At one atmosphere, the Reynolds number was 1 million based on the trailer width which is comparable to a full-scale truck driving at $15 \mathrm{mph}$.

Various add-on drag-reduction devices were tested on the bases of both tractor and trailer as well as on the trailer under-carriage. In this report, results will be presented for tractor side and roof extenders, trailer boattail and base flaps, and trailer belly box and skirts. Details of each device will accompany the discussion of the associated results.

\section{$\underline{\text { Results and Discussion }}$}

The results presented below detail the body-axis axial force (drag) coefficient for the tractor-trailer 
combination and its components. This drag coefficient represents the force along the axis of the vehicle in the direction of travel. The internal balance also provided lift, side force, and moment measurements which will not be discussed. With the objective of CFD validation, no wall corrections were applied to the data and all coefficients were calculated based on the static pressure at a known point in the test section (as detailed above). Without wall corrections, the computed drag coefficients will differ from those of the equivalent model in free air. However, the measured differences between configurations should be representative of the effects of the associated geometric modifications.

The drag data presented herein were acquired for increasing yaw angle unless otherwise noted. Using the variation of drag with yaw angle, windaveraged drag coefficients were computed using the SAE Recommended Practice of Ref. 13. This practice assumes that the mean wind speed in the United States of $7 \mathrm{mph}$ has an equal probability of approaching the vehicle from any direction. This mean wind speed and the vehicle velocity were used to calculate a weighted average of the drag coefficient at various yaw angles. The windaveraged drag coefficients reported in this paper were computed for a highway speed of $55 \mathrm{mph}$.

\section{A. Baseline Configuration}

The baseline geometry for this study is representative of a modern tractor design with the standard aero package less the side or roof extenders. Yaw angle sweeps for increasing and decreasing angle revealed significant hysteresis in the resulting drag measurements (Fig. 2). Since the drag curve of a simplified geometry with no gap is relatively continuous, the discontinuities at high yaw angles are likely due to changes of the flow structures within the tractor-trailer gap. The effect of Reynolds number is most evident at the yaw angles greater than 8 deg where the peak drag and hysteresis are notably different. At lower yaw angles, however, the differences are relatively small and the hysteresis paths are nearly duplicated. For a mean crosswind of $7 \mathrm{mph}(-7.2<\psi<7.2)$ as shown in Fig. 2, the wind-averaged drag coefficients at Reynolds numbers of 1 and 6 million ( 0.582 and 0.578 , respectively) differed by less than $1 \%$.

A three-component PIV system was employed for velocity measurements in the tractor-trailer gap. The velocity vector maps (Figs. 3-5) are averages of
100 discrete measurements acquired at $2 \mathrm{~Hz}$ in intermittent bursts (due to computer limitations) over a period of three minutes. Although data were acquired at $1 / 4,1 / 2$, and $3 / 4$ trailer heights, the results presented in this report are limited to the topmost location. In these figures, the direction and magnitude of the vectors indicate the in-plane velocities while the color map indicates the out-ofplane (vertical) velocity. The velocity vector maps for zero yaw (Fig. 3) reveal two counter-rotating recirculation regions in the gap and suggest minimal sensitivity to Reynolds number. The yaw angle of $-10 \mathrm{deg}$ (Fig. 4), however, is in the hysteresis region of the drag curves where there are significant differences between two Reynolds numbers. Both measurements were obtained for decreasing yaw angle that correspond to the upper curves as indicated in Figure 2. Similar to the zero-yaw case, the flowfield at $\mathrm{Re}=5$ million exhibits two recirculation regions, but they are strongly asymmetric and with much higher velocities. At Re $=1$ million, only the tight recirculation on the windward (left) side is present and there are significantly greater crossflow and downward velocities compared to the higher Reynolds number. The corresponding drag coefficient for $\mathrm{Re}=1$ million is $2 \%$ higher than that for $\mathrm{Re}=5$ million. The lower measurement locations revealed similar flow structures to the topmost height, but with reduced Reynolds-number sensitivity.

The PIV data also provide insight into the dragcurve hysteresis. A look at two neighboring yaw angles near -10 deg reveal drastically different flowfields at one Reynolds number (Fig. 5). As indicated on the drag curves (Fig. 2), these two measurements correspond to yaw angles of $-10 \mathrm{deg}$ (decreasing) and $-10.5 \mathrm{deg}$ (increasing) at $\mathrm{Re}=1$ million. For the yaw angle of $-10.5-\mathrm{deg}$, the windward recirculation region is absent and the velocities in the gap are significantly lower than the -10-deg case. It is hypothesized that lower gap velocities yield higher pressures on the back of the cab (less drag) and a smaller separation region on the leeward (right) side of the truck (not visible in PIV images). As a result, the drag of the -10.5 -deg case is $28 \%$ lower than that of the -10 -deg case.

\section{B. Side and Roof Extenders}

Similar to the components of a modern tractor aero package, side and roof extenders were attached to the rear of the tractor as shown in Figure 6. The 
extenders were $1 / 8$-in thick (model scale) with four different lengths ranging from $30 \%$ to $60 \%$ of the tractor-trailer gap. For a length of $60 \%$ gap (as shown), the total drag coefficient as a function of yaw angle (Fig. 7) illustrates the dramatic effect of the extenders. At yaw angles less than $2 \mathrm{deg}$, the drag reduction is minimal while at higher angles the reduction increases dramatically to $35 \%$ at $10 \mathrm{deg}$. At a Reynolds number of 6 million, wind-averaged drag coefficient of 0.422 for the $0.6 \mathrm{~g}$ extenders was $27 \%$ lower than that of the baseline without extenders. The low Reynolds-number measurements were marginally higher than those of the full-scale Reynolds number until about 10 deg yaw the curves cross and the low-Re drag drops off at the highest yaw angle of $14 \mathrm{deg}$. PIV data for this model with extenders were acquired previously in the NASA Ames 7- by 10-Ft Wind Tunnel at a Reynolds number of 1 million. The results indicate a significant reduction in the gap cross flow due to the presence of the side and roof extenders.

The wind-averaged drag reduction provided by the extenders is plotted as a function of extender length in Figure 8. For the four extender lengths tested between $30 \%$ and $60 \%$ gap width, there is a consistent trend of increasing drag reduction with increasing extender length. More specifically, the drag reduction increases from $25 \%$ to $27 \%$ for an increase in extender length from $30 \%$ gap to $60 \%$ gap. Although greater extender lengths may be impractical from an operational standpoint, this data suggests that additional drag reduction may be obtained by completely blocking the gap crossflow. As demonstrated in Ref. 2, a centerline gap seal can be more effective than the side extenders of a standard tractor aero package.

To determine the effect of Reynolds number, the facility total pressure was varied while the Mach number was held constant. The change in windaveraged drag coefficient with Reynolds number (Fig. 9) reveals differing sensitivities for the baseline and extender configurations. In this figure, the error bars on the baseline data points show the magnitude of experimental uncertainty due to both measurement resolution and repeatability. Error bars were of the same magnitude for the extender configuration, but were omitted from the figure for clarity. For the baseline, the drag coefficient is observed to increase by an average of $0.006(1 \%)$ for Reynolds numbers less than 4 million. The extender configuration, however, does not indicate a significant increase until below 3 million with a dramatic increase, close to $0.03(6.9 \%)$, at $\mathrm{Re}=$ 500,000 . The drag curves for the extender configuration at several Reynolds numbers (Fig. 10) illustrate a significant increase in drag that increases with crossflow at $\operatorname{Re}=500,000$. This is likely due to flow variations in the vicinity of the trailer leadingedge curvature which is more sensitive to Reynolds number than sharp corners.

\section{Aerodynamic Boattail Plates}

Since the side and roof extenders are firstgeneration drag-reduction devices common to most modern tractor aero packages, the effect of the boattail plates (and all subsequent devices) will be measured relative to the extender configuration. Aerodynamic boat-tailing devices have several different variations, but boattail plates typically refer to panels mounted perpendicular to the trailer base and inset from the edges of the trailer. In this case, the same boattail plates that were studied on the simplified GTS model ${ }^{5}$ were applied to the rear of the GCM trailer as shown in Figure 11. The plates extended 3.75 in from the end of the trailer and were inset from the sides and top of the trailer by 0.625 in. The bottom plate was mounted flush with the bottom of the trailer.

Relative to the side and roof extenders, the boattail plates significantly reduced the drag by a relatively constant margin between $\pm 10 \mathrm{deg}$ (Fig. 12). At $\operatorname{Re}=6$ million, the wind-averaged drag coefficient was 0.364 which is $13.7 \%$ less than the extenders-only configuration. Relative to the high Reynolds-number case, the drag curve for the boattail plates at $\operatorname{Re}=1.1$ million exhibited the same roll off at high yaw angles as that of the side extenders alone. The results were mixed at lower angles with the low-Re curve lower than the high-Re curve at negative yaw angles and higher at some positive yaw angles. These mixed results are evident in the resulting wind-averaged drag coefficients which differ by only $0.6 \%$.

\section{Trailer Base Flaps}

Another method of aerodynamic boat-tailing is what will be referred to as base flaps. In this embodiment, the panels are attached to the edges of the trailer base and angled inward. In the current study, measurements were made for a base-flap length of 3.125 inches ( 25 inches full scale) at 
angles ranging from zero to $28 \mathrm{deg}$. The installation photo (Fig. 13) shows the base flaps with a $20-\mathrm{deg}$ deflection mounted on the rear of the trailer. Note that the linkages connecting the flaps to the base were designed for easy angle change and are not representative of the full-scale hardware.

The effect of the base flaps on the total drag coefficient is presented in Figure 14 for a flap angle of $16 \mathrm{deg}$. Relative to the side and roof extenders alone, the addition of the base flaps provide significant drag reduction that marginally increases with yaw angle. The Reynolds-number sensitivity of the base flaps is minimal for most yaw angles between $\pm 10 \mathrm{deg}$. As in the previous configurations, the drag for $\mathrm{Re}=1.1$ million rolls off at the higher yaw angles while the drag at full-scale Reynolds number continues to increase.

The effect of base-flap angle on wind-averaged drag reduction is presented in Figure 15. Different symbols are used to indicate the data from two separate wind-tunnel entries (four months apart) of the same model in the NASA Ames 12-Ft Pressure Wind Tunnel. The lower Reynolds number on the second entry was due to limitations of the coincident PIV measurements. Although there is an unexplained offset in the two curves (marginally larger than the experimental uncertainty), the trends indicate that the optimum base-flap angle is around $20 \mathrm{deg}$. The wind-averaged drag coefficient with $20-$ deg base flaps is 0.340 which is $19.4 \%$ less than the extender configuration and $6.6 \%$ less than the boattail-plate configuration. Previous small-scale experiments $^{2}$ at a Reynolds number of 1.25 million yielded an optimum base-flap configuration with an angle of 15 degrees and a full-scale panel length of 20 inches. The difference between these two experiments is likely due to the differences in the base-flap lengths since the Reynolds-number effects were observed to be minimal. However, the effect of Reynolds number might be more significant at higher flap angles where the flow reattachment on the base flaps is more sensitive.

\section{E. Trailer Belly Box and Skirts}

Several drag-reduction concepts were studied with the goal of reducing the lateral flow under the trailer. Similar to previous studies, trailer side skirts consisted of flat panels extending downward from the sides of the trailer between the end of the tractor bed and the front of the trailer wheels. The full-skirt configuration extended to the rear of the trailer covering the trailer wheels and also included lateral panels at the trailer base and behind the tractor bed. The trailer belly box (Fig. 16), so named because of its resemblance to the design of moving trailers, was identical to the full-skirt configuration with the addition of a horizontal surface at the bottom of the skirt to form a box on the undercarriage of the trailer. All of these configurations had a full-scale ground clearance of 11.8 inches.

The varying effect of the trailer belly box and skirts on the overall drag is presented in Figure 17 for $\operatorname{Re}=6.3$ million. Relative to the extender-only case, the trailer belly box was the most effective with a wind-averaged drag reduction of $11.8 \%$. The side skirts were somewhat less effective with a drag reduction of $6.2 \%$ while the full-skirt configuration increased the wind-averaged drag by $3.8 \%$. The increase in drag of the full-skirt configuration is likely due to the cavity flow that forms inside the skirt. The wind-averaged drag coefficients for these three cases were $0.372,0.396$, and 0.438 , respectively. Due to time limitations, a Reynoldsnumber sensitivity study was not performed for these configurations.

\section{Conclusions}

For all configurations, the effect of Reynolds number was most evident at high yaw angles $(y>8 \mathrm{deg}$ and $y<-8 \mathrm{deg})$ where there was a significant reduction in drag at lower Reynolds numbers. However, this difference did not significantly affect the computation of the windaveraged drag coefficients at $55 \mathrm{mph}$ which uses data at lower yaw angles. As a result, the variation of Reynolds number for the baseline revealed an increase in the wind-averaged drag on the order of $1 \%$ for $\operatorname{Re} \leq 3$ million. The Reynolds-number sensitivity for side and roof extenders was more dramatic with an increase in wind-averaged drag of over $7 \%$ for $\operatorname{Re}=500,000$. Limited data for the boattail devices suggest that the Reynolds-number sensitivity is similar to that of the baseline. No Reynolds-number study was performed for the undercarriage flow barriers.

The PIV measurements in the tractor-trailer gap document significant crossflow velocities and recirculation regions at yaw angles near $10 \mathrm{deg}$. Baseline measurements with reduced drag exhibited significantly reduced crossflow and less coherent recirculation regions. Since the tractor side and roof 
extenders function to reduce the gap crossflow and increase the tractor back pressure, the addition of the extenders to the baseline reduced the measured drag at all yaw angles. This reduction was dramatic at high yaw angles (35\% at $10 \mathrm{deg})$ and minimal at low angles ( $2 \%$ at $0 \mathrm{deg})$. Of the four extender lengths tested $(0.3-0.6$ gap), the longest extenders were most effective yielding a $27 \%$ reduction in the windaveraged drag coefficient. Since extenders are a standard component of a modern tractor aero package, the effectiveness of the remaining dragreduction concepts were measured relative to this extender configuration. Note that no wall corrections were applied to the experimental measurements in order to facilitate comparison with CFD simulations. However, the influence of the tunnel walls was minimized by calculating the tunnel speed based on the static pressure at a location adjacent to the model.

Of the boat-tailing concepts (boattail plates and base flaps), the base flaps were found to be most effective. Base-flap angles from zero to 28 deg were studied resulting in an optimum angle of $20 \mathrm{deg}$. This result is higher than a previous low Reynoldsnumber study and may suggest some Reynoldsnumber sensitivity of the base-flap angle. The trailer belly box was the most effective trailer-

undercarriage concept with almost double the drag reduction of simple side skirts. The belly box and full skirt configurations were identical except for the lower-surface enclosure of the belly box. The drag of the full-skirt configuration, however, was increased while that of the belly-box configuration was significantly reduced. This result stresses the importance of eliminating cavity flows to minimize drag.

For more details and a list of references, please see the associated technical paper: AIAA-20042251.

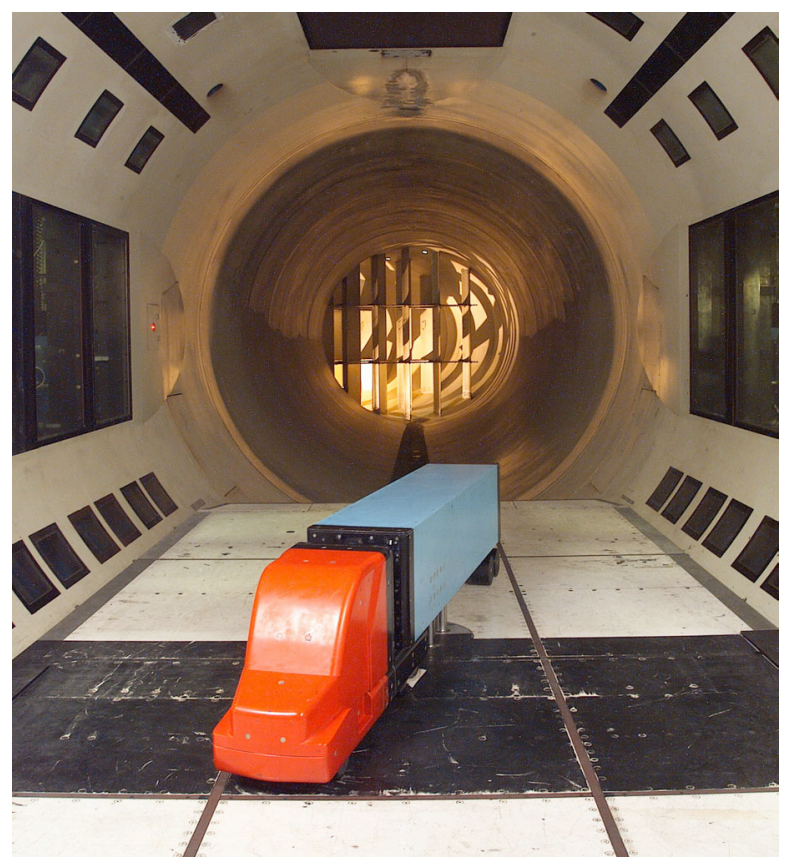

Figure 1: The Generic Conventional Model installed in the 12-Ft Pressure Wind Tunnel.

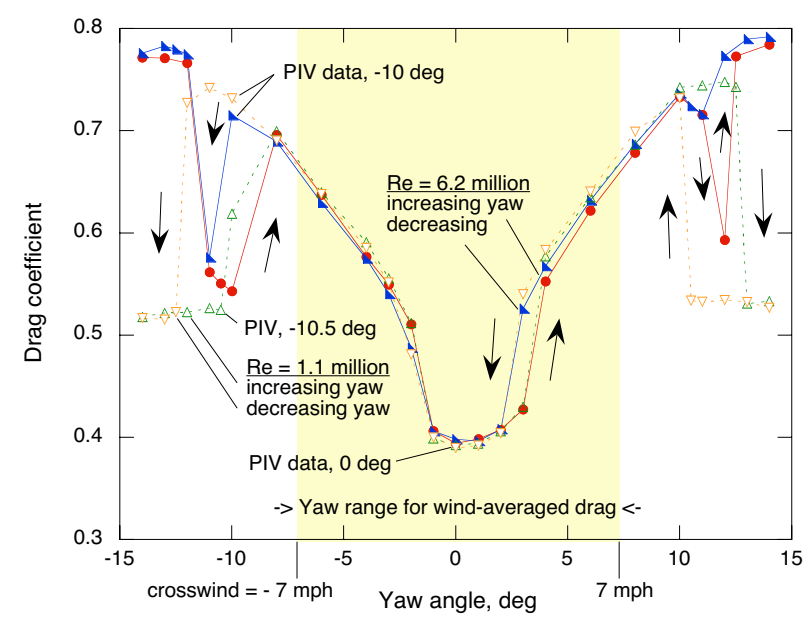

Figure 2: Baseline hysteresis of drag coefficient for two Reynolds numbers. 

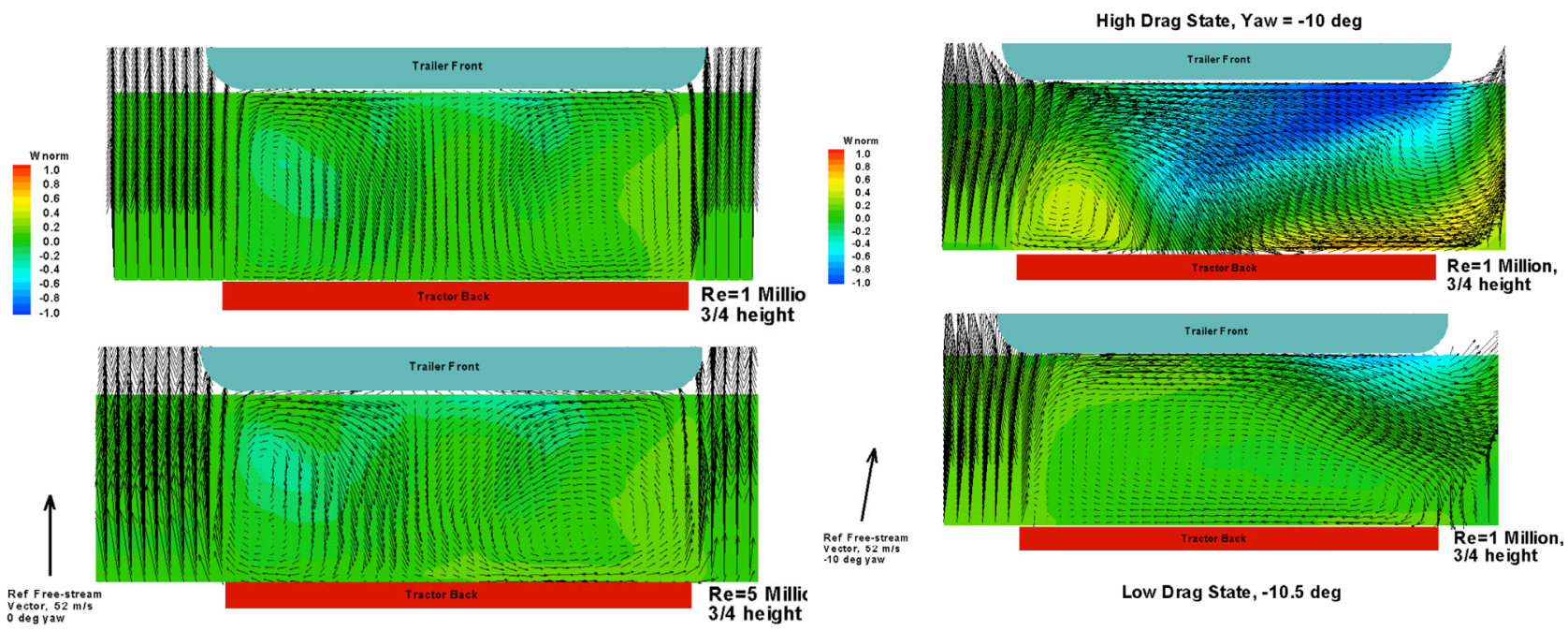

Figure 3: PIV measurements in the tractor-trailer gap at yaw $=0$ deg. Top view of horizontal PIV data plane at $0.75 \mathrm{~h}$.

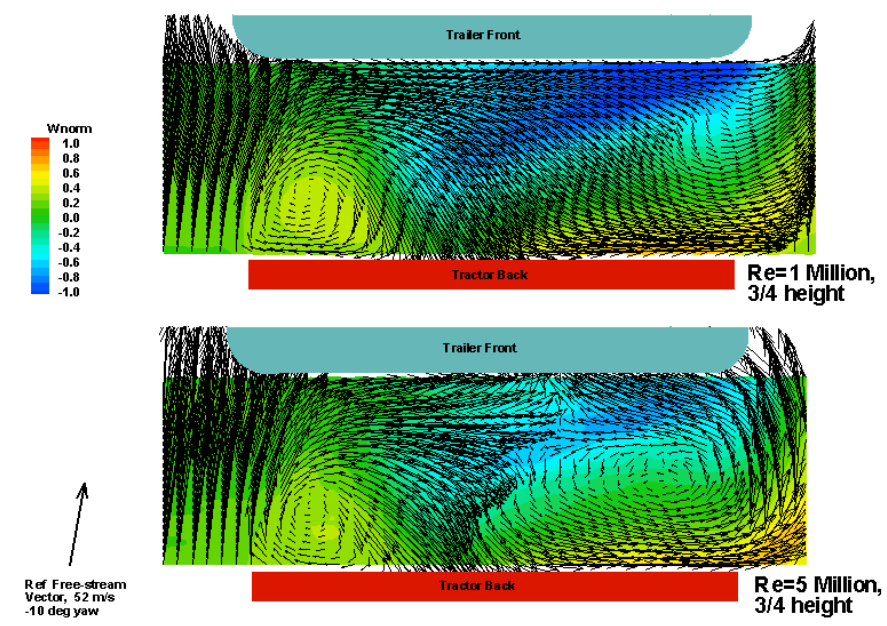

Figure 4: PIV measurements in the tractor-trailer gap at yaw $=-10 \mathrm{deg}$
Figure 5: PIV measurements in the tractor-trailer gap for high- and low-drag states near $-10 \mathrm{deg}$. Top view of horizontal PIV data plane at $0.75 \mathrm{~h}$.

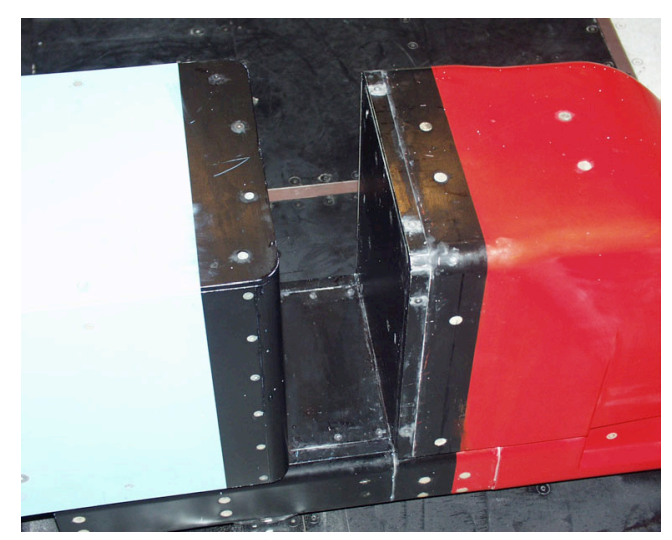

a) Baseline Configuration

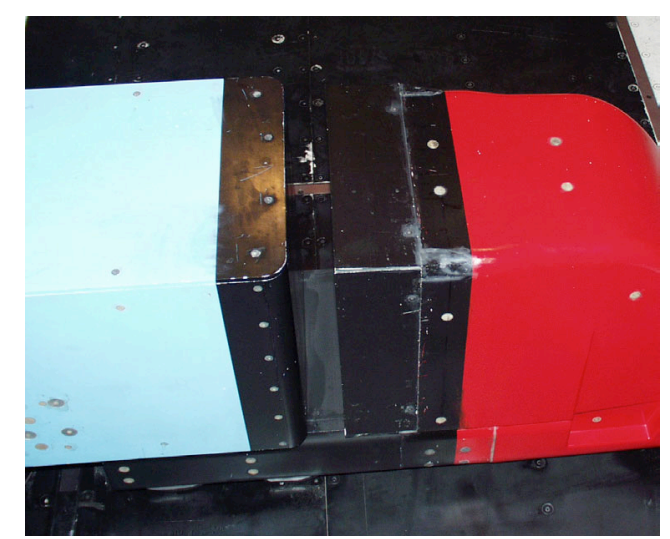

b) Side and Roof Extenders $(0.6 \mathrm{~g})$

Figure 6: Close-up of tractor-trailer gap with and without side and roof extenders. 


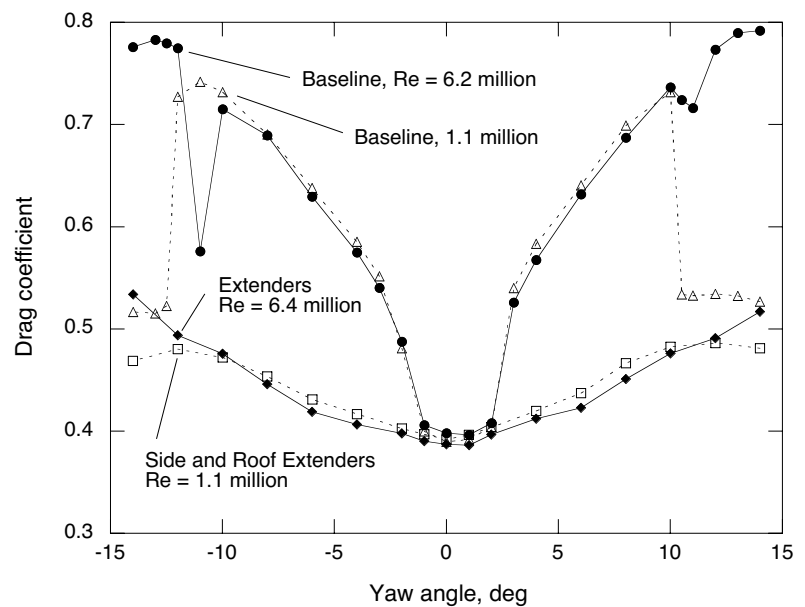

Figure 7: Effect of tractor side and roof extenders $(0.6 \mathrm{~g})$ on total drag coefficient.

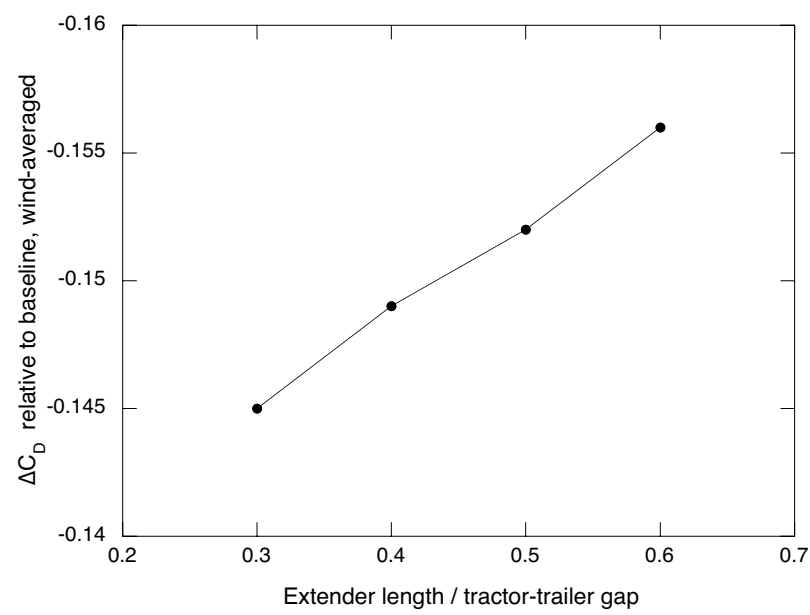

Figure 8: Wind-averaged drag reduction due to side and roof extenders at $\mathrm{Re}=6$ million.

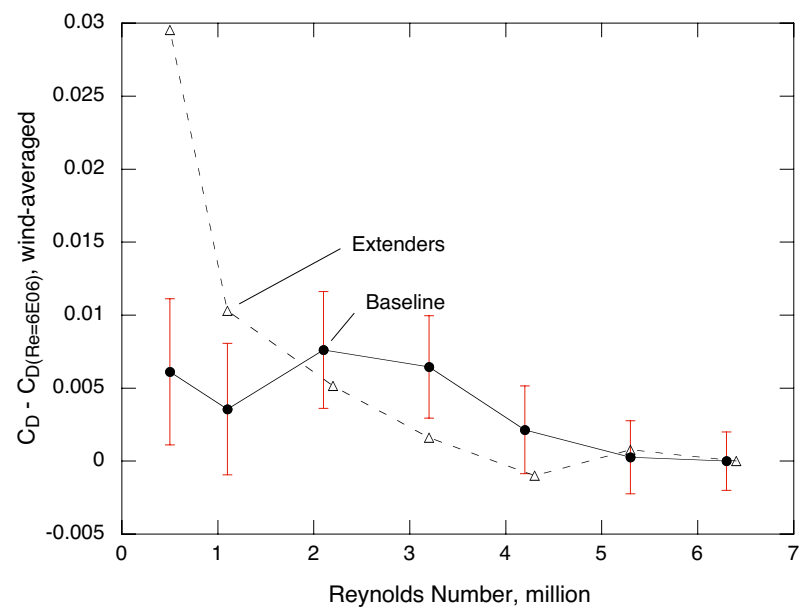

Figure 9: Reynolds-number sensitivity of windaveraged drag for baseline and side extenders.

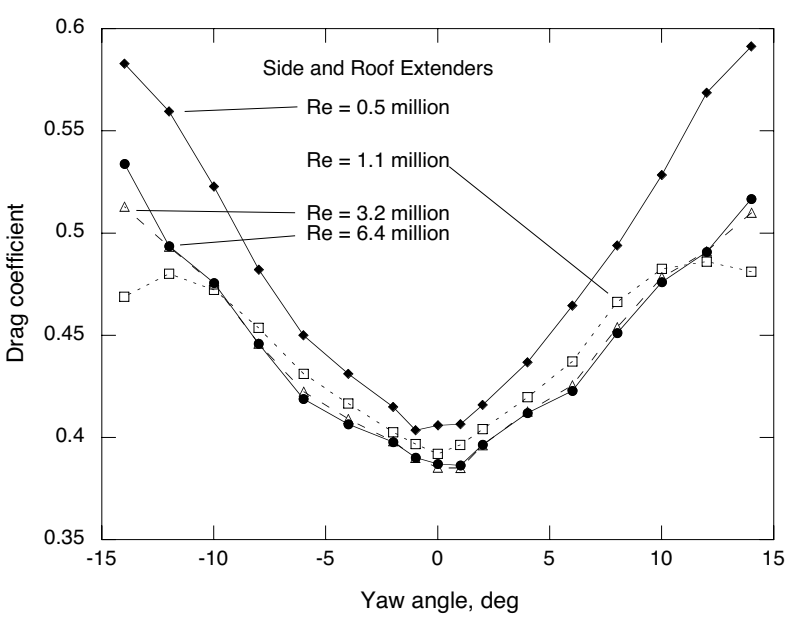

Figure 10: Reynolds-number sensitivity of side and roof extenders on drag curves.

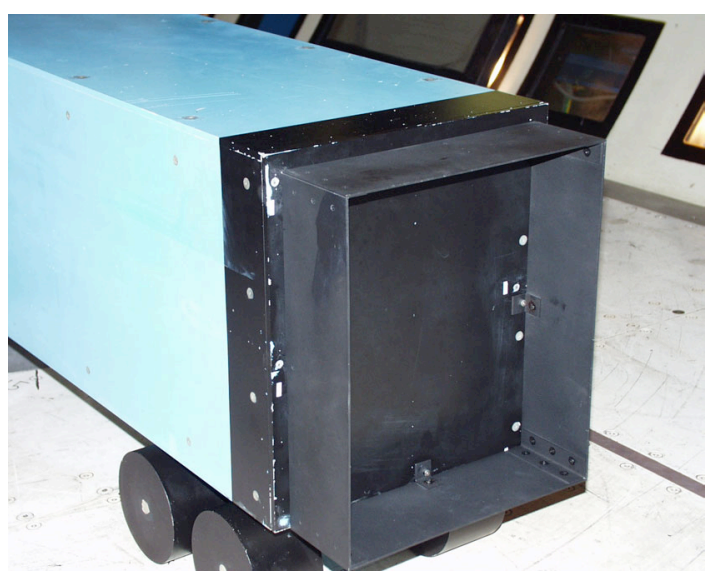

Figure 11: Aerodynamic boattail plates installed on the base of the trailer.

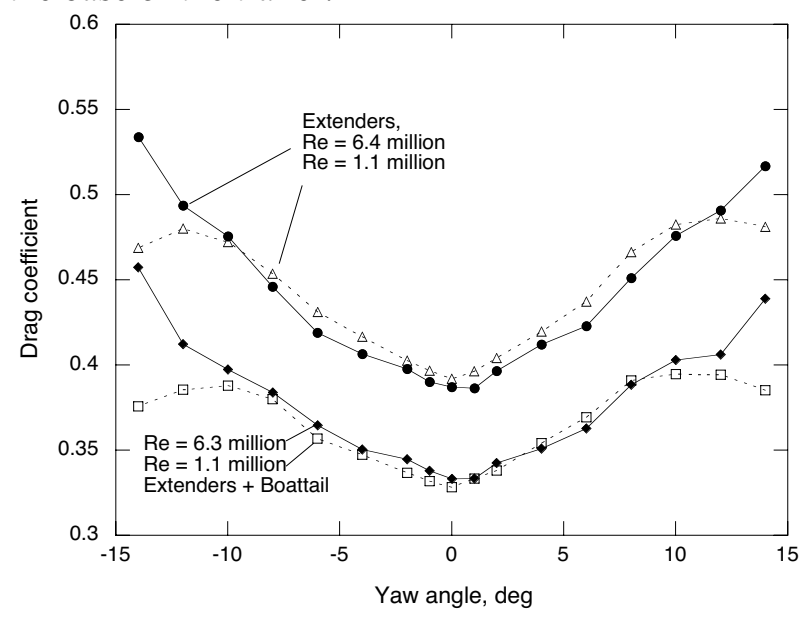

Figure 12: Effect of boattail plates on total drag coefficient. 


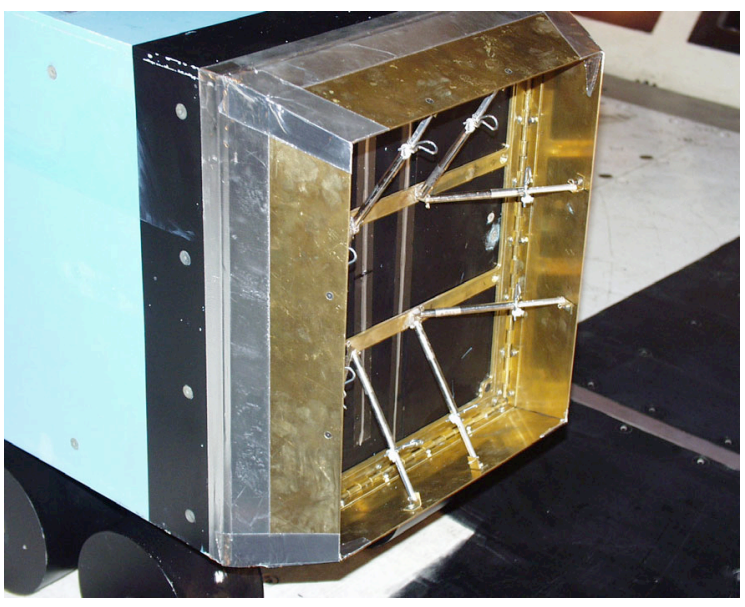

Figure 13: Base flaps installed on trailer base (flap angle $=20 \mathrm{deg}$ ).

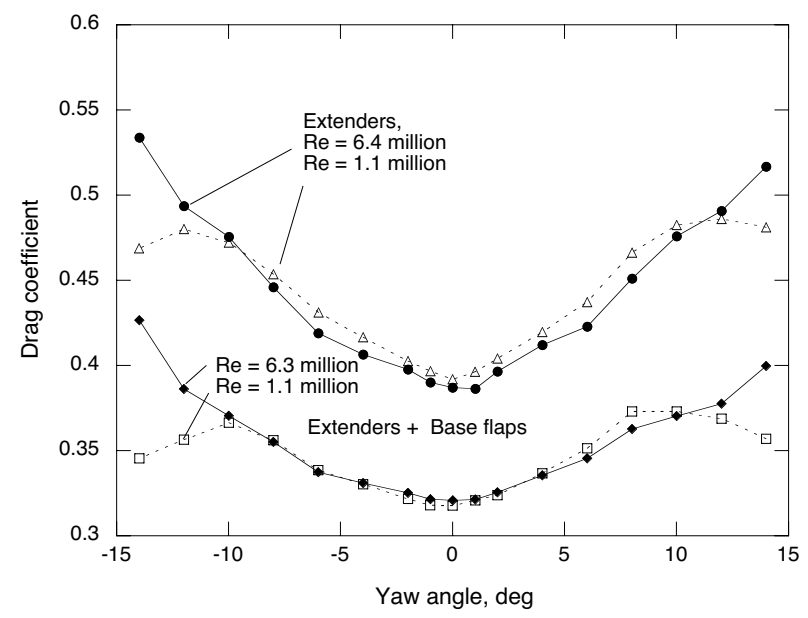

Figure 14: Effect of 16-deg base flaps of total drag coefficient.

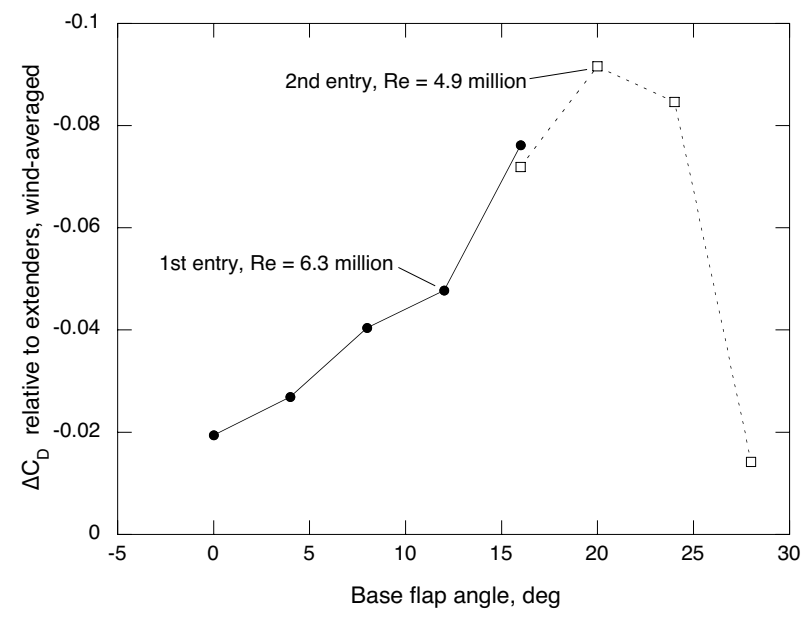

Figure 15: Effect of base-flap angle on windaveraged drag reduction.

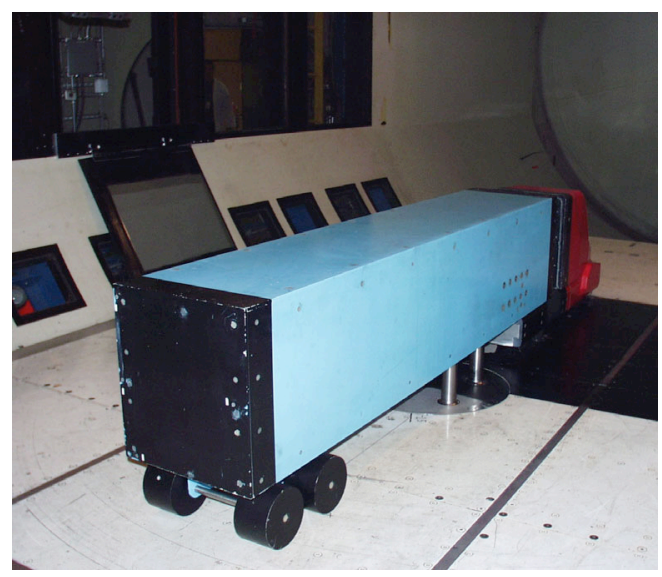

a) Baseline (no belly box)

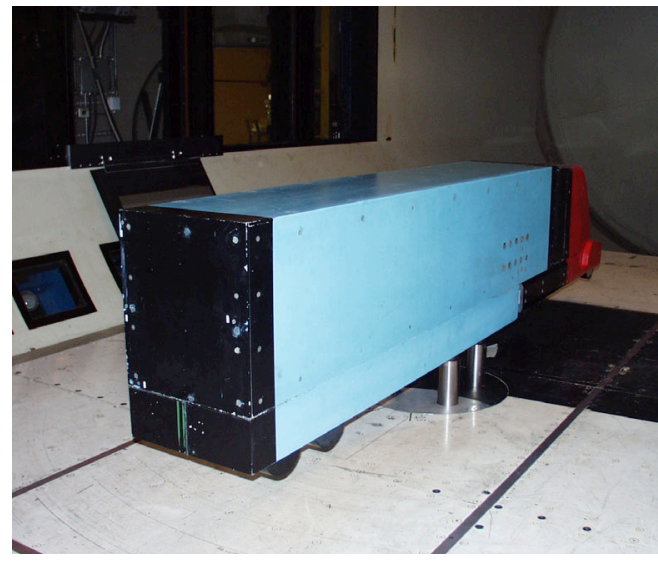

b) Trailer with belly box

Figure 16: Rear view of trailer with and without belly box.

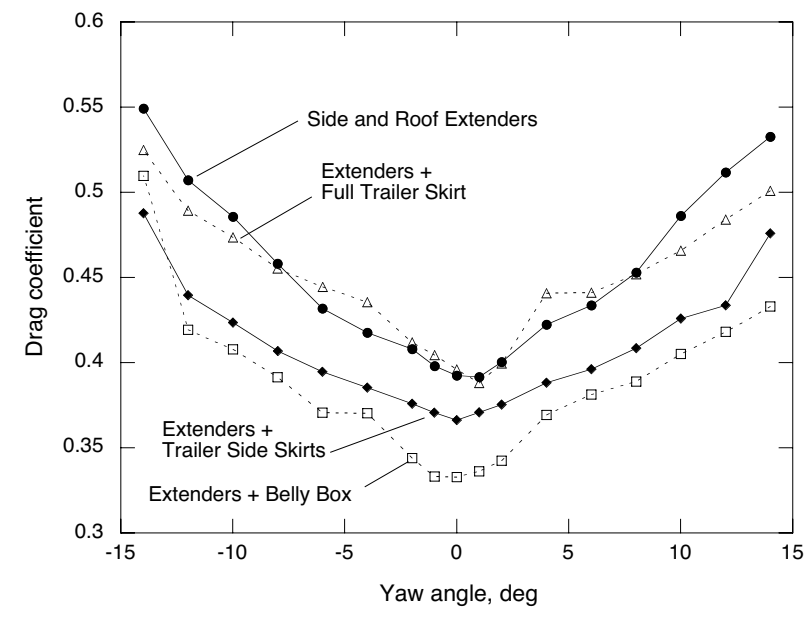

Figure 17: Effect of trailer belly box and skirts on total drag coefficient $(\operatorname{Re}=6.3$ million $)$. 


\section{B. An Experimental Study of Aerodynamic Drag of Empty and Full Coal Cars}

\section{Objective}

- To investigate the additional aerodynamic drag experienced by empty coal cars relative to cars full of coal and methods to reduce the empty-car drag

\section{Approach}

- Small-scale wind-tunnel tests of empty and full coal cars and associated drag-reduction devices. $1: 87^{\text {th }}$ scale models were used - drag measured using a miniature 2-pound linear load cell

\section{Accomplishments}

- The drag penalty of empty coal cars relative to full cars varies from $35 \%$ to $42 \%$ depending on the direction of the prevailing wind $\left(0\right.$ to $\left.10^{\circ}\right)$

- Initial tests of vertical plates in the empty car showed a $\sim 50 \%$ reduction in the drag penalty for empty cars

\section{Future Direction}

- Additional drag-reduction devices will be examined in collaboration with a coal car manufacturer and Lawrence Livermore National Laboratory researchers

As part of a DOE-sponsored study, the aerodynamic drag of $1 / 87^{\text {th }}$-scale coal cars was measured in a wind tunnel. The goal of the experiment was to measure the difference in drag between full and empty cars so as to determine if drag mitigation efforts are warranted.

The measurements were made in the NASAAmes 15 x 15 Inch Low-Speed Wind Tunnel. This is an open-circuit, suction-type tunnel with at square test section measuring 60 inches in length. Five coal cars were mounted on scale train track (Fig 1) with the middle car connected to the upwind car by a 2-lb load cell (Fig 2) and disconnected from the downwind car. All cars except the middle car were glued to the track to prevent motion along the track. This configuration was tested at a freestream velocity of $65 \mathrm{~m} / \mathrm{s}(145 \mathrm{mph})$ with and without simulated coal. The tunnel speed was chosen to maximize the measured drag and minimize measurement uncertainty. The five-car combination was tested at yaw angles of zero and $10 \mathrm{deg}$ to determine the effects of crosswind. The blockage of the coal cars at zero yaw was $0.9 \%$.

The small- and full-scale Reynolds numbers of the coal cars (assuming full-scale velocity of 60 mph) are 0.16 and 5.8 million, respectively. Due to the nature of bluff-body flow fields, this difference in Reynolds number is not expected to significantly affect the experimental results. The drag coefficient for each configuration (Table 1) was calculated based on the square root of the zero-yaw frontal area. The uncertainty based on measurement resolution and repeatability was 1.2 $-2.5 \%$ depending on the magnitude of the drag measurement. As indicated below, the drag on the full coal car was significantly less than that of the empty car (32\%). This difference was even greater $(42 \%)$ for the $10-$ deg yaw condition in which the drag was significantly increased relative to the zero-yaw case. These results suggest that significant fuel savings could be obtained by reducing the drag of the empty coal cars.

Vertical dividers were installed in order to manipulate the flow inside the cars with the goal of making the external flow more like it is when the car is loaded with coal. The dividers had a significant effect on the drag. The single divider reduced the empty-car drag by $15 \%$ at zero yaw. The three dividers reduced the empty-car drag by $21 \%$. More work is needed to identify the overall best approach to drag reduction. This effort will 
be considerably helped by the concurrent CFD being performed at Lawrence Livermore National
Laboratory.

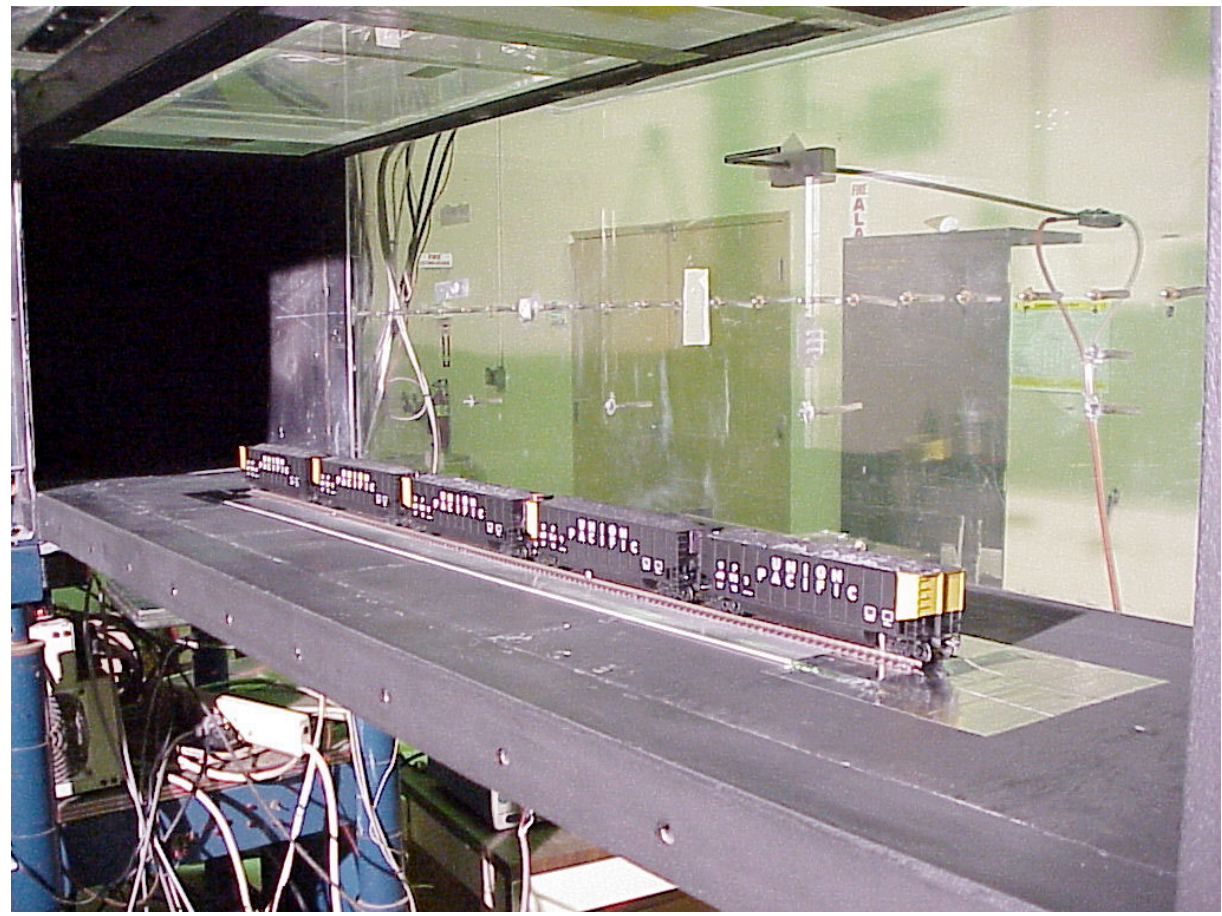

Fig. 1 Five coal cars mounted in wind-tunnel test section. Pitot-static probe used for tunnel speed measurement is visible above first car near the top of the photo.

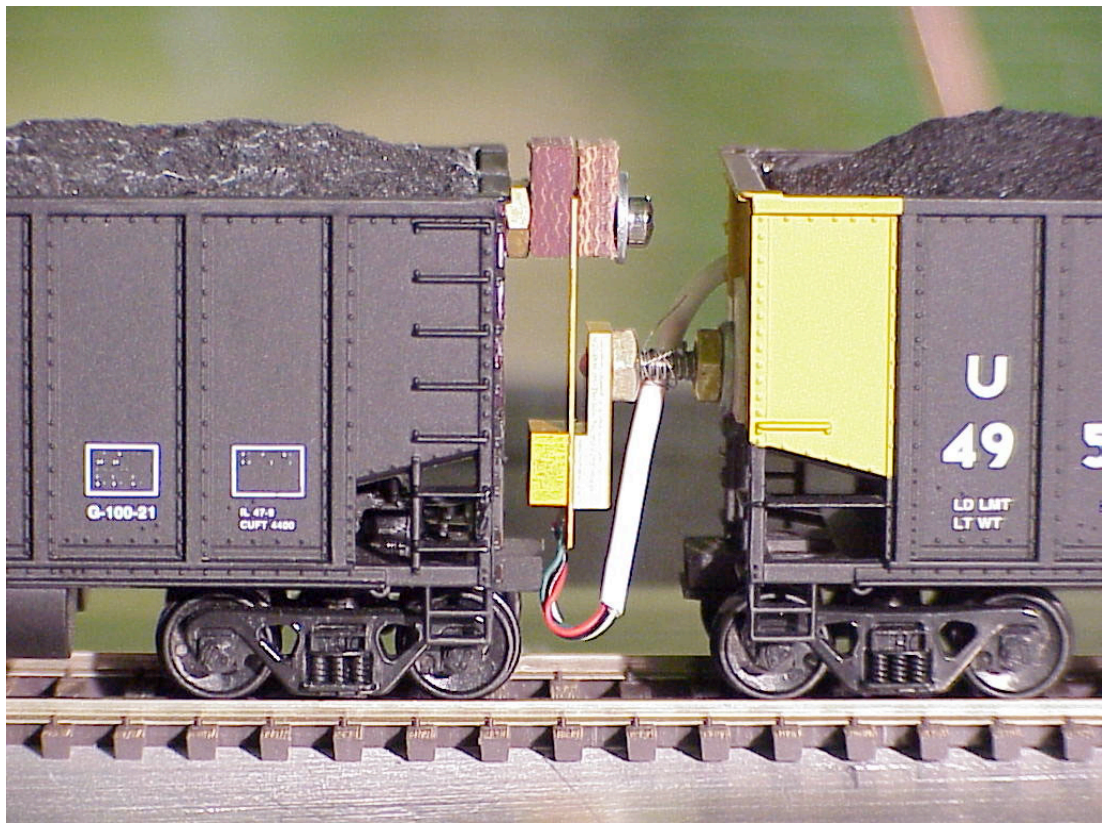

Fig. 2 Load cell connecting metric car (at left) to upwind car (at right). The load cell is the thin vertical strip at the center of the photo. Instrumentation wiring enters upwind car in the white cable. Simulated coal is visible in both cars. 


\begin{tabular}{|l|l|l|l|}
\hline Yaw, deg & Cd empty & Cd full & \%diff \\
\hline 0 & 0.315 & 0.216 & 31 \\
\hline 10 & 0.519 & 0.300 & 42 \\
\hline
\end{tabular}

Table 1 Drag coefficients for full and empty coal cars at 0 and 10 deg yaw (empty car is reference at each yaw angle).
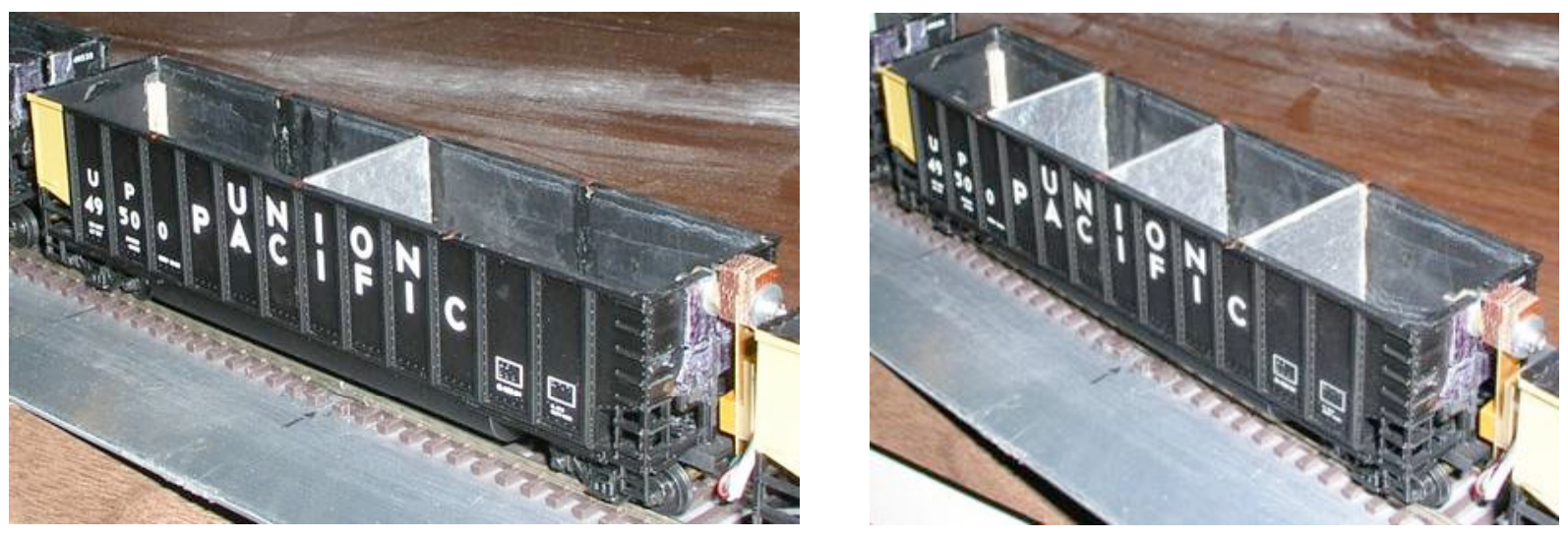

Fig. 3 Vertical plates installed in model cars. Single and three evenly-spaced veritical dividers. 


\title{
APPENDIX B \\ Experimental Measurement of the Flow-field of Heavy Trucks
}

\author{
Principal Investigator: Fred Browand \\ Aerospace \& Mechanical Engineering, University of Southern California \\ RRB 203, Los Angeles CA 90089-1191 \\ (213) 740-5359; fax: (213)740-7774; e-mail: browand@spock.usc.edu \\ Technology Development Area Specialist: Sidney Diamond \\ (202) 586-8032; fax: (202) 586-1600; e-mail: sid.diamond@ee.doe.gov \\ Technical Program Manager: Jules Routbort \\ (630)252-5065; fax: (630)252-4289; email: routbort@anl.gov
}

Contractor: National Energy Technology Laboratory, Pittsburgh, PA 15236-0940

Contract No.: DE-AC03-98EE50512

\section{Objective}

- Improve the performance of heavy trucks by reducing aerodynamic drag and by increasing safety.

\section{Approach}

Identify areas where improved use of aerodynamic design could decrease truck drag and consequently improve fuel economy. These include:

- Gap between tractor and trailer - We have identified the importance of cross-gap flow as a source of drag increase.

- Trailer base - We have performed wind tunnel testing to evaluate the performance of various flapped devices to close the trailer- base wake more efficiently.

\section{Accomplishments}

\section{Minimizing drag due to cross-gap flow}

- Wind tunnel flow field studies document the appearance of violent cross-gap flows under certain conditions.

- The cross-gap flow has been studied by means of a special analysis procedure entitled Proper Orthogonal Decomposition.

- Suggestions are made to minimize this unwanted cross-gap flow by means of a simple splitter plate attached to the front of the trailer and partially covering the gap.

\section{Field measurement of fuel saving using a trailer-base add-on}

- Field tests directly measure fuel consumption of a Class- 8 tractor and trailer with and without flaps attached to the sides and top of the trailer. The most promising flap geometry for field test was chosen on the basis of wind tunnel tests.

- The field test documents fuel saving of about $4 \%$; to our knowledge, this saving is the best that has been achieved for a simple, passive, trailer-base add-on. 


\section{Future Direction}

- Initiate a program and a new testing apparatus to study wheel/tire splash and spray.

This report describes the progress we have made on two separate aerodynamic problems (I) describing the sensitivity of the drag to the geometry of the gap between tractor and trailer, and (II) providing field test results of high quality to demonstrate the fuel saving to be realized for flaps attached to the base of a trailer.

\section{Minimizing drag due to cross-gap flow}

Certain aspects of the tractor-trailer gap flowfield have been described in a paper (entitled:

"Flow Structure in the Gap Between Two Bluff-Bodies", D. Arcas, F. Browand \& M. Hammache, AIAA Paper No. 2004-2250) prepared for the AIAA meeting in Portland June 28-July 1, 2004. The gap data is presented in the mathematical format of a Proper Orthogonal
Decomposition, which will facilitate comparisons between the experimental results and the ongoing numerical computations at LLNL.

This work and earlier studies describing the gap flow (in a paper entitled: "On the Aerodynamics of Tractor-Trailers," M. Hammache \& F. Browand, Proceedings of the UEF Conference on: The Aerodynamics of Heavy Vehicles:Trucks, Buses and Trains, Lecture Notes in Applied and Computational Mechanics Springer-Verlag, Heidelberg, September, 2004) have provided a clear and quantitatively correct picture of the flow field within the gap for a variety of flow conditions. An example of the gap flow for an angle of yaw of 6 degrees is shown in Figure 1.
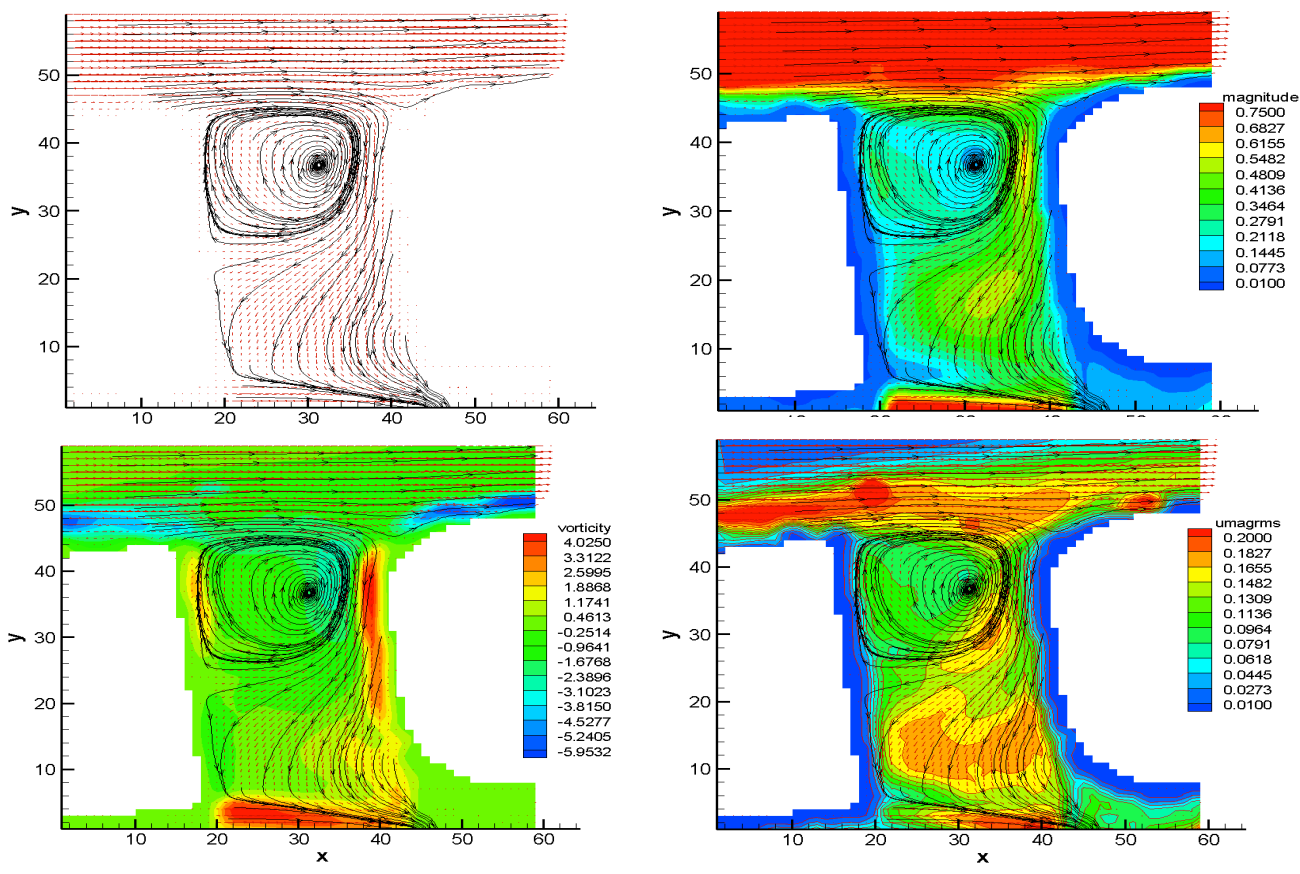

Figure 1. Wind tunnel model of simple tractor-trailer gap flow at a gap of _trailer width. The cab outline to the left and the trailer outline to the right are yawed 6 degrees to simulated a side-wind.

The velocity field within the gap is determined from Digital Particle Image Velocimetry (DPIV) measurements. Accurate mean-flow streamlines are superimposed upon several field quantities plotted in color in the upper left of Figure 1. On the upper right of Figure 1, is the mean velocity magnitude; to the lower left is mean, or average, vorticity; to the lower right is the root-meansquare amplitude of the unsteady fluctuation. The flow crossing the gap can be seen to be quite 
large-especially in the vicinity of the front of the trailer. The flow picture suggests that a short fin, or plate, protruding from the front of the trailer might serve to substantially or entirely disrupt the cross-gap flow.

\section{Splitter-Plate Gap Stabilization}

A follow-on experiment, performed by Eric Liu at USC shows the effect upon trailer drag of a splitter plate of various lengths placed at the nose of the trailer in the center-plane. The geometry is shown in Figure 2 and photographs of the model in the USC wind tunnel are shown in Figures 3 and 4 . The gap, $\mathrm{G}$, is normalized by the square root of the cross- sectional area, $\sqrt{\mathrm{A}}=181 \mathrm{~mm}$.

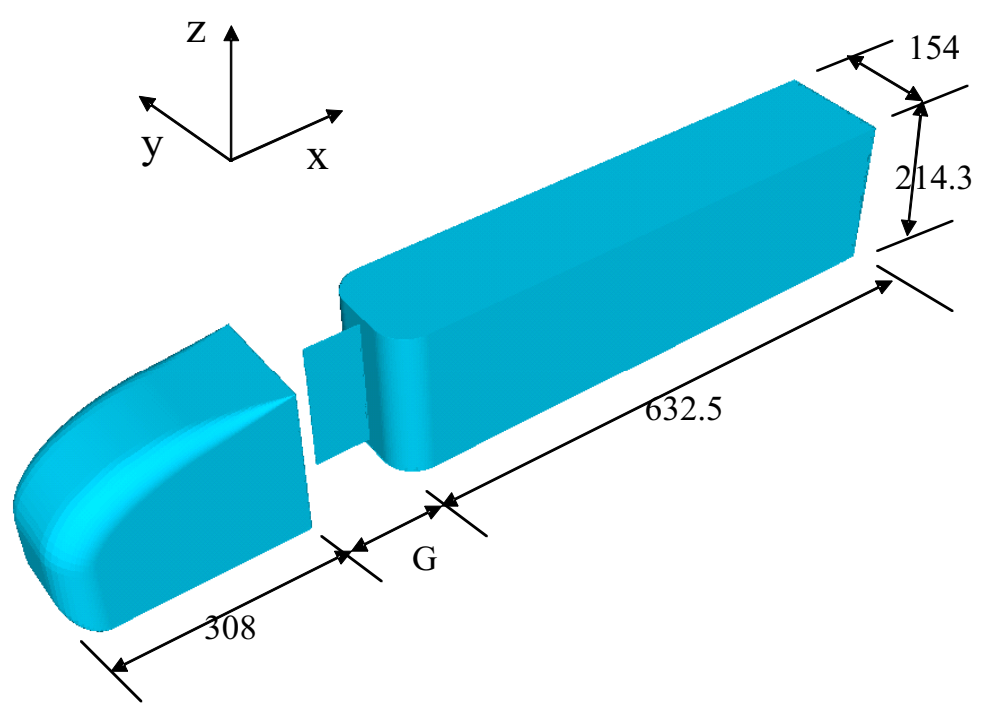

Figure 2. Geometry of wind tunnel model (dimensions in $\mathrm{mm}$ ).

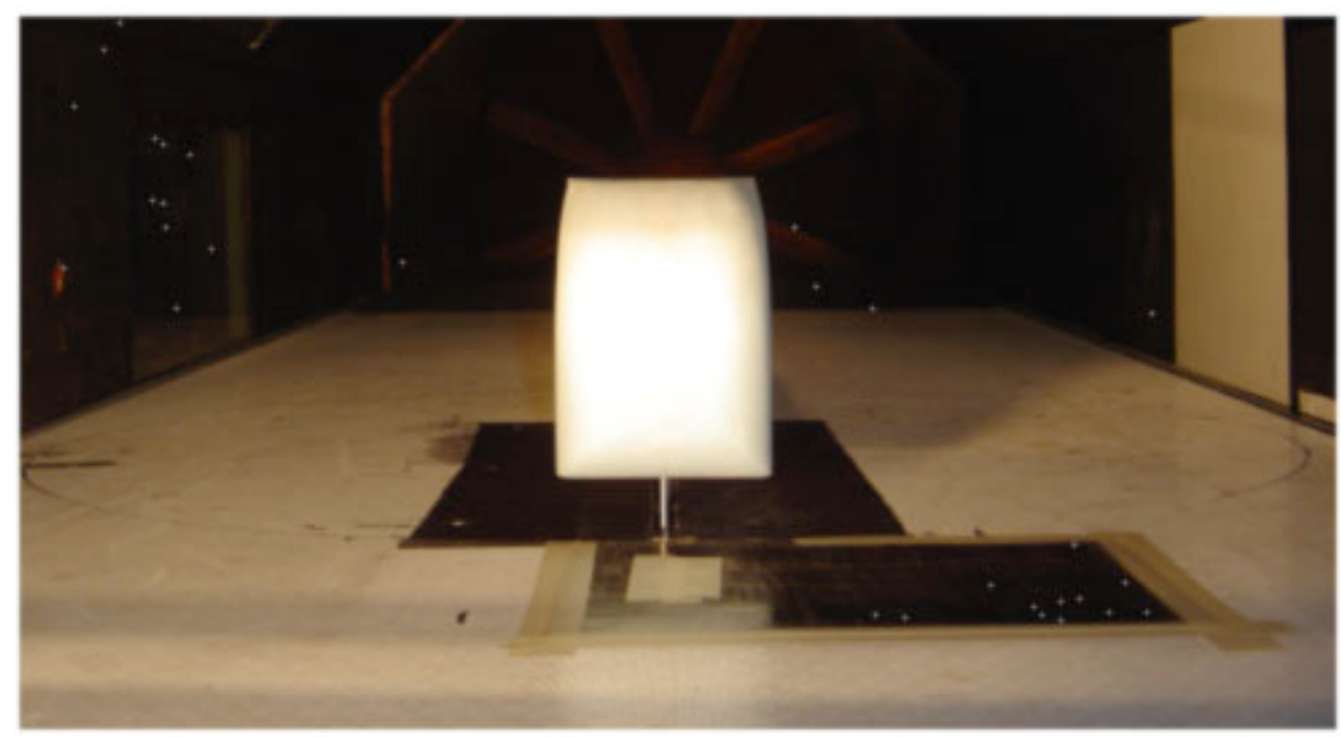

Figure 3. Head-on view of model in wind tunnel. 


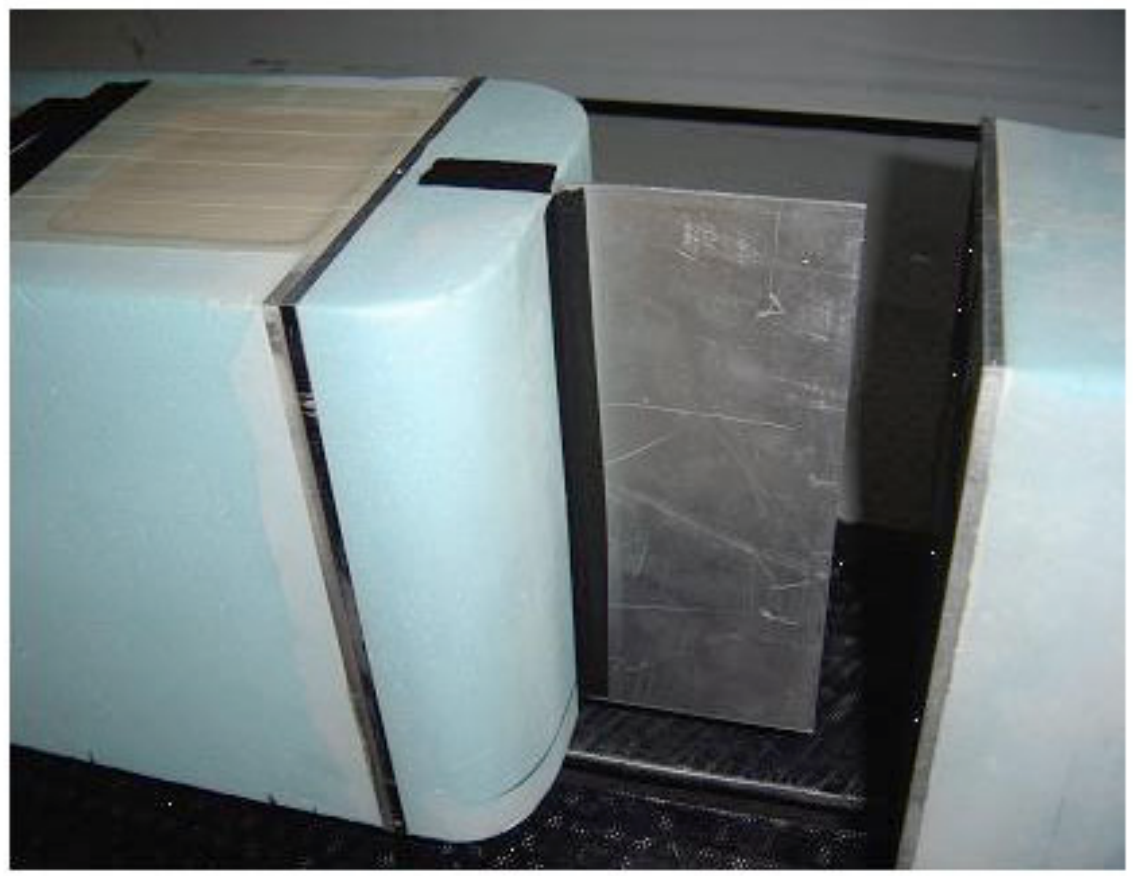

Figure 4. Detail of splitter plate installation.

The results of the test are given in figures 5 showing drag coefficient for the trailer as a function of yaw angle for various lengths of splitter plate. There is little change in drag at small yaw angles, but as yaw angle increases the splitter plat becomes more effective. All three splitter plate lengths have about the same

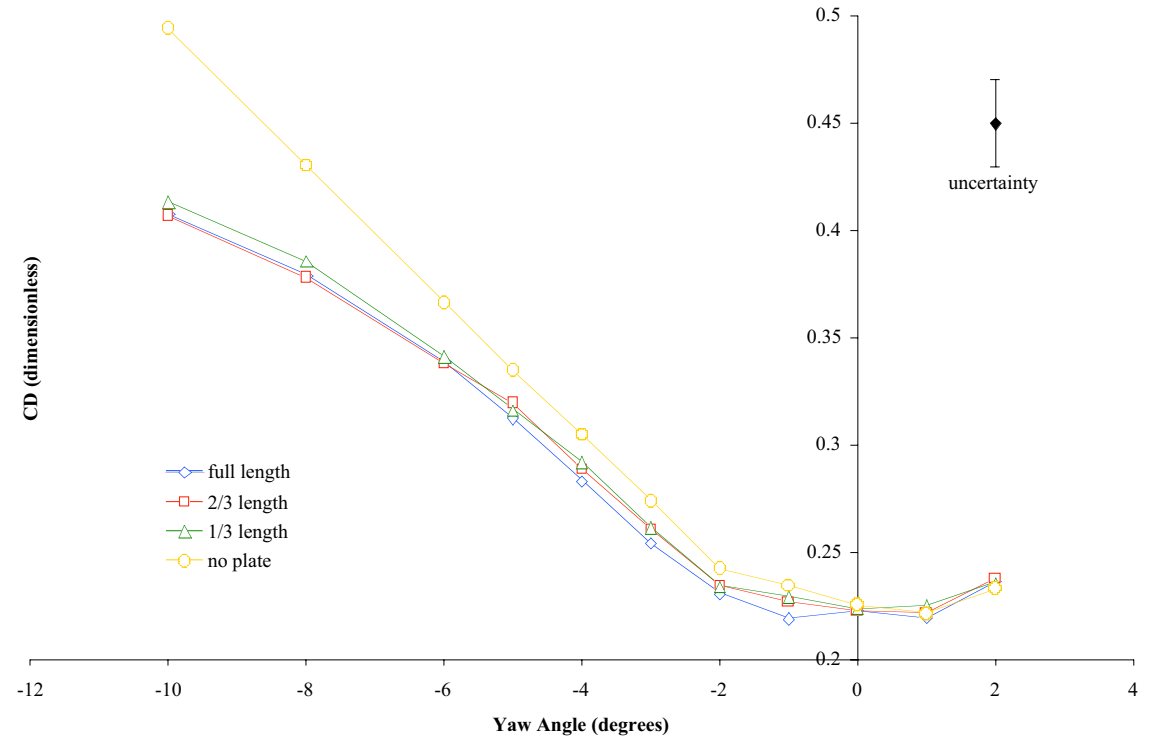

Figure 5. $C_{D}$ for three splitter plate lengths versus yaw angle at $G=0.5(G=$ gap $/ \sqrt{ } A)$.

performance at this gap length, but at larger gaps (not shown) the longer plates show less improvement and can actually lead to drag increases. For this reason, the shorter plate would 
seem to be the desirable choice. Figure 6 gives drag for the $1 / 3$ gap length splitter plate for three separate gap lengths with yaw angle as a parameter. The results are quite consistent in producing a drag saving. We suggest that such simple fin geometries be given wind tunnel testing at full-scale, or be subjected to over-the-road testing where operation can be evaluated under actual field conditions.

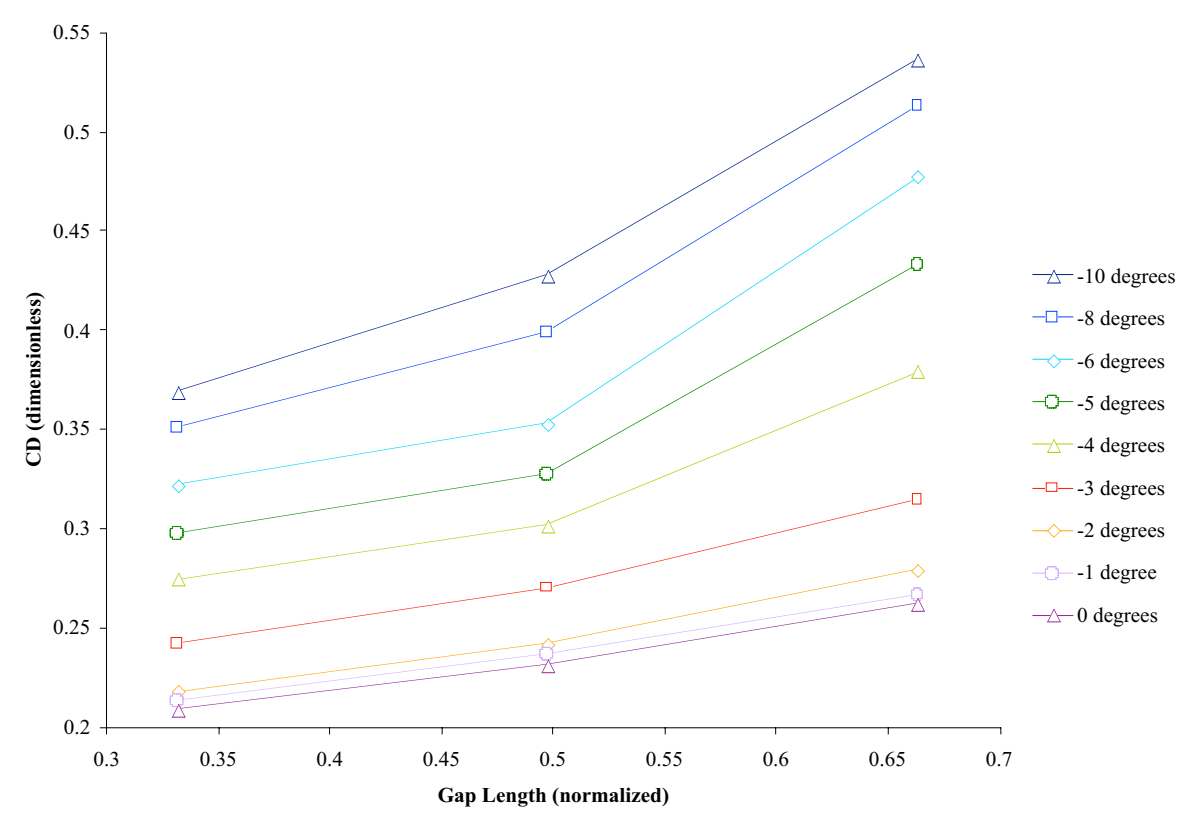

Figure $6 . C_{D}$ for $1 / 3$ gap length splitter plate as a function of normalized gap length.

\section{Field measurement of fuel saving using a trailer-base add-on}

A very successful field test was performed at the NASA Crows Landing Flight Facility on May 17-22 to evaluate the anticipated fuel saving associated with base-flap add-ons. USC organized and participated in the tests. The base flaps and the trailer were provided by Norcan Aluminum Incorporated, and the tests were conducted with trucks and personnel from California PATH at UC Berkeley. The data acquired was of high quality for a field test. It showed an optimum fuel consumption saving of about $4 \%$ at an optimum flap angle of about $13^{\circ}$--although the entire flapangle range from $10^{\circ}-16^{\circ}$ would provide nearly the same saving. The modification to the trailer base is simple and relatively inexpensive to implement. The test results, and the unique experimental procedure for the tests, are documented for presentation at the SAE World Congress, April 2005 (paper \# 05-B83, "Fuel Savings by Means of Flaps Attached to the Base of a Trailer: Field Test Results", F. Browand, C. Radovich \& M. Boivin).

\section{The site at Crows Landing}

The present tests are performed at the NASA Crows Landing Flight Facility at the northern end of the San Joaquin valley. The main runway is approximately 2400 meters in length, and is oriented roughly north-south, as shown in Figure 7.

\section{Truck and trailer}

A single Freightliner 2001 Century Class truck is used for the tests. The truck is powered by Cummins N14 Celect engine developing a maximum of $350 \mathrm{HP}$. The truck has an automatic transmission, Allison HD 4060 (six forward gears), and a rear axle ratio of 4.63. In operation, the truck executes multiple runs up and down the runway. A run consists of an acceleration phase to a predetermined speed, a uniform speed phase, and a deceleration phase. First, an achievable speed trajectory is established for the truck on the runway. This desired speed trajectory is then programmed into an on-board computer that controls the truck, and insures that all runs are executed in identical fashion. 


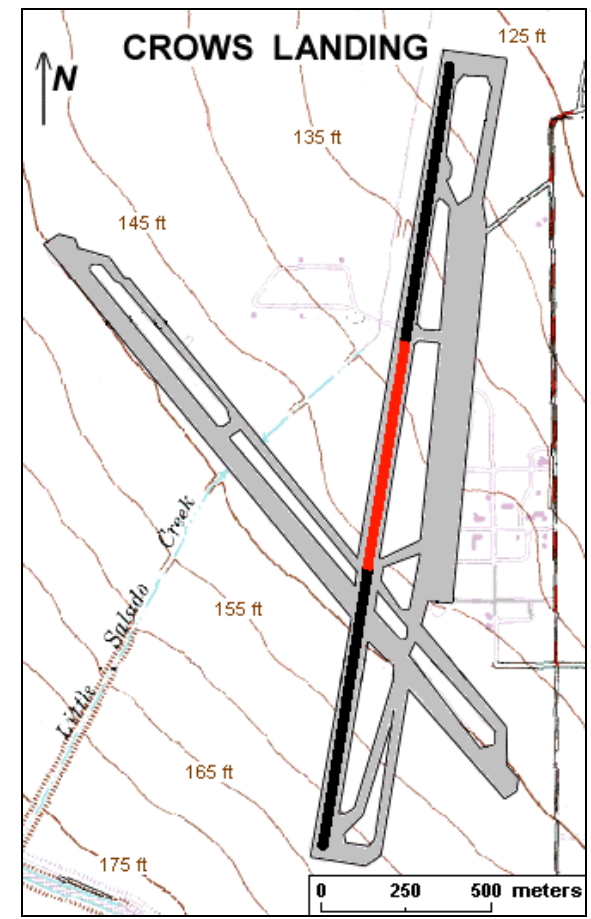

Figure 7. Plan view of the NASA Crows Landing Flight Facility. Red bar marks measurement interval on runway.

\section{The base flaps}

Base flaps are attached to the sides and top of the rear of the trailer. The flaps are constructed from a fiberglass-epoxy-resin material and are one-quarter of the base width in length (about 61 $\mathrm{cm}$, or 2 feet). Figure 8 presents several of the flaps used for the test. The side-flaps swing on piano hinges that are bolted directly to the rear side-edges of the trailer to produce a sealed joint. This installation detail is dictated by the need to quickly remove the flaps for the "no-flaps" control runs.

The flap angle is adjusted by means of two aluminum supports. Holes are pre-drilled to allow the five flap angles to be set quickly. In commercial application, the flaps are attached along the rear door hinge lines, so that no gap appears at the joint between the flap and the side of the trailer. Also in commercial application, the flaps are constrained only by a short length of cable attached to the rear door. Higher pressures on the trailer base and on the inside of the flap, compared to the stream side of the flap, are sufficient to keep the flap extended at highway speeds.
The flap at the top of the rear door is split so the doors can be opened. The two flap-halves are mounted by means of hinges and are kept in place by means of adjustable turnbuckles, Figure 8 upper right. The split in the top flap is sealed with duct tape. The "no-flaps" control is obtained by completely removing flaps from the sides, and taping the top flaps against the rear door.

\section{Test procedure}

A typical run sequence starts at a fixed point at one end of the runway. Computer control is initiated, data acquisition begins, and the truck accelerates. When the programmed acceleration ramp terminates, the truck continues along the runway at the preset cruise speed of 26.8 meters/second (60.0 mph). Distance along the track is determined by integration of the forward speed. At a pre-determined distance, the braking sequence is initiated, and the truck slows to a stop at the far end of the runway. Data acquisition stops, and the run file is logged in the computer. The truck is turned, and made ready for the return from a second fixed point on the track. Typically a run and the return run are accomplished within 
about 6 minutes. A total of 16 runs, or 8 run-pairs are accumulated for each flap angle setting (about 45 minutes of test time), and constitute one data set. When a data set is completed the truck is returned to the garage area, and a new flap angle is positioned. Setting a new flap angle usually takes 20-30 minutes. Four flap angles - corresponding to four data sets - are accumulated each day during the 6:00-10:30 AM period when wind and temperature are most favorable. The total data base over five testing days, May 17-21, consists of 304 runs (152 run-pairs) - or 19 data sets - at the flap angles 10,13, 16, 19 and 22 degrees, as well as the no-flaps condition that serves as the control.

A total of ten variables are recorded for each data set. The variables include time, integrated distance, engine torque, engine rpm, vehicle speed and instantaneous fuel consumption (broadcast fuel rate). All of these signals are commonly available on the J1939 bus.
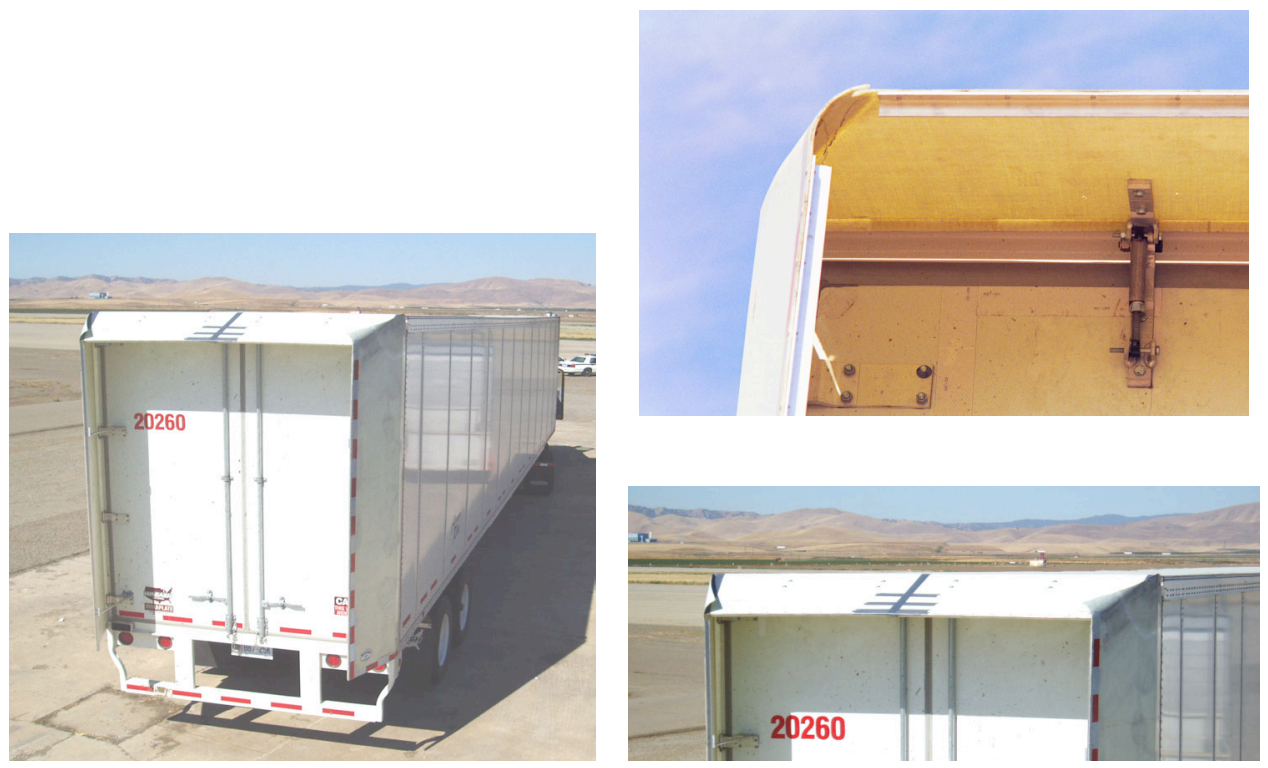

Figure 8. Views of flap installation.

\section{Results}

Plotted as the solid symbols in Figure 9 are the averaged results - excluding the four data sets showing the least internal consistency (data set rms $>1.5 \%$ ). In addition, the dashed bars give the estimated $99 \%$ confidence interval for each flap angle. The confidence interval is determined from the standard deviation estimate at each flap angle. A $99 \%$ confidence interval suggests that if the tests are repeated under the same conditions, the averaged values will lay within the bounds of the confidence bars $99 \%$ of the time. While our repeated sampling over the week-long period does not alter the variability inherent in the data, repeated sampling does provide a much more accurate estimate of the mean (averaged) values.

Also shown are the two other data reduction choices - triangles indicate the result when all the data is utilized, and squares indicate the result when only runs at low wind intensity are kept. These three results are not substantially different - they all lie within the $99 \%$ confidence bounds. Expressed as a fraction of the fuel consumption without flaps, the averaged values are accurate to about $\pm 0.6 \%$. 


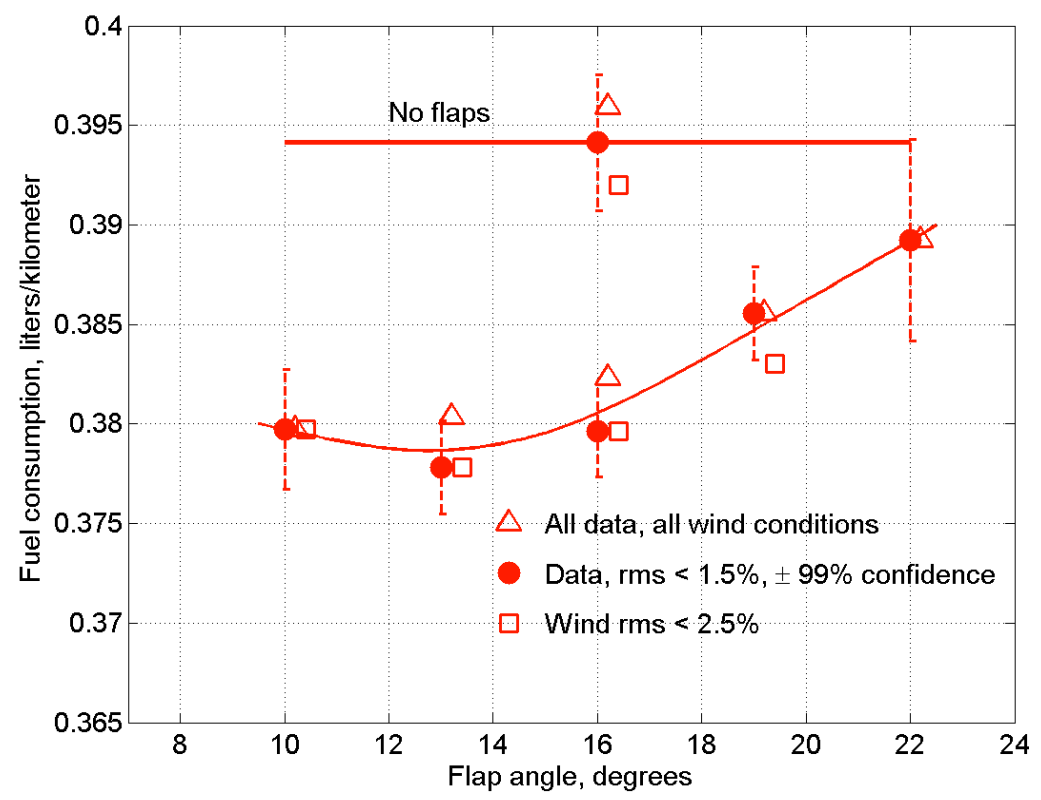

Figure 9. Fuel consumption savings versus flap angle, various data reduction strategies.

\section{Implications for fleet operation}

There is minimum fuel consumption at a flap angle of about 13 degrees, but the minimum is broad - the fuel consumed at 10 degrees and 16 degrees is only marginally greater. It would be advantageous from an operations standpoint to have such a broad minimum.
The saving in fuel consumption arising from the use of base flaps at a $13^{\circ}$ flap angle is 1.63 liters $/ 100 \mathrm{~km}$, or in gallons and miles, 0.693 gal/100 miles. A dollar values can be placed on the accumulated saving by assuming a price for fuel. Figure 10 shows the results for a fuel price of $\$ 2.20$ /gal for distances of 50,000 and 100,000 highway miles traveled.

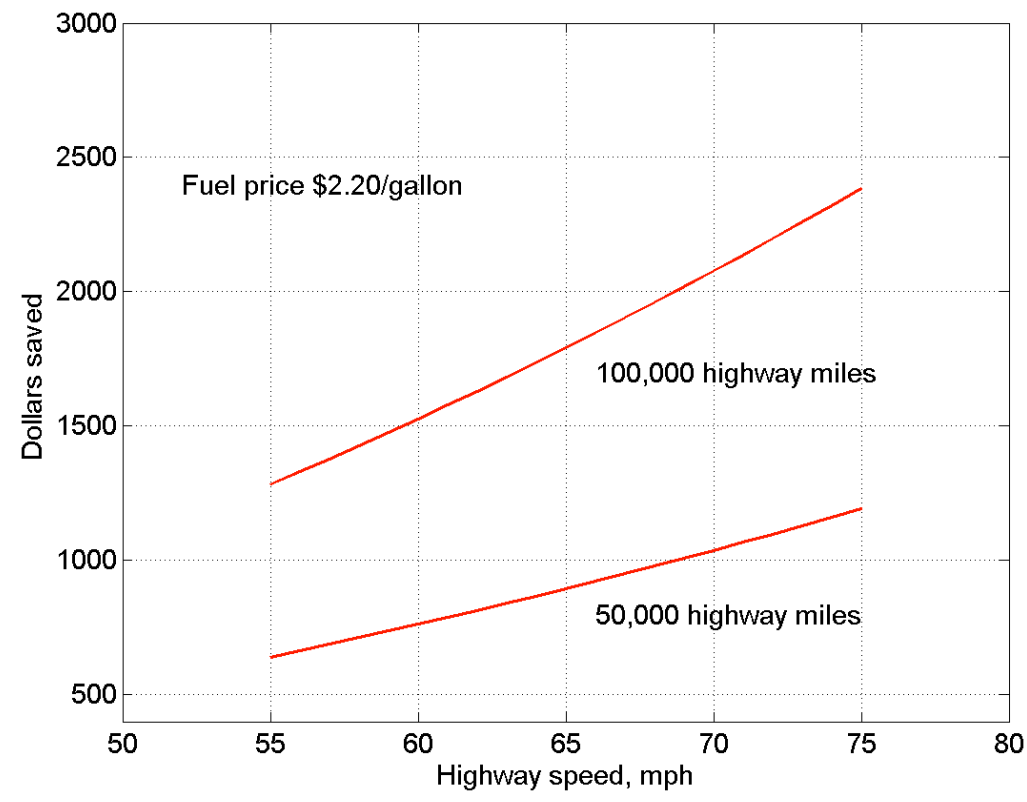

Figure 10. Potential dollar-savings from use of base flaps on a single trailer. 
These savings represent the increment associated only with the change in drag due to the presence or absence of flaps. The result will hold for any truck of similar size and shape and engine performance regardless of the loading of the truck or the rolling resistance.

The horizontal axis in Figure 10 is highway speed. Although the tests presented here are performed at $60 \mathrm{mph}$, the results are easily extrapolated to other speeds because the fuel needed to travel a given distance is quadratic in speed. Dollar savings from the use of flaps is greater at higher speeds, because aerodynamic drag is a larger fraction of the total resistance. However, total fuel consumption will increase with increasing speed. 


\title{
APPENDIX C
}

\section{Continued Development and Improvement of Pneumatic Heavy Vehicles}

\author{
Principal Investigator: Robert J. Englar \\ Georgia Tech Research Institute (GTRI) \\ Atlanta, GA 30332-0844 \\ (770) 528-3222; fax: (770) 528-7077; \\ e-mail: bob.englar@gtri.gatech.edu
}

Technology Development Manager: Sid Diamond

(202) 586-8032,sid.diamond@ee.doe.gov

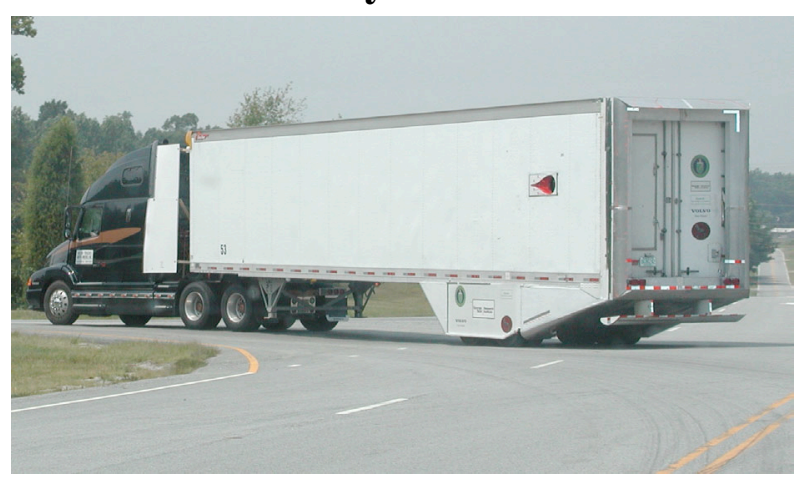

Technical Program Manager: Jules Routbort

(630) 252-5065, routbort@anl.gov

Contractor: Dept. of Energy - National Energy Technology Laboratory, Morgantown, WV

Contract No.: DE-AC26-02EE50691

\section{Objective}

- Previous smaller-scale model wind-tunnel evaluations at GTRI had demonstrated up to $15 \%$ reduction in aerodynamic drag coefficient due to blowing and $10-12 \%$ due to the device's corner rounding for a combined drag reduction of $25-27 \%$, not accounting for the fuel use by the blower. However, these tunnel results had translated into less-than-expected fuel economy increases during full-scale on-track testing. Reasons for this difference need to be determined, and improved aerodynamic characteristics identified.

- Continue this PHV technology development by employing new wind-tunnel results to modify the PHV test trailer. Conduct additional fuel economy evaluations of the blown test vehicle to confirm improved drag reduction for parasitic energy loss reduction, fuel economy improvement, reduced emissions, and increased safety of operations for Heavy Vehicles.

\section{Approach}

- Conduct additional experimental evaluations of modified wind-tunnel models to enhance the pneumatic aerodynamic capabilities of the PHV configurations.

- Use these results to modify the DOE full-scale pneumatic test vehicle.

- Conduct preliminary on-road testing followed by on-track SAE Type-II fuel economy tests of the PHV test vehicle to verify and improve the drag-reduction and fuel-economy-increasing properties.

- Identify pneumatic aerodynamic and geometry improvements to increase fuel economy by an additional factor of 2 to 3 over that exhibited during our earlier Phase I SAE Type-II fuel-economy tests.

\section{Accomplishments}

- Wind-tunnel tests of our modified smaller-scale PHV model have shown improved drag reductions for the more-realistic current configuration with real-world components such as axles, springs, under-ride bar, jack stands, etc. These tests confirmed that this new blown PHV model could reduce drag coefficient by $31 \%$ below that of the baseline HV configuration, with major geometry modification and not accounting for fuel use for blowing. New tests also demonstrated that active blowing control can reduce side-wind effects, and that the device has the potential to assist in braking and safety of operation for Pneumatic HVs. 
- Improvements thus needed for the Phase II full-scale PHV track test were identified; and the new test trailer design and modifications were completed. Primary here were improved fairings leading into the blown surfaces, improved blowing surfaces, and improved cab gap extenders.

- On-road and on-track SAE Type-II testing were completed. Results show increased improvement in fuel economy due to both blowing and the physical geometry of the new PHV truck. Fuel Economy Increase ( $\% \mathrm{FEI}$ ) of up to $4.5 \%$ at $65 \mathrm{mph}$ due to blowing only, not accounting for fuel used by the blower.

\section{Future Direction}

- Conduct additional development and demonstrations of pneumatic aerodynamic drag-reduction, braking, fueleconomy and safety of operation techniques to provide a confirming database allowing application of this technology to operational Pneumatic Heavy Vehicles. Interact with DOE and Truck Manufacturers Association's test and evaluation of these devices on their operational rigs.

\section{Introduction}

Since aerodynamic drag is the major component of Heavy Vehicle (HV) resistance at highway speed and thus strongly impacts related fuel economy, GTRI has been applying advanced aircraft aerodynamic technology using blowing to reduce that drag generated by these bluff-based high-drag vehicles. Using the pneumatic aerodynamic technology known as Circulation Control [Ref. 1] and certain geometry changes, we have been able to reduce drag coefficient $\left(\mathrm{C}_{\mathrm{D}}\right)$ on $\mathrm{HV}$ models by up to $15 \%$ reduction in aerodynamic drag coefficient due to blowing and $10-12 \%$ due to the device's corner rounding, for a total of $25-27 \%$, not accounting for fuel use by the blower (see Figure 1) during a 5-year tunnel test program for DOE [Refs. 2, 3, 4]. Of further advantage, we could also increase drag as needed for braking during downhill operation without any moving aerodynamic parts by blowing only select trailing-edge surfaces on the trailer. We could also potentially reduce the huge drag increase and loss of stability which occur when an HV experiences side winds or gusts. This multi-function potential of the blown configurations is seen in the wind tunnel data of Figure 1. Possible compressed air sources are an HV tractor's turbocharger or an auxiliary engine similar to a refrigeration unit.

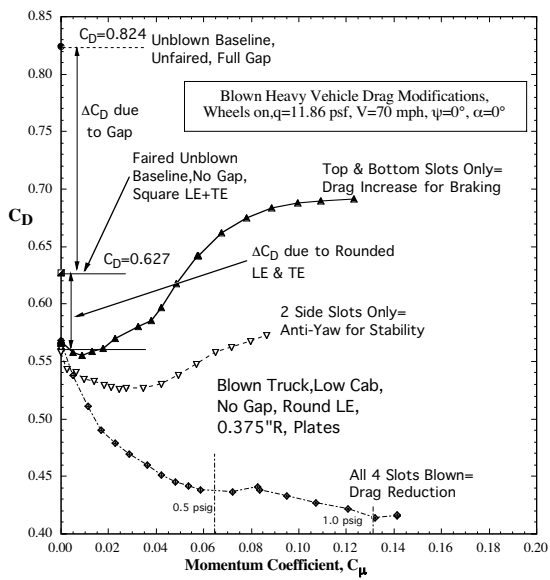

Fig. 1- Drag reduction or drag increase demonstrated by earlier GTRI GTS generic model PHV, depending on blowing slot activated

Full-scale fuel economy tests were conducted [3, 4] during our previous DOE program. These SAE Type-II official test-track results on a Pneumatic HV (PHV) configuration somewhat different from the tunnel models showed measured $\%$ FEI of only $4 \%$ $5 \%$, not accounting for energy use for blowing. Since that first SAE test, the current program has thus concentrated on: determining the difference between wind-tunnel results and the less-than-expected fullscale performance; correcting the blown configuration problem areas; and preparing for a second fueleconomy evaluation with the improved PHV vehicle. 


\section{Experimental Details and Results for Updated Wind-tunnel Model}

Experimental wind-tunnel developments of this technology conducted on a smaller-scale PHV model under previous DOE funding [Refs. 2, 3, 4, 5] had led to two full-scale on-road Tuning Tests plus an SAE Type-II Fuel Econ-omy Test conducted at the 7.5-mile test track at Transportation Research Center in Ohio, with the results reported above. However, the wind-tunnel model employed here (the very generic Ground Transportation System, GTS, config-uration modified with our blowing systems, Refs. 2 and 3) was geometrically quite different from the actual on-road and on-track test PHV configuration. This simple model had generated drag reductions up to $84 \%$ relative to a stock trailer configuration (Ref. 5). Since the fuel economy increases from these drag reductions were found to be less for the track-test PHV vehicle than the tunnel data predicted, we returned to the tunnel this past year to determine the reasons and possible corrections on a model modified to be very similar to the fullscale blown test truck. These results were reported in Refs. 6 and 7, and are summarized here to demonstrate the significance of certain aerodynamic components and features.

The new PHV model fabricated and tested is shown in Figure 2, where many of the new components are noted. At DOE request, we replaced the earlier generic GTS tractor with the more current Generic Conventional Model.

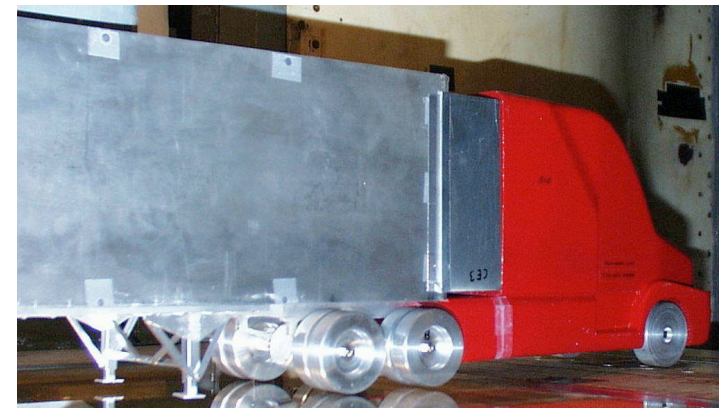

Fig. 2- New GCM tractor model with full cab extender (CE3"), 4 stock wheels per axle, jack stands, and differentials

(GCM) tractor shown in Figure 2. Note that while this tractor model is representative of current onroad vehicles, it is not specific to any one brand. The new trailer has blowing surface components similar to before but covering less vertical length (the trailer floor is raised to the conventional level, not "low-boy" height). The model has many new components typical of the real test vehicle:

Trailer suspension, springs, brakes, axles, support feet (jack stand), I-beam floor rails

Tractor differentials and suspension

Mirrors

Cab gap extenders (full or $60 \%$ coverage)

Trailer rear under-ride bar and mud flaps

Stock wheels spaced 4 per axle, plus other wheel options

Details of some 325 new wind-tunnel runs conducted over ranges of tunnel speed, blowing rate, yaw (side wind) angle and model configuration variations are presented in References 6 and 7, and the most significant findings are presented below.

The importance of cab extenders in counteracting the adverse effects of asymmetric vortex shedding in the gap between tractor and trailer is shown in Figure 3 for the unblown tractor/trailer configuration. Clearly, a gap fairing is needed here (see "Full Open Gap" curves), but since 100\% fullcoverage gap ex-tenders (CE3") are not practical during real vehicle turning, we decided on a $60 \%$ gap closure (CE1.5") which produces nearly the same aerodynamic drag results as the $100 \%$ full closure but is functionally feasible (it leaves a 16 " gap on the real vehicle).

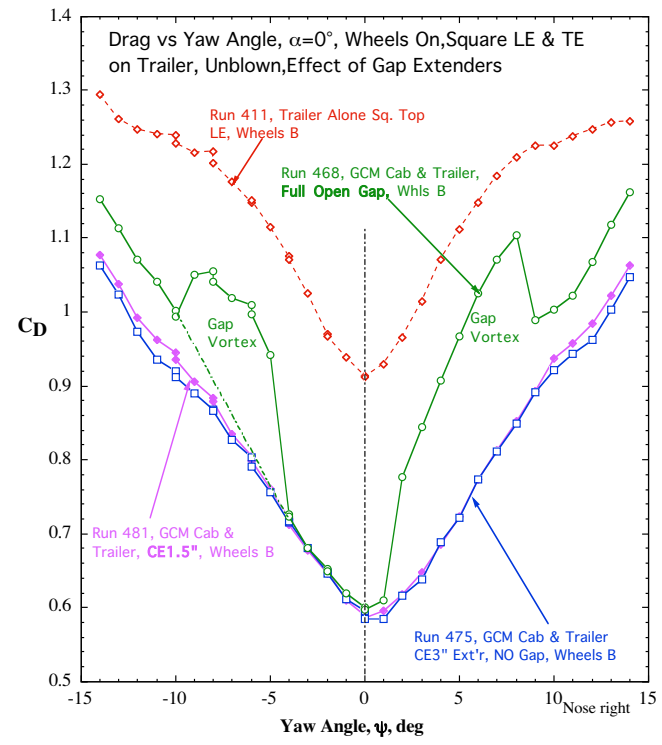

Fig. 3- Measured drag of the new trailer and the tractor/trailer combo at yaw angle for various gaps 
Figure 4 shows the results of blowing and various components on drag reduction as compared to the conventional GCM model with square leading and trailing edges on the trailer and a full open gap. Run 171 is the best blown configuration of the GTS "unrealistic" generic model from the previous tests.

The top curve (Run 585) of Fig. 4 represents the corresponding blown trailing edge geometry with the representative 4-per-axle wheels and suspension installed on the GCM PHV model. Initial drag reduction due to $\mathrm{Cm}$ flattens out and then rises slightly as the wake from the wheels interferes with the jet turning, much as it did on the full-scale PHV of Ref. 4. As we faired the wheels, (Run 601), the conditions improved until the new blown truck was very close to the dashed target curve from the previous generic tests, Run 171. This new configuration without the underfloor disturbance yields a drag reduction of $24 \%$ below the unblown baseline (Run 467) at the expected full-scale blowing coefficient of $\mathrm{Cm}=0.065$. Note however, that if the trailer-wheel wake effects were eliminated entirely (Run 584), and if the floor I-beams were faired over (Run 604), considerably better $C_{D}$ reduction will occur. In the extreme, if the aft tractor wheel effects were eliminated as well (Run 605), a drag coefficient of $\mathrm{C}_{\mathrm{D}}=0.33$ is possible for this PHV configuration-this is on the order of current sports car coupes. This further emphasizes the strong influence of the vehicle undercarriage. These results need be considered by other researchers conducting drag reduction efforts on current HVs: it is very important to account for the underbody and wheels of the entire vehicle!

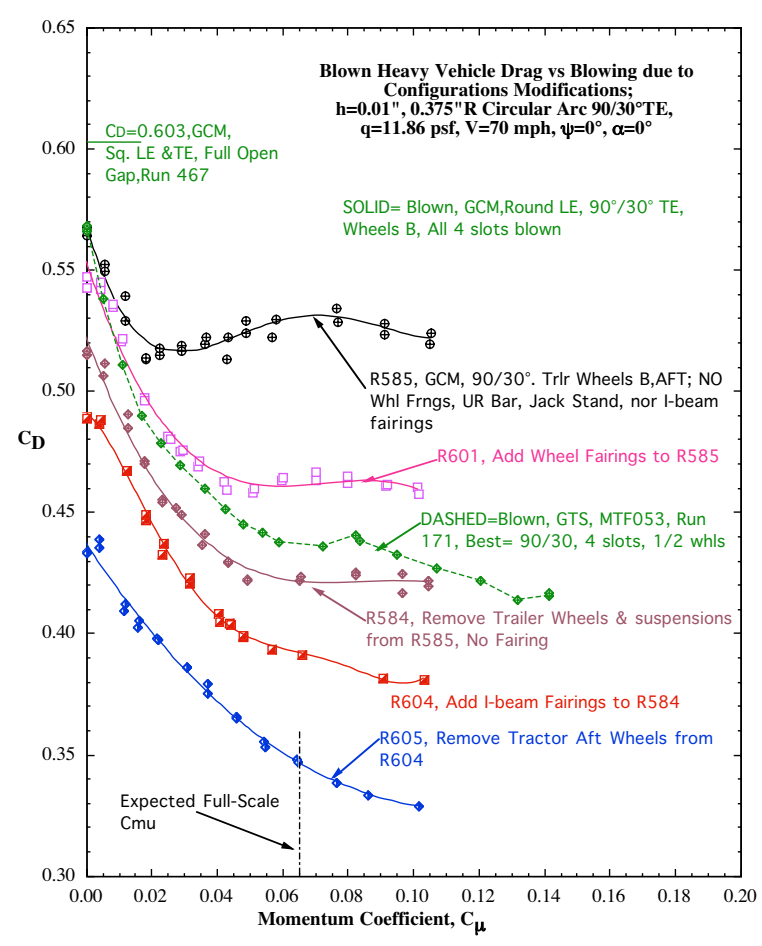

Figure 4- Measured drag reduction due to blowing all 4 slots of various configurations

Fairings covering the trailer suspension, axles and wheels were tested, thus eliminating many of the undercarriage problems from Fig. 4. Figure 5 shows the drag reductions of the faired configuration compared to the "baseline" $\mathrm{HV}$ which has a drag coefficient $C_{D}=0.702$. The blown results, which are due to several variations in slot height, are seen here. While all configurations have the same slot height on the top and sides, the bottom surface slot height is varied here to determine any gains from improving the disturbed lower surface flow by adding more mass flow there. Indeed, it is seen that increasing the lower slot height does reduce $C_{D}$ at the same $C \mu$. In the extreme, too large a bottom slot can reverse this trend. For the best arrangement, $\mathrm{C}_{\mathrm{D}}$ is reduced $31 \%$ at $\mathrm{C} \mu=0.04-0.05$ relative to the stock baseline configuration $\left(C_{D}=0.702\right)$, with major geometry modification and not accounting for fuel use for blowing. Thus this latest wind-tunnel evaluation has provided a "real-world" configuration that should be capable of about 15-16\% fuel economy increase at highway speeds. These tests also confirmed the ability of blowing to provide yawing moment to counteract side winds and thus provide directional stability to these large-sided vehicles (see Ref. 7). A valuable lesson learned from these tests was the considerable interference (separated and reversed flow) 
effects produced by all the mechanical components and the wheels on the trailer underside.

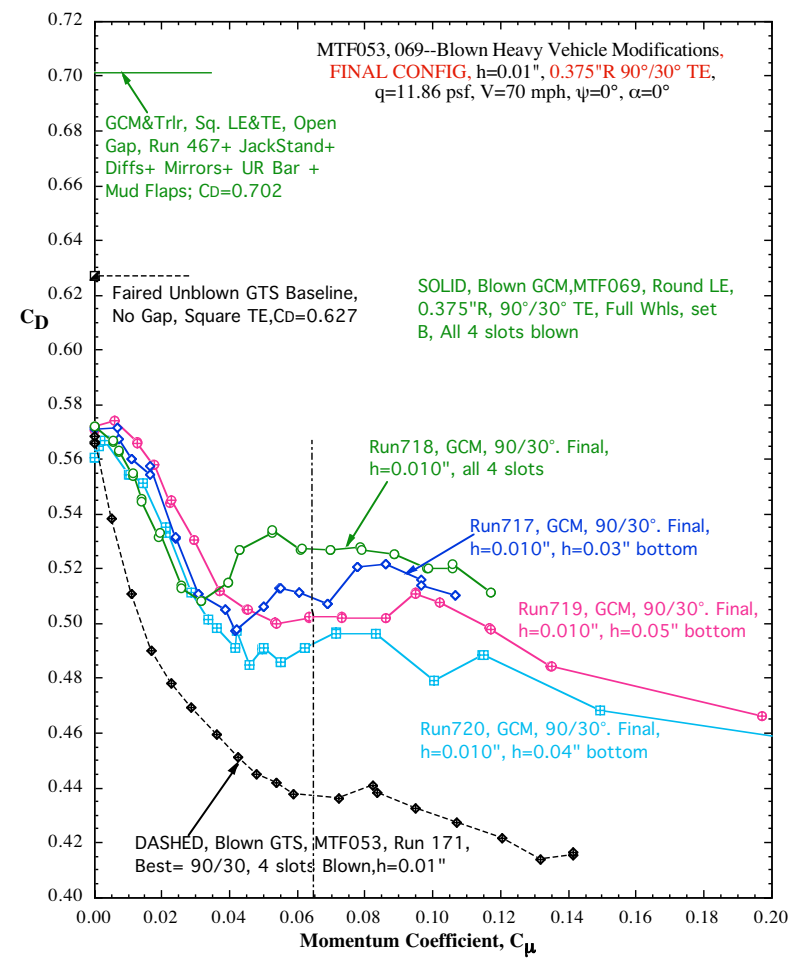

Fig. 5- Drag reduction due to blowing and geometry on the final PHV configuration

One last item of interest from these tests is the additional drag and incremental horsepower required to overcome the protrusions into the flow of the components shown in Table I.

\begin{tabular}{|l|c|c|}
\hline \multicolumn{1}{|c|}{ Component } & $\Delta \mathrm{C}_{\mathrm{D}}$ & $\Delta \mathrm{HP}, 70 \mathrm{mph}$ \\
\hline \hline Rear View Mirrors & +0.043 & +10.51 \\
\hline Under-ride bar & +0.049 & +11.97 \\
\hline Mud flaps & +0.005 & +1.22 \\
\hline Jack stands (feet) & +0.002 & +0.49 \\
\hline Tractor Different'l & +0.001 & +0.24 \\
\hline
\end{tabular}

Table I- Drag increments and corresponding required HP due to external components

Figure 5 thus represents the drag of the fullscale PHV test vehicle configuration, which eliminates the major Table I component items (under-ride bar and flaps are now enclosed) but still is hampered by the presence of the required rear-view mirrors.

\section{Full-Scale Trailer Modifications}

As a result of the above series of tunnel evaluations and developments, a final blown Pneumatic Heavy Vehicle configuration to undergo fuel economy testing was determined, and includes the following:

- Ninety-degree (vertical side corners) and 30-degree (top \& bottom) blowing surfaces with variable slot heights

- 60\% Cab Extender, 16" gap exposed

- Wheel and axle fairings on trailer, and no exposed trailer mud flaps

- Forward trailer wheel location

- Aerodynamic under-ride bar (airfoil fairing)

- Stock wheels, four per axle on trailer

- Stock differentials, axles, and springs on tractor

- Side mirrors on tractor, as required

The trailer modification was completed by GTRI and our teammate prototype shop Novatek, Inc. in early Summer 2004. It is shown at the test track in Figure 6. Not shown are the internal blowers connected by ducting to the trailing edge blowing surfaces, nor the internal diesel drive motor powering these blowers. Air was entrained into these blowers through the NACA inlets on the trailer sidewalls shown in Figure 6. Preliminary checkout testing was conducted at GTRI to measure internal and jet pressures, temperatures, and flow rates, degree of trailing edge jet turning, and data systems operation. When all systems where confirmed, the PHV trailer was picked up by teammate Volvo Technology of America (VTA) and transported to Volvo's facility in North Carolina.

\section{Preliminary Tuning Test 3 (TT3)}

After arrival at the Volvo facility, the test truck was again evaluated to assure blowing jet turning, which proved quite satisfactory, especially on the 90 degree vertical surfaces. Tuning Test 3 (TT3) was then conducted on a four-lane highway to confirm that all blowing and data systems were operating successfully on-road, and to generate preliminary fuel consumption data prior to the upcoming SAE Type-II test. On-road flow field attachment due to jet turning was very similar to that shown in Figure 6 with blowing ON. With blowing OFF, the tufts pointed aft and fluttered. This photo gives a graphic demonstration of the blowing effectiveness in 
preventing aft-surface flow separation on the trailer aft corners.

Although not considered as truly indicative of fuel economy determination, these on-road tuning tests we conducted yielded significant and informative trends. To eliminate any side wind or elevation effects, they were run in both North/South directions on a 2.9-mile length of four-lane highway using an on-board digital fuel readout based on recorded pulses of the Volvo diesel engine's fuel-injection system. Speed was set and maintained by the Volvo cruise control at $65 \mathrm{mph}$ between preset road signposts once the vehicle had achieved test speed, so no accelerations/ decelerations were included. Onboard laptop computers recorded truck engine parameters and fuel consumption plus blowing parameters. The data was averaged over the N/S runs to yield each test point and then each test condition was repeated at least once for consistency (a total of 29 runs were conducted in three days in August 2004).

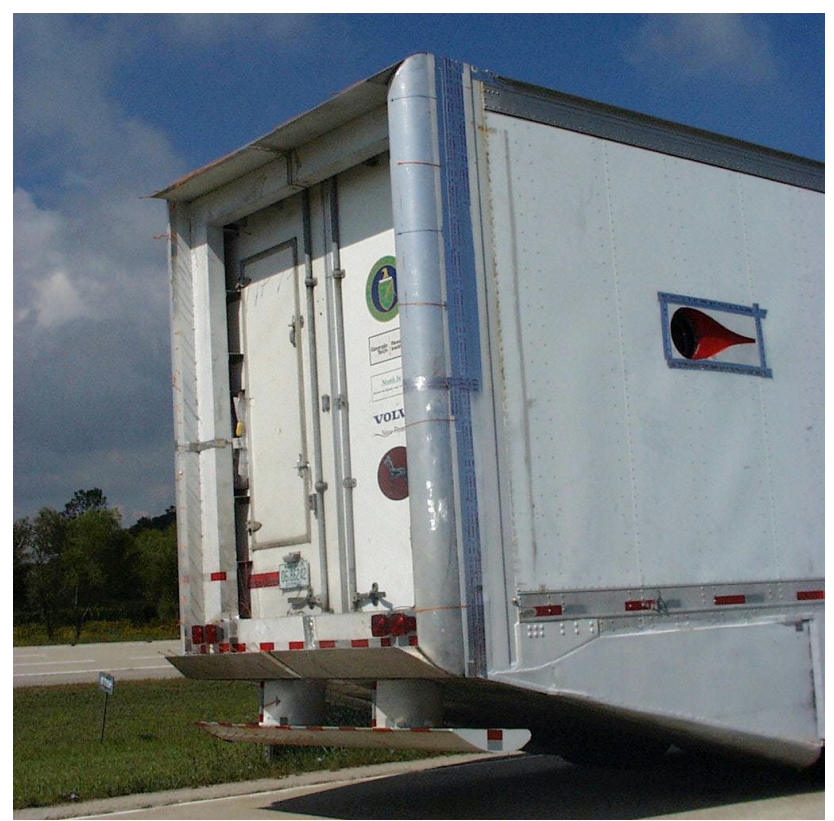

Fig. 6- Tuft flow visualization showing flow turning with blowing ON

Fuel economy was determined using several methods, with results shown in Figure 7 as functions of blowing momentum coefficient, $\mathrm{C} \mu$. (Data are plotted as percent miles/gallon change from the mpg of the baseline stock trailer, which was also tested with the same tractor.) The engine parameters were recorded digitally and integrated to give time-averaged mpg. The data shown in Fig 7 , labeled "full distance", was integrated over the entire 2.9 mile run, averaged in each direction. This timeaveraged data (striped bars) is compared here with the trends of the wind-tunnel data "MTF069" (this tunnel data has been converted to \%mpg increase by assuming that $\% \mathrm{C}_{\mathrm{D}}$ reduction is roughly twice the \%fuel economy increase; see Refs. 2, 3, 4). This "onroad" integrated data thus shows the \%Fuel Economy Increase to range from $4-5 \% \pm 1 \%$.

This preliminary Tuning Test 3 was thus completed, and though not considered an official fuel economy test, it confirmed that the PHV blown test rig was yielding appropriate drag reduction characteristics due to blowing and was thus ready for SAE Type-II fuel economy testing.

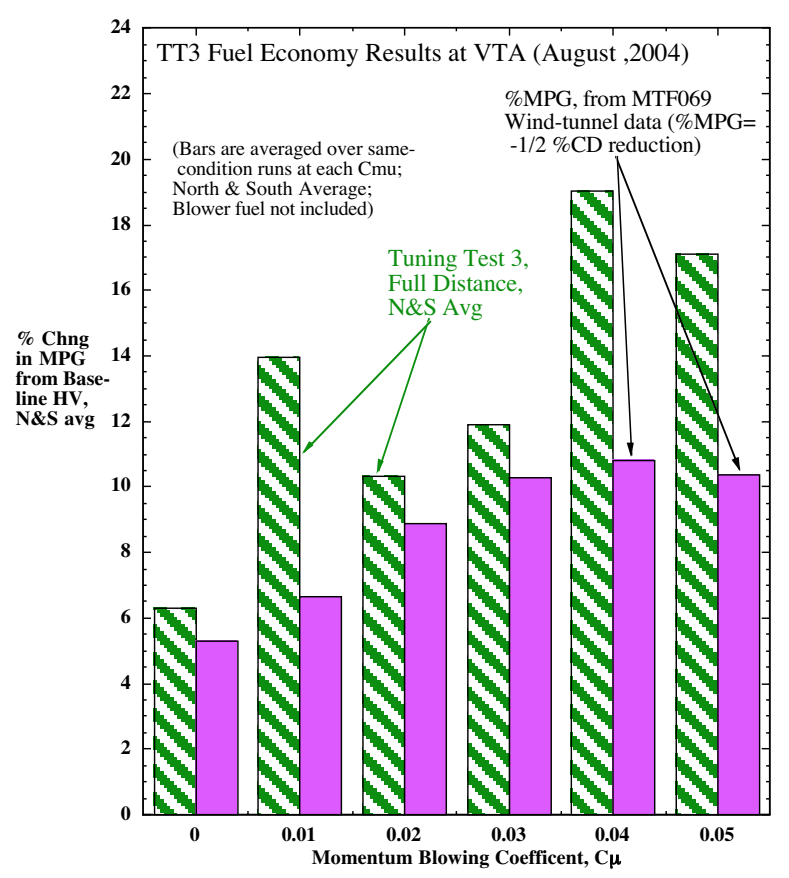

Fig. 7- Tuning Test 3 Fuel economy results and windtunnel comparisons 


\section{Phase II SAE Type-II tests}

The pneumatic test truck and a stock reference (control) tractor/trailer were trans-ported by Volvo to the Transportation Research Center (TRC) 7.5mile test track in East Liberty, $\mathrm{OH}$ for our Phase II SAE Type-II fuel economy evaluations. These tests were conducted by TRC drivers and personnel in strict accordance with SAE J1321 procedures, Ref. 8. For each valid test point of fuel consumed, these require that 3 successive runs of 6 laps each (45 miles around the TRC test oval), at a constant speed and constant blowing parameters or test configuration, be made by the test (T) truck and by the control (C) truck at the same time within certain allowable departure times and displacement distances. Fuel economy is measured by weighing removable fuel tanks and then comparing the Test truck's fuel burned to the Control truck's. This eliminates variations in temperature, side winds, etc. When the Test/ Control fuel-burned ratio is within a required consistency of each other for 3 measured runs, that data point is considered valid. A view of the entire PHV test vehicle, including the added $60 \%$ cab extenders and wheel fairings, is seen in Figure 8. The Control tractor-trailer was a second Volvo/Great Dane combo with stock geometry, also shown in Fig. 8.

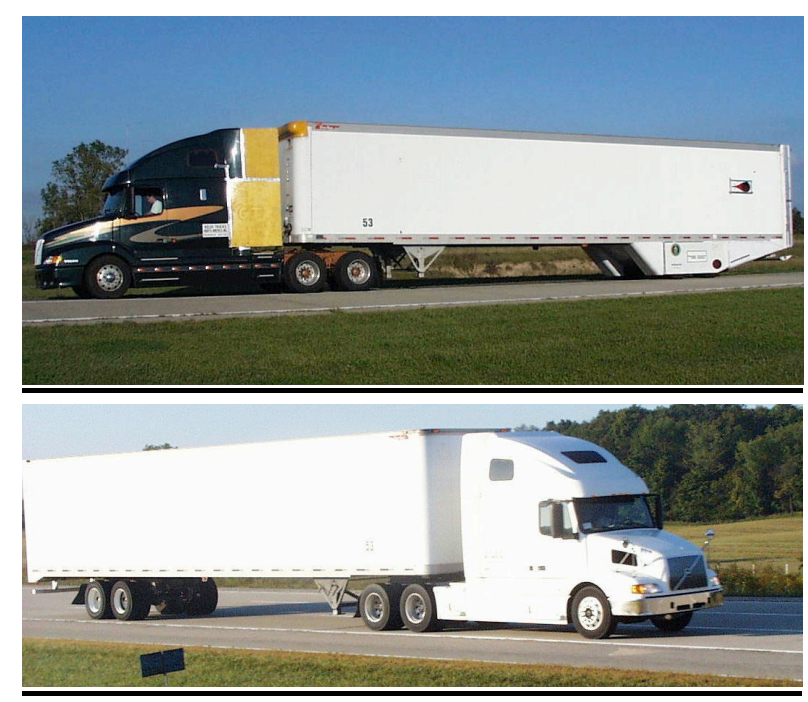

Fig 8- PHV test trailer (top) at TRC with rebuilt cab extenders and Control trailer (bottom)

For these test runs, our target data points based on TT3 and Figure 7 were $\mathrm{C} \mu=0.0 .0 .02$ and
0.04 at $65 \mathrm{mph}$ speed and $\mathrm{C} \mu=0.0,0.02$, and 0.035 at $75 \mathrm{mph}$. Plus, for comparison as a baseline vehicle, the PHV test truck had to be disassembled on-site and returned to the baseline (stock) configuration, and then run at 65 and $75 \mathrm{mph}$ also. This test program was conducted at TRC in September 2004.

The \%Fuel Economy Increase ratios (\%FEI, same as $\%$ MPG increase) come from comparing the $\mathrm{T} / \mathrm{C}$ fuel-burned ratios for each test condition to the $\mathrm{T} / \mathrm{C}$ of the stock baseline truck at the same speed (Ref. 8). These TRC fuel economy increases are seen for the two test vehicle speeds in Fig. 9 ("PHV Total", top solid curves), also compared to the GTRI wind-tunnelbased data from Fig 7. The TRC data have a very similar trend to the tunnel data concerning increased $\%$ FEI with blowing $\mathrm{C} \mu$. The lower solid curves show $\% \mathrm{FEI}$ due to blowing only, where up to $4.5 \% \mathrm{FEI}$ is seen, not accounting for fuel use by compression. Higher blowing than 0.03 seems to cause a slight drop in fuel economy, just as it did in the tunnel data at higher $\mathrm{C} \mu$, probably due to lack of flow disturbance underneath the trailer and degradation of the effects of higher blowing (see Fig. 5 as well). However, relative to our previous SAE test on the first generation of this PHV test truck (Refs. 3, 4, 5), these results are more than 2.2 times those earlier \%FEI results. To put things in perspective, $1 \%$ increase in FEI represents approximately 220-240 million gallons of diesel fuel saved by the US heavy vehicle fleet each year.

In neither sets of the above data is the fuel used to power the blower engine yet included. In the Fig. 9 middle (dashed) curves we have included blower fuel burned, where we have also added the use of pulsed (cyclic) blowing to reduce the blowing mass flow required to achieve these drag reductions (see Ref. 9 for details of this technology which GTRI developed with NASA). Results including this not-yet-optimized system still show approximately $8-9 \% \mathrm{FEI}$ for these blown configurations including blower fuel. Note also in Fig. 9 that the data at the higher speed $(75 \mathrm{mph})$ show greater improvement from blowing than $65 \mathrm{mph}$ since drag there is the more dominant term over rolling resistance. As noted, the raw TRC data have been equalized to assure that the $\mathrm{T} / \mathrm{C}$ ratios for the baseline reference configurations (from which the test vehicle fuel economy increasaes were derived) were the same (1.0) at both speeds. 


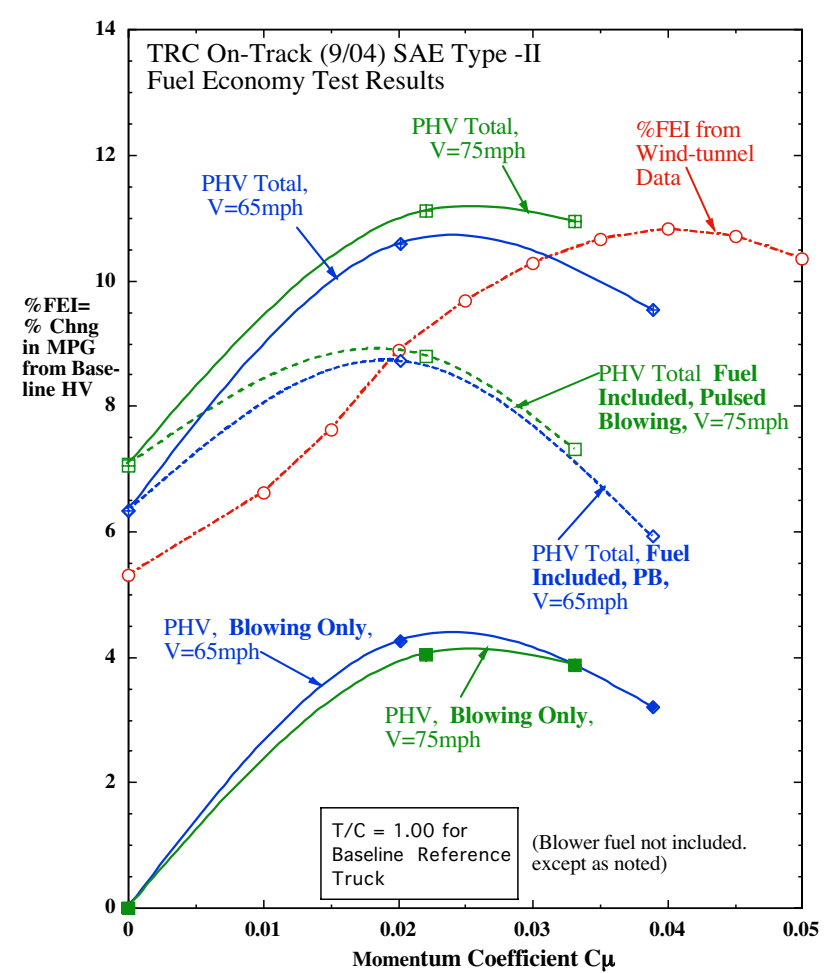

Fig 9 - TRC SAE Type-II fuel economy results for the pneumatic system components

\section{Conclusions}

To advance the state of development of pneumatic aerodynamics for Heavy Vehicle drag reduction, fuel economy, braking, stability and safety of operation, GTRI and its team members have continued in 2004 our previous program for DOE EERE. We have conducted new modelscale wind-tunnel investigations to identify and correct aerodynamic problem areas from our first fuel-economy test. We have also completed new full-scale on-road and test-track fuel economy validations of these advanced capabilities on a full-scale Pneumatic Heavy Vehicle. Results of this recent effort include:

- We have identified aero problem areas existing after our first PHV road test and how to correct these; current wind tunnel data indicate that drag reductions of up to $31 \%$ result from the new "realworld" PHV configuration, from which fuel economy increases of about $15-16 \%$ due to blowing and associated geometry improvements should result at highway speeds. Based on this wind-tunnel data, a new blown test vehicle modification was fabricated and assembled.

- SAE Type-II fuel economy runs of this new PHV vehicle on the TRC test track showed up to $4.5 \%$ at 65 mph due to blowing only, not accounting for fuel used by the blower.

- The Pneumatic Heavy Vehicle concept has now been verified both by smaller-scale wind tunnel evaluations and by full-scale on-road and on-track SAE testing to be a promising means to reduce drag and increase fuel economy of HVs. We must still address and resolve the problems caused by undercarriage and wheel component disturbances that seem to cause lesser blowing effectiveness at higher blowing.

\section{$\underline{\text { References }}$}

1. Englar, Robert J., "Circulation Control Pneumatic Aerodynamics: Blown Force and Moment Augmentation and Modification; Past, Present and Future," AIAA Paper 2000-2541, June, 2000.

2. Englar, R. J., "Advanced Aerodynamic Devices to Improve the Performance, Economics, Handling and Safety of Heavy Vehicles," SAE Paper 2001-01-2072, May 14-16, 2001.

3. Englar, R. J. "Pneumatic Heavy Vehicle Aerodynamic Drag Reduction, Safety Enhancement, and Performance Improvement," Proceedings of the UEF Conference "The Aerodynamics of Heavy Vehicles: Trucks, Buses, and Trains”, Dec. 2002.

4. Englar, R. J. "Drag Reduction, Safety Enhancement and Performance Improvement for Heavy Vehicles and SUVs Using Advanced Pneumatic Aerodynamic Technology", SAE Paper 2003-01-3378, Nov. 2003.

5. Diamond, Sidney, "FY2003 Annual Progress Report for Heavy Vehicle Systems Optimization, FreedomCAR and Vehicle Technologies Program", February, 2004.

6. Englar, R. J., "Continued Development \& Improvement of Pneumatic Heavy Vehicles, Phase VI, DOE Quarterly Report 7," April 15, 2004.

7. Englar, R. J., "Continued Development \& Improvement of Pneumatic Heavy Vehicles, Phase VI, DOE Quarterly Report. 8,” July 15, 2004. 
8. "Joint TMC/SAE Fuel Consumption Test Procedure-Type-II," SAE J1321, Oct 86.

9. Jones G.S. and R.J. Englar, Advances in Pneumatic High-Lift Systems through Pulsed Blowing," AIAA Paper 2003-3411, June, 2003. 


\title{
APPENDIX D
}

\section{Heavy Vehicle Aerodynamic Drag: Experiments, Computations, and Design}

\author{
Kambiz Salari, Jason Ortega, Paul Castellucci, Craig Eastwood, Rose McCallen, Kipp Whittaker \\ Lawrence Livermore National Laboratory \\ 7000 East Ave, L-098, Livermore, CA 94551 \\ 925-423-0958, Fax 925-422-3389, mccallen1@llnl.gov
}

Technology Development Area Specialist: Sidney Diamond

(202) 586-8032; fax: (202) 586-1600; e-mail: sid.diamond@ee.doe.gov

Technical Program Manager: Jules Routbort

(630)252-5065; fax: (630)252-4289; email: routbort@anl.gov

Contractor: $\quad$ Lawrence Livermore National Laboratory

Contract No.: EEW0046

LLNL's effort consists of four experimental, computational, and design focus areas:

- An Experimental Study of Drag Reduction Devices for a trailer Underbody and Base

- Investigation of Predictive Capability of RANS to Model Bluff Body Aerodynamics

- Splash and Spray Suppression

- Computational Investigation of Aerodynamics of Rail Coal Cars and Drag Reducing Add-On Devices

The following describes the objective, approach, accomplishments, and future directions for each of these focus areas.

\section{A. An Experimental Study of Drag Reduction Devices for a Trailer Underbody and Base}

\section{Objective}

- This wind tunnel study investigates optimization of trailer base flaps and alternate forms of trailer skirts in an effort to reduce the aerodynamic drag of heavy vehicles, thereby improving their fuel efficiency.

\section{Approach}

- Low speed wind tunnel measurements are made on a $1 / 16^{\text {th }}$ scale generic tractor-trailer model at a widthbased Reynolds number of 325,000.

- The model is fixed to a turntable, allowing the yaw angle to be varied between $\pm 14^{\circ}$ in $2^{\circ}$ increments.

- Various add-on drag reduction devices are mounted to the model underbody and base.

- The wind-averaged drag coefficient at $65 \mathrm{mph}$ is computed for each configuration, allowing the effectiveness of the add-on devices to be assessed.

\section{Accomplishments}

- The most effective add-on drag reduction device for the trailer underbody is a wedge-shaped skirt, which reduces the wind-averaged drag coefficient by $2.0 \%$.

- For the trailer base, the most effective add-on drag reduction device is a set of curved base flaps having a radius of curvature of 0.91 times the trailer width. These curved base flaps reduce the wind-averaged drag coefficient by $18.8 \%$, providing the greatest drag reduction of any of the devices tested. 
- Maximum drag reduction for the angled base flaps occurs when the top and side flaps have deflection angles of $11.1^{\circ}$ and $10.1^{\circ}$, respectively.

- When the wedge-shaped skirt and curved base flaps are used in conjunction with one another, the windaveraged drag coefficient is reduced by $20 \%$.

\section{Future Direction}

- CFD simulations will be performed on a heavy vehicle with a wedge-shaped skirt to gain additional insight into the performance characteristics of the wedge-shaped skirts.

- When future track tests are performed using the angled base flaps, a recommendation will be made to test unequal deflection angles for the side and top base flaps.

\section{Introduction}

In an effort to improve the fuel efficiency of heavy vehicles, this wind tunnel study investigates the optimization of trailer base flaps and alternate forms of trailer underbody skirt designs. Previous research $^{1,2}$ on angled base flaps has demonstrated that this concept is capable of reducing the drag by as much as $10 \%$ on a heavy vehicle when the flaps are deployed at equal angular deflections. However, it is quite possible that an optimum configuration may be one in which the top and side base flaps have slightly different angular deflections. In the subsequent sections, we explore this possibility. Additionally, we investigate the drag reducing capability of curved base flaps, which have previously shown to perform about as well as straight base flaps. ${ }^{2}$ To circumvent the shortcomings of straight side skirts, which limit access to the trailer underside, we investigate three variations of a wedge trailer skirt concept that may provide the drag reduction benefits of straight side skirts.

\section{Experimental Setup}

The effectiveness of trailer underbody and base drag reduction devices is assessed by making axial force measurements in a wind tunnel on a $1 / 16^{\text {th }}$ scale generic tractor-trailer model (Fig. 1), which is a simple representation of a near-future tractor/trailer. The wind tunnel measurements are made in the NASA Ames Fluid Mechanics Laboratory open-circuit wind tunnel, which has a contraction ratio of $9: 1$, a test section size of 813 $\mathrm{mm} \times 1219 \mathrm{~mm}$, and freestream turbulence level of $0.15 \%$. The model measures $162 \mathrm{~mm} \times 225$ $\mathrm{mm} \times 1238 \mathrm{~m}$, giving a nominal width-based
Reynolds number of $R e_{w}=V w / v=325,000$, where $w$ is the model width $=162 \mathrm{~mm}, v$ is the kinematic viscosity of air, and $V$ is the freestream velocity. For each model configuration, the axial force measurements are made at yaw angles, $\psi$, ranging from $\pm 14^{\circ}$ in $2^{\circ}$ increments.

Four skirt designs (Fig. 2) are tested on the trailer underside: a long wedge skirt (Fig. 2a) which has an apex angle of $10^{\circ}$; a short wedge skirt (Fig. 2b) which has an apex angle of $22^{\circ}$; a short wedge skirt with an upstream center skirt (Fig. 2c); and two conventional straight side skirts (Fig. 2d), which are used as a reference for making performance comparisons with the other three skirt designs. A set of angled flaps is tested as a means of reducing the base drag of the model (Fig. 3a). The four curved base flap devices are constructed using a rapid prototyping technique (selective laser sintering), which forms a singlepieced design. The curved base flaps extend a distance of $48 \mathrm{~mm}$ from the trailer base and have radii of curvature, $R$, of $52,79,148$, and $288 \mathrm{~mm}$. In units of the trailer width, the radii of curvature are $0.32 w, 0.49 w, 0.91 w$, and $1.78 w$.

\section{$\underline{\text { Results }}$}

The wind-averaged drag coefficients, ${ }^{3} C_{d w a}$, for the four trailer skirts are listed in Table 1. It should be noted that the trailer skirts Cooper ${ }^{1}$ tested yielded reductions in the wind-averaged drag coefficient that are much larger than those shown presently. The reason for this difference may be due to the fact that the models Cooper used had complete axles and wheels on the trailer, which would likely contribute a much greater portion to the overall vehicle drag than simple 
half-cylinder wheels. Hence, the installation of the skirts on the more realistic models could result in a greater drag reduction. Both short wedge skirts provide negligible reduction of the windaveraged drag coefficient. On the other hand, it can be seen that the long wedge skirt provides the greatest drag reduction of the four trailer skirt designs. This suggests that the long wedge skirt is a design that can improve upon that of the traditional straight side skirts by not only yielding a greater reduction in the wind-averaged drag coefficient, but also allowing easier access to the trailer underside. However, additional testing of the long wedge skirt with a more realistic trailer underbody and a moving ground plane is needed before a definite conclusion can be drawn.

The angled base flaps on the sides and top of the trailer are tested at independent angular deflections of $5^{\circ}, 10^{\circ}, 15^{\circ}$, and $20^{\circ}$, while the bottom flap is maintained at a deflection angle of $0^{\circ}$. It is seen in the data that there is a combination of $a_{\text {side }}$ and $a_{\text {top }}$ that yields a minimum in the windaveraged drag coefficient in the vicinity of $a_{\text {side }} \approx$ $10^{\circ}$ and $a_{\text {top }} \approx 10^{\circ}$. The wind-averaged drag coefficients in the range of $5^{\circ} \leq a_{\text {side }} \leq 15^{\circ}, 5^{\circ} \leq a_{\text {top }}$ $\leq 15^{\circ}$ are fit with a second-order polynomial surface to estimate the angular deflections that yield a minimum value of the wind-averaged drag coefficient. Doing so gives a minimum in the polynomial surface at $a_{\text {side }}=10.1^{\circ}$ and $a_{\text {top }}=11.1^{\circ}$, at which location the wind-averaged drag coefficient is $0.493 \pm 0.004$. Table 1 shows that this value is $16.4 \pm 0.8 \%$ less than that of the baseline configuration.

As a comparison to the base flaps made of straight plates, four curved base flap configurations are tested. The minimum measured wind-averaged drag coefficient occurs for the curved base flaps that have a dimensionless radius of curvature of $R / w=0.91$. As shown in Table 1, the wind-averaged drag coefficient for this configuration is $18.8 \pm 0.8 \%$ less than that of the baseline case. This reduction in the drag coefficient is the largest for any single add-on device tested in this study.

Having analyzed the performance of the trailer skirts and base flaps on an individual basis, we now assess the effectiveness of combinations of these devices and determine the configuration that results in the greatest drag reduction. Of all the devices tested, the combination of the curved

\begin{tabular}{|l|c|c|}
\hline \multicolumn{1}{|c|}{ Configuration } & $\begin{array}{c}C_{d w a} \\
( \pm 0.004)\end{array}$ & $\begin{array}{c}\% \text { Reduction in } \\
C_{d w a}( \pm 0.8 \%)\end{array}$ \\
\hline Baseline & 0.590 & - \\
\hline $\begin{array}{l}\text { Baseline w/ long } \\
\text { wedge skirt }\end{array}$ & 0.578 & 2.0 \\
\hline $\begin{array}{l}\text { Baseline w/ short } \\
\text { wedge skirt }\end{array}$ & 0.587 & 0.1 \\
\hline $\begin{array}{l}\text { Baseline w/ short } \\
\text { wedge skirt and } \\
\text { center skirt }\end{array}$ & 0.587 & 0.1 \\
\hline $\begin{array}{l}\text { Baseline w/ straight } \\
\text { side skirts }\end{array}$ & 0.582 & 1.4 \\
\hline $\begin{array}{l}\text { Baseline w/ angled } \\
\text { base flaps }\left(\alpha_{\text {top }}=\right. \\
\left.11.1^{\circ}, \alpha_{\text {side }}=10.1^{\circ}\right)\end{array}$ & 0.493 & 16.4 \\
\hline $\begin{array}{l}\text { Baseline w/ curved } \\
\text { base flaps }(R / w= \\
0.91)\end{array}$ & 0.479 & 18.8 \\
\hline $\begin{array}{l}\text { Baseline w/ angled } \\
\text { base flaps }\left(\alpha_{\text {top }}=\right. \\
\left.10^{\circ}, \alpha_{\text {side }}=10^{\circ}\right) \text { and } \\
\text { long wedge skirt }\end{array}$ & 0.484 & \\
\hline $\begin{array}{l}\text { Baseline w/ curved } \\
\text { base flaps }(R / w= \\
0.91) \text { and long } \\
\text { wedge skirt }\end{array}$ & 0.472 & \\
\hline
\end{tabular}

Table 1. Wind-averaged drag coefficient, $C_{d w a}$, and the percent reduction in the wind-averaged drag coefficient relative to the baseline case for various model configurations.

base flaps $(R / w=0.91)$ with the long wedge skirt gives the greatest reduction in the wind-averaged drag coefficient. This combination results in a $20.0 \pm 0.8 \%$ reduction in the wind-averaged drag coefficient, the majority of which is due to the contribution of the curved base flaps. The windaveraged drag coefficients for this configuration and that of the combination of the long wedge skirt and the straight base flaps at $a_{\text {side }}=a_{\text {top }}=10^{\circ}$ are shown in Table 1.

A comparison of the wind-averaged drag coefficients of the model with trailer skirts and the model with base flaps indicates that the base flaps provide a substantially larger drag reduction than that of the skirts. Assuming that the trends in the wind-averaged drag coefficients are applicable to full-sale heavy vehicles, these results could have important implications for the current day trucking industry. Clearly, if a trucking fleet decided to purchase a single add-on drag device to obtain the greatest drag reduction, the choice of curved base 
flaps would be the best alternative. However, the angled base flaps may be more attractive from an investment point of view since their rather simple design would be less to manufacture and, thereby, require a smaller initial investment.

\section{Conclusions}

Through this study, we have investigated several add-on drag reduction devices. The windaveraged drag coefficient is used as a means of comparing the performance of trailer skirts and base flaps. Of the trailer skirts that are tested, the long wedge skirt provides the greatest drag reduction. The angled and curved base flap devices yield reductions in the wind-averaged drag coefficient approximately eight to nine times greater than that of the long wedge skirt. It should be noted that the results of this wind tunnel study are valid for the low Reynolds number regime that was tested. Future tests will be conducted on actual heavy vehicles to determine the influence of Reynolds number and the moving ground plane beneath the vehicle.

What is needed to get these devices onto operating heavy vehicles is a committed effort between the government, the tractor/trailer manufacturers, and the trucking fleets. The government has seen the opportunity to improve the fuel economy of heavy vehicles and has taken the initiative to support research and development in heavy vehicle aerodynamics. The involvement of tractor/trailer manufacturers can provide expertise in both road testing and design issues of these devices. The trucking fleets can give practical insight into how these devices impact the operational capability of their fleets and foresee any potential concerns. Only until this collaboration is established will the nation be able to benefit from the significant cost savings that these second-generation drag reduction devices can provide.

\section{$\underline{\text { References }}$}

${ }^{1}$ Cooper, K.R., “Truck Aerodynamics Reborn-Lessons from the Past," SAE Paper No. 2003-01-3376, 2003.

${ }^{2}$ Cooper, K.R., "The Effect of Front-Edge Rounding and Rear-Edge Shaping on the Aerodynamic Drag of Bluff Vehicles in Ground
Proximity," Paper No. 850288, SAE International Congress, Detroit, MI, February 25-March 1, 1985.

${ }^{3}$ Ingram, K.C., "The Wind-Averaged Drag Coefficient Applied to Heavy Goods Vehicles," Transport and Road Research Laboratory Supplementary Report 392, 1978.

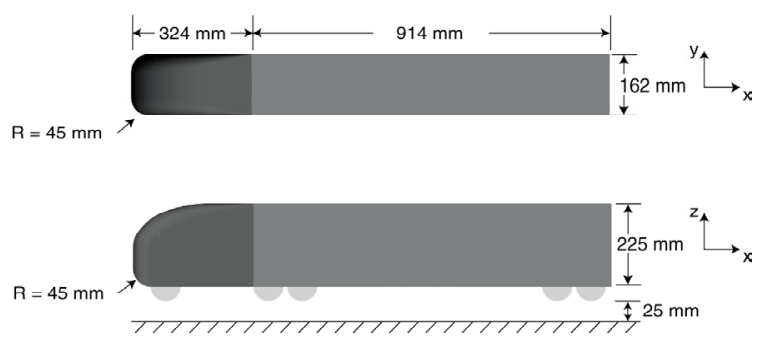

Figure 1. Model of the cab-over engine tractortrailer used in the wind tunnel study.

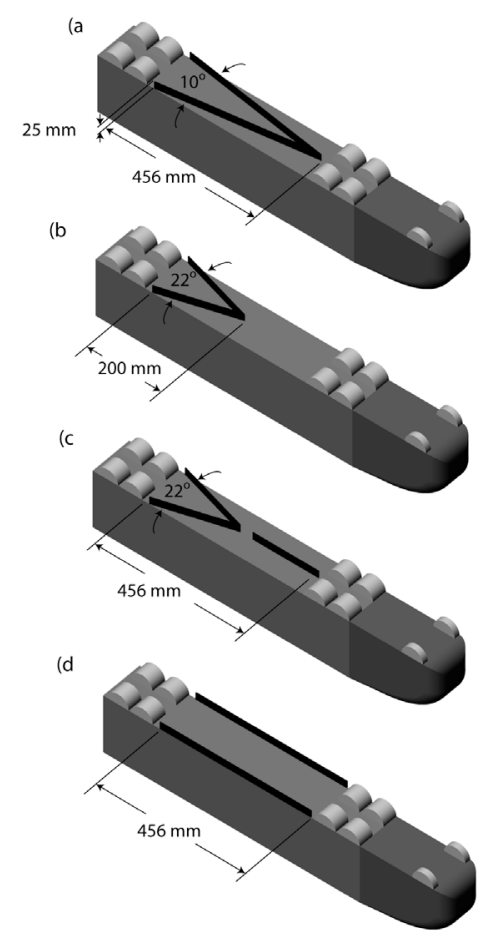

Figure 2. Skirt designs used to reduce the trailer underbody aerodynamic drag: a) long wedge skirt; b) short wedge skirt; c) short wedge skirt with center skirt; d) straight side skirts. (Note that the gap between the center skirt and short wedge skirt in c) is to allow clearance for the sting mount.) 

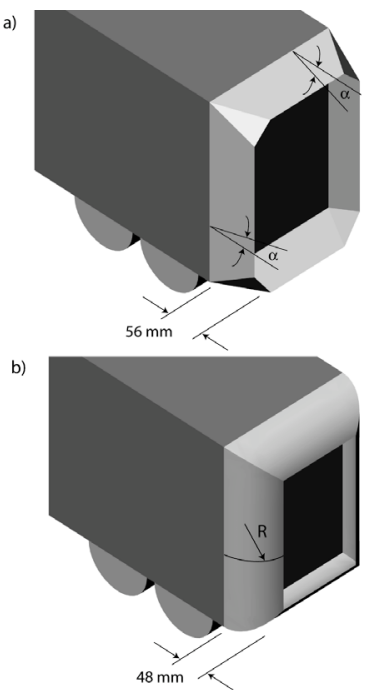

Figure 3. Base flap designs: a) angled base flaps; b) curved base flap 


\section{B. Investigation of Predictive Capability of RANS to Model Bluff Body Aerodynamics}

\section{Objective}

- Investigate the applicability of state-of-the-art computational modeling and simulations to predict the flow field around bluff bodies as related to the DOE Heavy Vehicle aerodynamics project. Specifically, determine the predictive capability of several commonly used, steady, Reynolds Averaged Navier-Stokes (RANS) turbulence models.

- Provide tractor and trailer manufacturers a knowledge base that describes the advantages and disadvantages of each turbulence model when selected for use within a commercial code.

\section{Approach}

- Perform simulations of a simplified tractor/trailer geometry for validating the drag prediction capabilities of selected RANS turbulence models.

- Compare the force coefficients, as well as the surface pressures and flow features, to experimental data ${ }^{1}$ of the Ground Transportation System (GTS) at $0^{\circ}$ and $10^{\circ}$ yaw conducted in conjunction with NASA Ames Research Center in their 7' 10' wind tunnel.

\section{Accomplishments}

- Comparison between the experimentally obtained floor boundary layer profile at the wind tunnel testsection entrance to those predicted by each of the turbulence models.

- Computed aerodynamic force coefficients for the selected turbulence models were compared to the experimental data at yaw angles of $0^{\circ}$ and $10^{\circ}$. Menter's two-equation BSL RANS model shows the closest agreement to the experimental data, generally predicting drag within 5\%. However, all of the models fail to capture the structure of the wake flow near the trailer base. Consequently, all of the selected models fail to reproduce the experimental base pressure coefficients.

\section{Future Direction}

- Investigate the applicability of unsteady RANS, large-eddy simulation (LES), and combinations of the two, for the prediction of these massively separated base flows.

\section{Introduction}

The objective is to investigate the applicability of state-of-the-art computational modeling and simulations to predict the flow field around bluff bodies as related to the DOE Heavy Vehicle aerodynamics project. Specifically, the goal is to determine the predictive capability of several commonly used, steady, Reynolds Averaged NavierStokes (RANS) turbulence models. This will provide tractor and trailer manufacturers a knowledge base that describes the advantages and disadvantages of each turbulence model choice.

\section{Computation Approach and Results}

Simulations are performed on a $1 / 8^{\text {th }}$ scale simplified tractor/trailer geometry for validating the drag prediction capabilities of selected RANS turbulence models. These models include the one-equation SpalartAllmaras (SA) ${ }^{2}$ and the two-equation Wilcox $(\mathrm{KW})^{3}$ and Menter (BSL) ${ }^{4} \mathrm{k}-\varepsilon$ models. The force coefficients, as well as the surface pressures and flow features, are compared to experimental data ${ }^{1}$ of the Ground Transportation System (GTS) at $0^{\circ}$ and $10^{\circ}$ yaw. This data was acquired from an experiment conducted in conjunction with 
NASA Ames Research Center in their $7^{\prime} 10^{\prime}$ wind tunnel.

Simulations of the GTS are run using NASA's OVERFLOW code. OVERFLOW is a fully compressible, 3-D, finite volume code employing overset grids. In all simulations, the flow is assumed to be fully turbulent and no attempt is made to model transition.

Additionally, no wall functions are used, as all turbulence equations are integrated to the wall.

As the GTS model is exposed to the floor boundary layer of the 7'_10' wind tunnel, the accuracy of a validation simulation depends on how well the upstream boundary layer is represented. Thus, a portion of the wind tunnel geometry is modeled, where careful attention is paid to matching the simulation boundary conditions to the actual wind tunnel conditions. Figure 1 presents a comparison between the experimentally obtained floor boundary layer profile at the wind tunnel testsection entrance to those predicted by each of the turbulence models.

Corresponding to the NASA experiment ${ }^{1}$ run 7, points 9 and 5, the GTS baseline configuration at $0^{\circ}$ and $10^{\circ}$ yaw are selected for simulation. Using the SA, KW, and BSL models, $0^{\circ}$ yaw simulations are conducted on a 14 million element grid. In addition, the BSL model is chosen to ensure grid convergence of the solution on a coarser 11 million element grid. In a similar fashion, $10^{\circ}$ yaw simulations are run on 19 and 14 million element meshes.

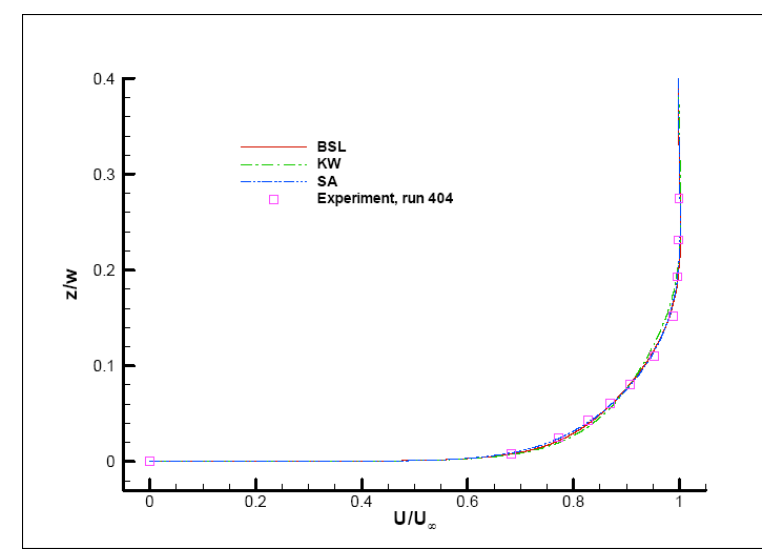

Figure 1. Floor Boundary Layer Velocity Profile

Tables 1 and 2 show the computed aerodynamic force coefficients for the selected turbulence models and the experimental data at yaw angles of $0^{\circ}$ and $10^{\circ}$ respectively. Among these models, Menter's two-equation BSL shows the closest agreement to the experimental data, generally predicting drag within $5 \%$.

\begin{tabular}{|l|c|c|c|}
\hline & CD & CL & CS \\
\hline SA, 14 million elements & 0.3173 & -0.1317 & $5.1 \mathrm{E}-06$ \\
\hline KW, 14 million elements & 0.2377 & -0.1070 & $-4.3 \mathrm{E}-04$ \\
\hline BSL, 14 million elements & 0.2638 & -0.1214 & $-1.4 \mathrm{E}-05$ \\
\hline BSL, 11 million elements & 0.2684 & -0.1220 & $-3.0 \mathrm{E}-06$ \\
\hline NASA experiment & $0.263 \pm 0.01$ & $-0.152 \pm 0.01$ & $0.007 \pm 0.01$ \\
\hline
\end{tabular}

Table 1. Aerodynamic Force Coefficients at $0^{\circ}$ Yaw

\begin{tabular}{|l|c|c|c|}
\hline & CD & CL & CS \\
\hline SA, 19 million elements & 0.6361 & -0.1162 & 1.1451 \\
\hline KW, 19 million elements & 0.5708 & -0.0236 & 1.1431 \\
\hline BSL, 19 million elements & 0.5626 & -0.0320 & 1.1376 \\
\hline BSL, 14 million elements & 0.5701 & -0.0252 & 1.1329 \\
\hline NASA experiment & $0.542 \pm 0.01$ & $0.026 \pm 0.01$ & $1.200 \pm 0.01$ \\
\hline
\end{tabular}

Table 2. Aerodynamic Force Coefficients at $10^{\circ}$ Yaw

Differences amongst the turbulence models in the computed force coefficients are found to be primarily due to contribution of the predicted pressure field around the GTS model. The computed pressure coefficients for each model are in close agreement with the experimental data with the exception of the 
area near the base of the trailer. Figures 2 and 3 illustrate the agreement between the predicted pressure coefficients and those obtained along the centerline of the top surface of GTS at $0^{\circ}$ and $10^{\circ}$ yaw. It is clear, however, that none of the turbulence models capture either the pressure field magnitude or trend at the base of the trailer. Figures 4 and 5 display the discrepancy between the computed pressure coefficients along the base centerline and the experimental data for each yaw angle.

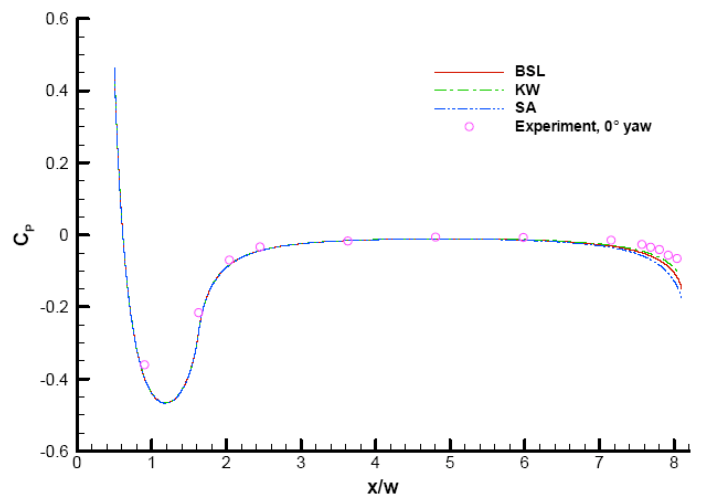

Figure 2. GTS Top Surface Centerline Pressure Coefficients at $0^{\circ}$ Yaw

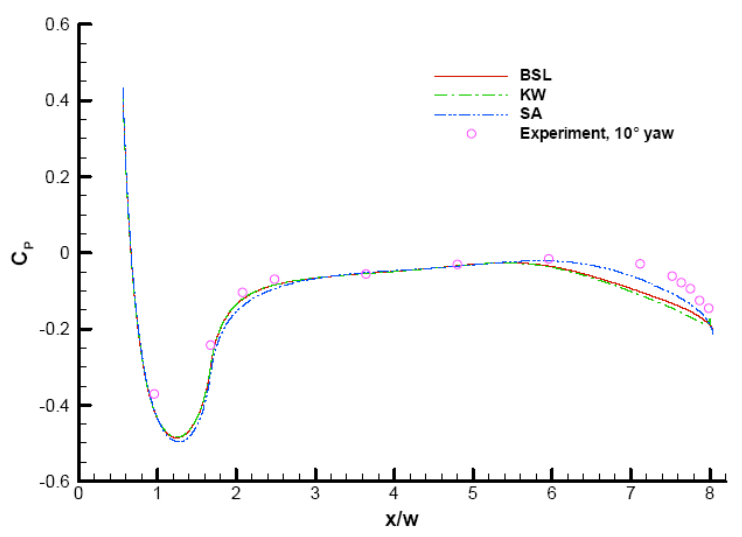

Figure 3. GTS Top Surface Centerline Pressure Coefficients for $10^{\circ}$ Yaw

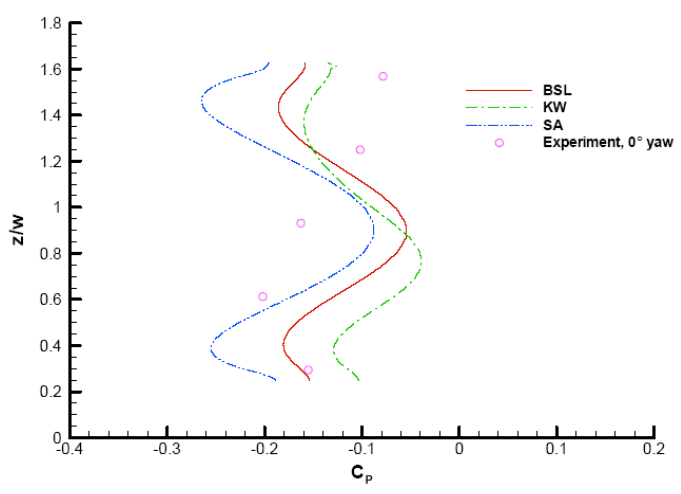

Figure 4. GTS Base Centerline Pressure Coefficients at $0^{\circ} \mathrm{Yaw}$

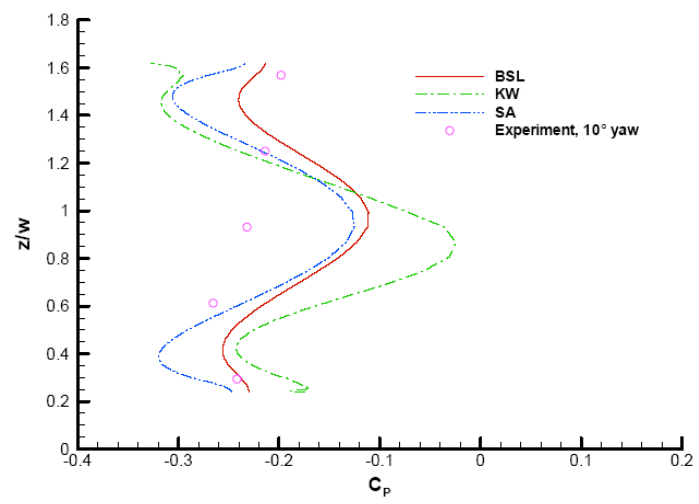

Figure 5. GTS Base Centerline Pressure Coefficients at $10^{\circ}$ Yaw

The accuracy of the base flow predicted by the selected turbulence models is further illustrated by examining the time-averaged PIV data available in the wake of the trailer. Although hidden by the similarity in force coefficients, the selected turbulence models predict very different separated wakes, particularly in the low velocity area very near the base of the trailer. As expected from the computed pressure coefficients, none of the models fully capture the separated flow structure indicated by the time-averaged PIV data. For example, along the model centerline, Figure 6 shows the BSL wake at $0^{\circ}$ yaw. For comparison, particle traces of the time-averaged PIV data is provided in Figure 7. 


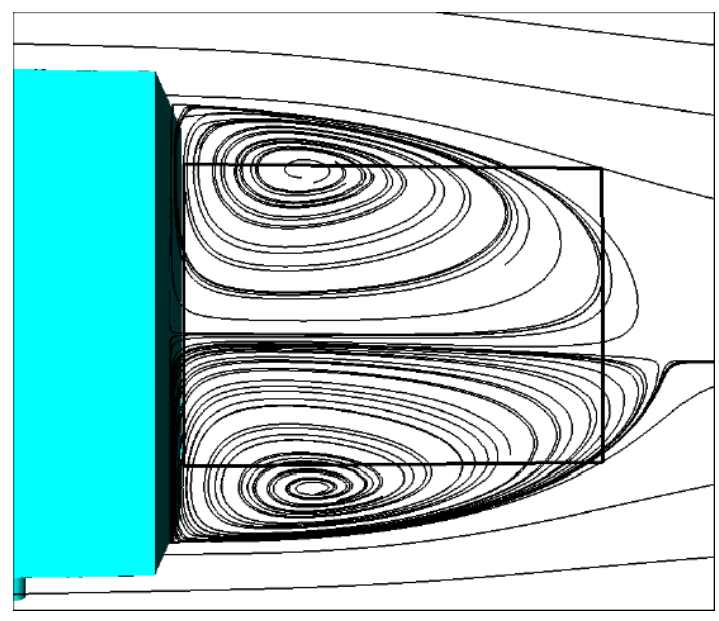

Figure 6. BSL Model Centerline Wake Flow Particle Traces

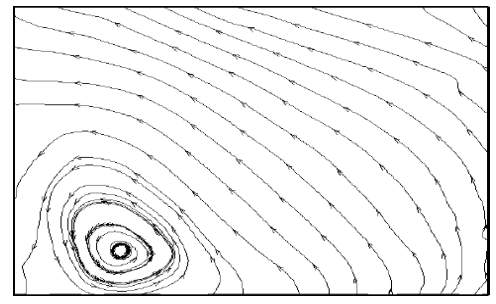

Figure 7. Particle Traces of Time-averaged PIV Data Within the Frame in Figure 6

\section{Conclusions}

Overall, the Menter BSL RANS model simulation is closer to experiment than either the KW or SA simulations, however, all of the models fail to capture the structure of the wake flow near the base. Consequently, all of the selected models fail to reproduce the experimental base pressure coefficients.
Although the drag force coefficients appear to be somewhat insensitive to the details of the flow near the base, to assess the efficacy of drag-reducing devices in the wake, steady RANS models are likely to prove to be inadequate. In order to consistently achieve drag values to within the incremental values produced by trailer base treatments, unsteady simulations may be required to fully represent the wake flow. Future plans call for investigation of the applicability of unsteady RANS, large-eddy simulation (LES), and combinations of the two, for the prediction of these massively separated base flows.

\section{References}

Storms, B., et. al., "An Experimental Study of the Ground Transportation System (GTS) Model in the NASA Ames 7- by 10-Ft Wind Tunnel,"NASA/TM-2001-209621, Feb. 2001.

2

Spalart, P.R. Allmaras, S.R., A One-Equation Turbulence Model for Aerodynamic Flows, AIAA Paper 92-439, Reno, NV, 1992.

${ }^{3}$ Wilcox, D.C., Turbulence Modeling for CFD, $2^{\text {nd }}$ Edition, DCW Industries, 1998.

${ }^{4}$ Menter, F.R., Two-Equation Eddy-Viscosity Turbulence Models for Engineering Applications, AIAA J., 32 (8), 1994, 15981605. 


\section{Splash and Spray Suppression}

\section{Objective}

- To analyze the formation and spread of spray clouds generated around and behind heavy vehicles using both laboratory experiments and validated computational models.

- Develop a collection of predictive computational tools available to tire and truck manufacturers that will allow the effects of add-on splash and spray suppression devices on vehicle aerodynamics and potential synergies between add-on aerodynamic devices and spray mitigation to be thoroughly explored.

\section{Approach}

- The droplet size, space, and velocity distributions determined as a function of tire speed and tread pattern from our experimental investigation will be used as an initial condition for our computational simulations.

- Commercially available computational fluid dynamics (CFD) codes will be used to predict the aerodynamic atomization of these droplets. User-defined atomization subroutines will be added to the commercial packages as necessary.

- Detailed computational investigations of the aerodynamics of rotating tires and wheel-wells and their effect on splash and spray will be performed.

- We will investigate the effects of splash and spray suppression devices on brake cooling and to explore possible enhancements to brake cooling resulting from well-designed add-on aerodynamic devices.

- We will employ validated state of the art large-eddy simulation (LES) models capable of capturing the spatial evolution of fine droplets in a turbulent flow that will be developed and used to simulate the dispersion of mist around and behind heavy trucks.

\section{Accomplishments}

- Reviewed the literature to determine both the existence and outcome of prior efforts toward splash and spray suppression and to delineate the pertinent physics governing the problem.

- Coordinated experimental research plan with team at USC.

- Working with USC, an experimental test rig was developed.

- Began investigating STAR-CD's ability to track free fluid surfaces via its implementation of the volume of fluid (VOF) method and to predict ensuing droplet formation.

- Began studying STAR-CD's ability to accurately simulate the flow around a rotating tire, including both aerodynamic and free-surface phenomena.

- Began investigating STAR-CD's ability to capture aerodynamic droplet breakup (secondary atomization) and turbulent transport of sub-Kolmogorov scale particles.

\section{Future Direction}

- This project is in its very nascent stages. All aspects of the project discussed above are under continuing development.

\section{Introduction}

The spray clouds generated around and behind heavy trucks traveling on highways during adverse weather conditions lead to reduced visibility and increased anxiety for motorists traveling nearby and pose a significant public safety concern. Our objective is to analyze the formation and spread of these clouds using both laboratory 
experiments and validated computational models.

\section{Experiments}

Fred Browand and co-workers at the University of Southern California have developed a unique test rig to investigate water pick-up by tire treads and the primary formation of droplets in the wake of a rotating tire. The test rig consists of a tubular aluminum frame supporting two spinning wheels mounted pendulum-style upon swinging rigid arms. Commercially available, thirteen-inch diameter wheels are used. Michelin of North America is providing custom tires with specified tread designs. The two wheels are pressed together with a lateral spring-damper to form a well-defined, accurately controllable tire patch. The wheels are rolled in contact with one another using a sprocket and chain drive. The plane of symmetry of the experimental facility replaces the road surface. Water is introduced from a solenoid-actuated injector placed just ahead of the tire patch and is injected at a velocity matching the peripheral speed of the tires, as would be the case for a rolling tire on a wet road.

The formation of water droplets just downstream of the contact patch is studied using a digital camera, pulsed laser light, and numerical algorithms similar to those developed for digital particle image velocimetry (DPIV). High-speed (4,000 $16,000 \mathrm{fps}$ ) digital images of ligament formation and droplet ejection from the tire surface are also feasible. This facility allows the investigation of various tread geometries to understand their function in water uptake and primary droplet formation in a much more controlled manner than has hitherto been possible.

\section{Computations}

The droplet size, space, and velocity distributions determined as a function of tire speed and tread pattern from our experimental investigation will be used as an initial condition for our computational simulations. Commercially available computational fluid dynamics (CFD) codes will be used to predict the aerodynamic atomization of these droplets. User-defined atomization subroutines will be added to the commercial packages as necessary. The aerodynamics of rotating tires and wheel-wells are of paramount importance to the secondary atomization process.

Detailed computational investigations of these effects will be performed in conjunction with the atomization study. This will allow us to simultaneously investigate the effects of splash and spray suppression devices on brake cooling and to explore possible enhancements to brake cooling resulting from well-designed add-on aerodynamic devices.

Validated state of the art large-eddy simulation (LES) models capable of capturing the spatial evolution of fine droplets in a turbulent flow will be developed and used to simulate the dispersion of mist around and behind heavy trucks. Ultimately, we wish to develop a collection of predictive computational tools available to tire and truck manufacturers. It is desired, therefore, to implement the specialized models developed in this phase of the study as user subroutines in widely available CFD codes. The result will be a package that allows the effects of add-on splash and spray suppression devices on vehicle aerodynamics and potential synergies between add-on aerodynamic devices and spray mitigation to be thoroughly explored.

\section{Accomplishments and Conclusions}

We began this study with an extensive review of the literature to determine both the existence and outcome of prior efforts toward splash and spray suppression and to delineate the pertinent physics governing the problem. We helped to coordinate the experimental research plan with the team at USC and develop the experimental test rig. We began studying the suitability of commercial CFD codes to (1) simulate both primary and secondary formation of droplets and (2) to simulate water entrainment and ejection by 
rotating tires. In particular, we have begun investigating STAR-CD's ability to track free fluid surfaces via its implementation of the volume of fluid (VOF) method and to predict droplet formation. We have also begun studying STAR-CD's ability to accurately simulate the flow around a rotating tire, including both aerodynamic and free-surface phenomena. We have also begun investigating STAR-CD's ability to capture aerodynamic droplet breakup (secondary atomization) and turbulent transport of sub-Kolmogorov scale particles.

\section{Future Direction}

This project is in its very nascent stages. All aspects of the project discussed above are under continuing development. 


\section{Computational Investigation of Aerodynamics of Rail Coal Cars and Drag Reducing Add-On Devices}

\section{Objective}

- To reduce the aerodynamic drag of an empty rail coal car through use of drag reducing add-on devices.

\section{Approach}

- The turbulent time-averaged flow field around a generic coal car at realistic Reynolds number is simulated using RANS computational approach.

- Design drag reducing add-on devices and test using the same computational modeling technique.

\section{Accomplishments}

- The turbulent flow field around the coal car was computed by Star-CD using standard high Reynolds number k- $\varepsilon$ turbulence model with wall function. A large recirculation area within the interior of the car that extends over almost the entire length of the car is largely responsible for producing the high aerodynamic drag for an empty coal car.

- Designed a new add-on device to manipulate the interior flow structure of the car. Computations indicate a $10 \%$ drag reduction over the base empty coal car with this new device.

\section{Future Direction}

- Further investigate the potential of LLNL drag reducing add-on device by optimizing the number, the location and the height of the plates.

- Collaborate with NASA Ames on their experimental study of coal car aerodynamics and drag reducing add-on devices

\section{Introduction}

To reduce the aerodynamic drag of an empty rail coal car through use of drag reducing add-on devices. Constraints are imposed on the design of these devices such as: no interference with loading and unloading, no alteration of car internal volume, and no or minimum maintenance.

The turbulent time-averaged flow field around a generic coal car at realistic Reynolds number is simulated using the RANS computational approach. The predicated flow field is carefully investigated for possible flow structures that are susceptible to manipulation by add-on devices. Then, drag reducing addon devices are designed and tested using the same computational modeling technique.

\section{Computations and Results}

To perform modeling and simulation on an empty coal, car a generic model was needed. A CAD definition of such a model was provided by Jim Ross at NASA Ames for this investigation. NASA is currently studying the aerodynamics of loaded and unloaded coal cars through a series of experiments. As the NASA experimental data becomes available the computational results can be validated. The geometry of the generic coal car is shown in Fig. 1 with dimensions of $6.84 \mathrm{~m} \mathrm{x} 1.45 \mathrm{~m} \mathrm{x}$ $1.80 \mathrm{~m}$.

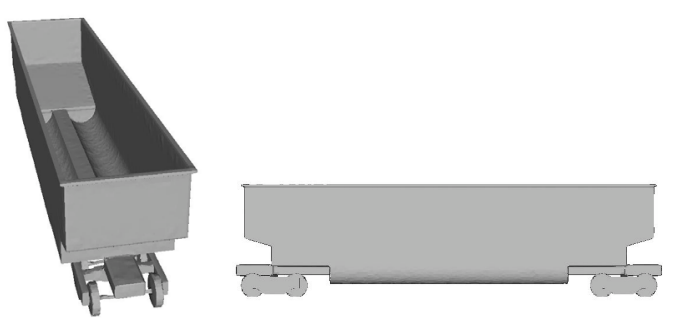

Figure 1. Generic coal car geometry

The turbulent flow field around the coal car was computed by Star-CD using standard high Reynolds number k- $\varepsilon$ turbulence model with wall function. The flow is assumed to be incompressible and fully turbulent. A realistic flow Reynolds number of 4 million based on 
the car width and flow velocity of $70 \mathrm{~km} / \mathrm{hr}$ (43.6 miles/hr) were used for all computations. Figure 2 presents the flow field around the empty coal car using particle traces. The stagnation area in front and flow over the car is clearly visible. Figure 3 shows a large recirculation area within the interior of the car that extends over almost the entire length of the car. This flow structure is largely responsible for producing the high aerodynamic drag for empty coal car. The computed drag coefficient for this case is 1.28 and the lift coefficient is -0.1 (downward force).

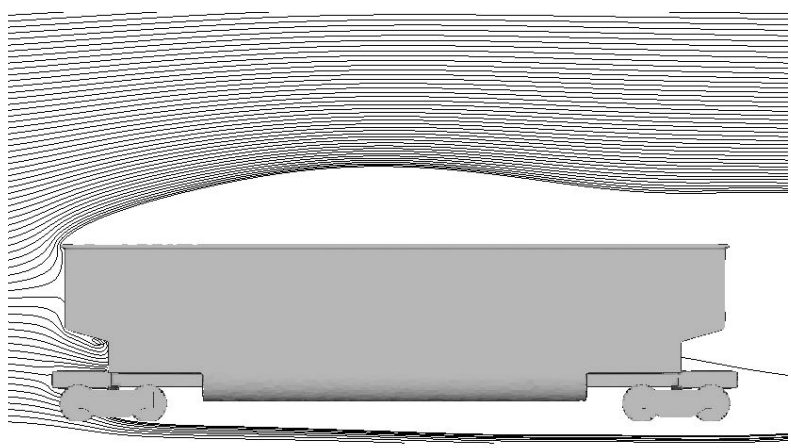

Figure 2. Particle traces showing the flow field around the coal car

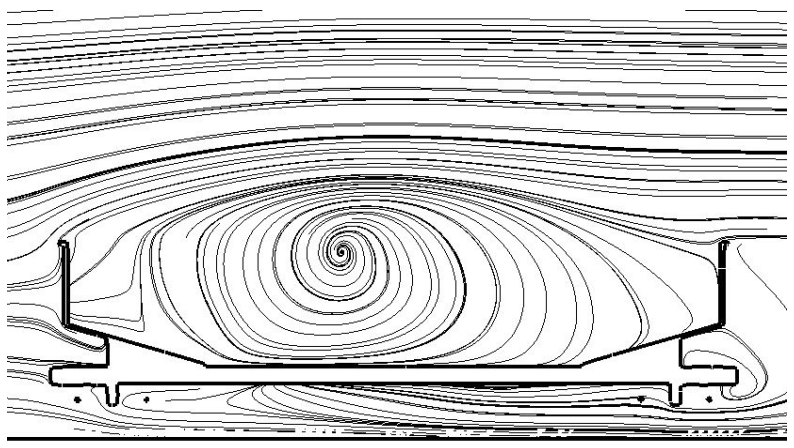

Figure 3. Particle traces on a symmetry plane including the interior space

To reduce the aerodynamic drag of this generic coal car, the flow recirculation within the car needs to be modified. Figure 4 presents a new LLNL add-on device designed to manipulate the interior flow structure of the car. Presently, the device consists of two thin plates that split the car in $1 / 3$ section and they extend half way up as shown in Fig 4. The number of plates could range from 1 to 10 and the height of each plate could vary from a $1 / 3$ up to the full height of the car. The device is extremely simple and should not require any maintenance. Due to utilization of thin plates, the coal car capacity and loading and unloading are not affected. This is our first attempt to evaluate the performance of LLNL drag reducing device.
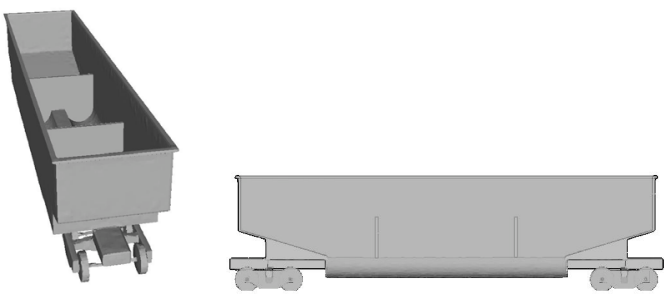

Figure 4. Generic coal car with the aerodynamic add-on device

Figures 5 and 6 present the computed flow field around the coal car caused by the presence of the aerodynamic add-on devices. Figure 5 shows a similar flow pattern to Fig 2 with the exception that particle traces show lower flow deflection on top of the car. The reason for this is shown in Fig 6 where the flow recirculation is confined to a region between the two thin plates. For this geometry the drag and lift coefficients are 1.16 and 0.15 , respectively. This represents a $10 \%$ drag reduction over the base empty coal car. Due to the limited scope of this effort no attempt has been made to optimize the number, spacing, and the height of the plates for maximum possible drag reduction.

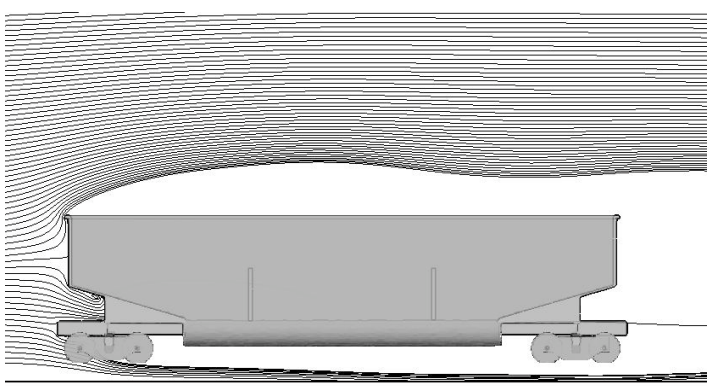

Figure 5. Particle trace showing the flow field about the coal car with the add-on device 


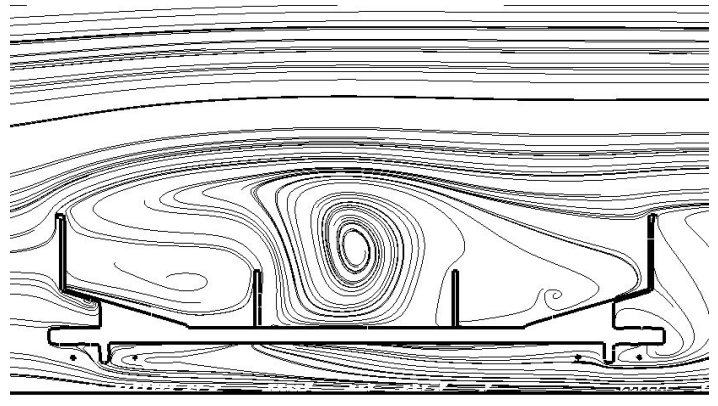

Figure 6. Particle traces on a symmetry plane including the interior space with the add-on device

\section{Conclusions}

Further investigate the potential of LLNL drag-reducing add-on device by optimizing the number, the location and the height of the plates. Also, collaborate with NASA Ames with their experimental study of coal car aerodynamics and drag-reducing add-on devices 


\title{
APPENDIX E
}

\section{Computational/Analytical Support; SANDIA National Laboratories: Computational and Analytical Simulation of Simplified GTS Geometries/Bluff Bodies}

\author{
Principal Investigator: Lawrence J. De Chant (PI), Basil Hassan, Jeff Payne, Matt Barone, Chris \\ Roy (Contractor: Auburn U.) \\ Affiliation: Sandia NationalLaboratory \\ Address: P.O. Box 5800, Albuquerque, NM 87185-0825 \\ Phone, Fax, E-Mail: 505-844-4250, 505-844-4523 (fax) ljdecha@sandia.gov
}

Technology Development Area Specialist: Sidney Diamond

(202) 586-8032; fax: (202) 586-1600; e-mail: sid.diamond@ee.doe.gov

Technical Program Manager: Jules Routbort

(630)252-5065; fax: (630)252-4289; email: routbort@anl.gov

Contractor: Sandia National Laboratories

Contract No.: DE-AC04-94AL85000

\section{Objective:}

- Examine the effectiveness of the Detached Eddy Simulation (DES) approach for predicting tractor/trailer wakes

- Explore potential for reduced order drag coefficient model based upon combined Green's function and GramCharlier series method

\section{Approach:}

- Sandia's SACCARA code was used to conduct DES simulations of a truncated version of the Ground Transportation System (GTS) without boattail plates

- Preliminary computations were run on a coarse (4 million cell) mesh

- The DES simulations were compared to steady-state Reynold's-Averaged Navier-Stokes (RANS) computations and experimental data

- A reduced-order model was developed using self-similar, far-field, turbulent wake concepts to estimate the 2-d drag coefficient for a range of bluff body problems

\section{Accomplishments:}

- Statistically converged unsteady DES solutions were obtained for the truncated GTS model on the coarse mesh at a Reynolds number of 2 million

- The DES predictions were compared to steady-state RANS simulations using the Menter k-omega model

- The DES predictions were compared to experimental data obtained in the NASA-Ames 7'x10' wind tunnel

- Comparisons include both time-averaged surface pressure and time-averaged wake velocities

- A reduced-order model was developed using self-similar, far-field, turbulent wake concepts to estimate the 2-d drag coefficient for a range of bluff body problems

\section{Future Direction:}

- Initial estimates suggest that the Sandia funding levels will be significantly reduced from prior levels

- Auburn University will examine the DES simulations of the full GTS geometry using finer meshes 


\section{A. DES Simulations}

\section{Introduction:}

Preliminary results are presented for Detached Eddy Simulations (DES) of a generic tractor/trailer geometry at a Reynolds number of 2 million based on the trailer width. The DES simulations are compared to both experimental data and to steady-state Reynolds-Averaged Navier-Stokes (RANS) computations using the Menter k-w turbulence model. These comparisons include both time-averaged base pressures and wake velocities. The DES results with the truncated geometry do not provide improved agreement with the experimental data relative to the steady-state RANS results with the full geometry. The lack of improved agreement may be due to either insufficient mesh refinement or the use of the truncated geometry. Further details of this work are presented in Ref. 1.

\section{Approach:}

The computational fluid dynamics code used herein is SACCARA, the Sandia Advanced Code for Compressible Aerothermodynamics Research and Analysis. The SACCARA code was developed from a parallel distributed memory version of the INCA code, originally written by Amtec Engineering. The SACCARA code is used to solve the Navier-Stokes equations for conservation of mass, momentum, energy, and turbulence transport in either 2D or 3D form. Prior code verification studies with SACCARA include code-to-code comparisons with other NavierStokes codes and with the Direct Simulation Monte Carlo method. These studies provide some confidence that the code is free from coding errors affecting the discretization.

For the simulations results presented herein, the turbulence transport equations are integrated all the way to the vehicle walls, thus no wall functions are employed. In all cases, the distance from the wall to the first cell center off the wall is less than unity in normalized turbulence distance (i.e., $\mathrm{y}^{+<1}$ ). The steady-state RANS model examined is Menter's hybrid model which switches from a k-epsilon formulation in the outer flow to a k-omega formulation near solid walls.
The hybrid RANS/LES method developed by Spalart and co-workers has been developed the furthest and is called Detached Eddy Simulation, or DES. The DES approach uses the unsteady form of the Spalart-Allmaras one-equation eddy viscosity model to provide the eddy viscosity for use in the sub-grid scale stress model. The Spalart and Allmaras one-equation eddy viscosity model provides the usual RANS-based eddy viscosity in the boundary layer, but must be modified to the appropriate eddy viscosity for LES outside of the boundary layer.

The flow over the Ground Transportation System (GTS) model has been investigated experimentally at a Reynolds number $\mathrm{Re}_{\mathrm{W}}$ of 2 million by Storms et al [Ref. 2], where $\mathrm{W}$ refers to the width of the trailer base $(0.32385 \mathrm{~m})$. The experimental data set is unique in that it presents both ensemble-averaged surface pressure data as well as multiple planes of instantaneous and ensemble-averaged velocity data in the wake for this high Reynolds number flow.

In order to perform the computationally intensive DES simulations, the front of the GTS was truncated along with the wind tunnel wall at $\mathrm{x} / \mathrm{W}=2$. Note that the wind tunnel wall as well as the rear posts are included in the simulation. This mesh is composed of approximately 4 million grid points and was domain decomposed and run on 32 processors of a Linux cluster.

\section{Accomplishments:}

Preliminary results were presented for Detached Eddy Simulations (DES) of generic tractor/trailer geometry at a Reynolds number of 2 million based on the trailer width for the truncated geometry. The DES simulations were compared to both experimental data and to steady-state Reynolds-Averaged Navier-Stokes (RANS) computations using the Menter k-omega turbulence model. These comparisons included both time-averaged base pressures and wake velocities. Although we fully expected the DES simulations to improve the agreement with the experimental data, the results were clearly not better than the steady-state RANS computations (see Fig. 1). 
The fact that the DES simulations did not provide improved agreement with the experimental data as compared to the RANS results could be due to a number of reasons. The residuals for each time step did not obtain the desired iterative convergence level due to the extremely fine mesh spacing near the walls, thus some iterative errors may be polluting the solution. The effects of truncating the forward part of the GTS geometry and the wind tunnel were not assessed. The flow over the forward portion of the GTS does generate some streamwise vorticity which is not included in the current simulations.

The back pressure was adjusted, and then run for $0.1 \mathrm{~s}$ before statistics were collected.

Examination of the reference pressure indicated that the pressure transients had not yet died out by the time statistics were collected. In addition, the time window for collecting statistics should be increased to ensure the time-averaged results are statistically converged. Finally, another possible reason for the lack of agreement between the DES simulations and the experiment is insufficient mesh refinement. Comparison of the unsteady pressure signal from the experiment with the same signal from the DES simulations showed that the DES signal had less structure and a larger amplitude. This behavior is likely related to the excessive dissipative errors associated with insufficient mesh refinement.

\section{References:}

1. C. J. Roy, J. C. Brown, L. J. DeChant, and M. A. Barone, "Unsteady Turbulent Flow Simulations of the Base of a Generic Tractor/Trailer," AIAA Paper 2004-2255, 34th AIAA Fluid Dynamics Meeting, Portland, OR, June 2004.

2. B. Storms, Ross JC, Heineck JT, Walker SM, Driver DM, Zilliac GG, "An Experimental Study of the Ground Transportation System (GTS) Model in the NASA Ames 7- by 10 -ft Wind Tunnel," NASA TM-2001-209621, 2001.

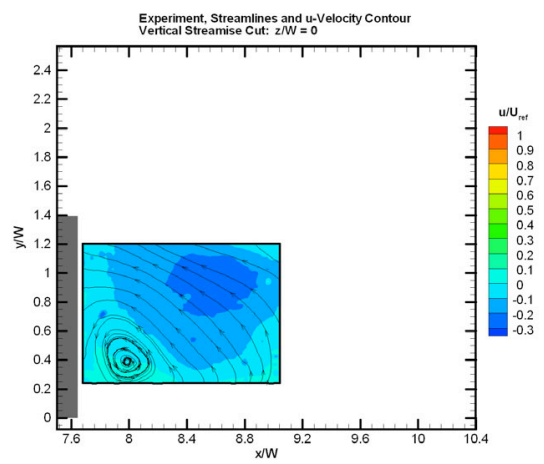

a) experiment

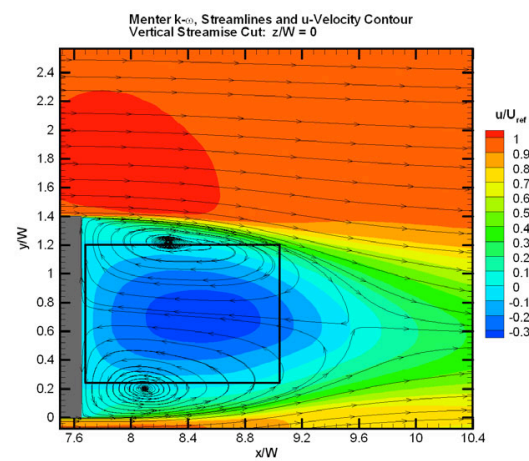

b) RANS

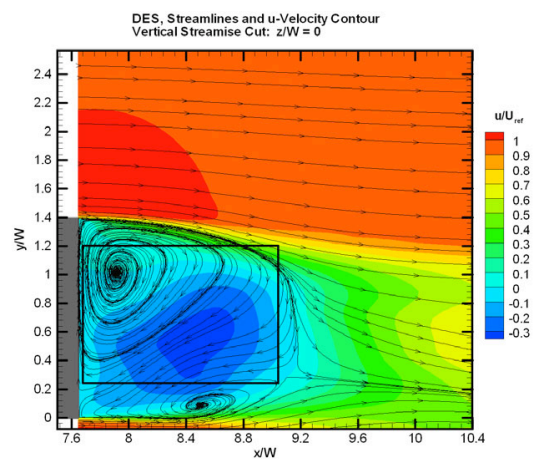

c) $\mathrm{DES}$

Fig. 1: Vertical streamwise cuts of u-velocity and streamlines from a) experiment, b) RANS, and c) DES. 


\section{B. Reduced-Order Model}

\section{Introduction}

In this study, we extend self-similar, far-field, turbulent wake concepts to estimate the 2-d drag coefficient for a range of bluff body problems. The self-similar wake velocity defect that is normally independent of the near field wake (and hence body geometry) is modified using a combined approximate Green's function/GramCharlier series approach to retain the body geometry information. Formally a near field velocity defect profile is created using small disturbance theory and the inviscid flow field associated with the body of interest. The defect solution is then used as an initial condition in the approximate Green's function solution. Finally, the Green's function solution is matched to the Gram-Charlier series yielding profiles that are integrated to yield the net form drag on the bluff body. Preliminary results indicate that drag estimates computed using this method are within approximately $15 \%$ as compared to published values for flows with large separation. This methodology may be of use as a supplement to CFD and experimental solutions in reducing the heavy computational and experimental burden of estimating drag coefficients for blunt body flows for preliminary design type studies.

Drag estimates for strongly separated flow over blunt bodies is an essential piece of information for many engineering systems. An application that demands our particular attention is aerodynamic drag forces on large ground transportation vehicles, i.e. tractor/trailer trucks. As noted by Roy et. al (2003) a common tractor/trailer, energy losses due to rolling resistance and accessories increase linearly with vehicle speed, while energy losses due to aerodynamic drag increase with the cube of the speed. At a typical highway speed of $70 \mathrm{mph}$, aerodynamic drag accounts for approximately $65 \%$ of the energy output of the engine (McCallen et al 1999). Due to the large number of tractor/trailers on the US highways, even modest reductions in aerodynamic drag can significantly reduce domestic fuel consumption. Lower fuel consumption will result in a reduction in pollution emissions and, significantly, a reduced dependence on foreign oil.

Though most modern computationally based aerodynamic drag reduction studies have focused on Computational Fluid Dynamics (CFD) methods utilizing evermore sophisticated (and concurrently computationally expensive) methodologies, there remains a valuable role for reduced complexity, analytically based models. Here we describe a model that provides an alternative to computationally expensive models.

\section{2-d Bluff Body Drag}

The basic methodology recalls that there must be a self-preservation solution (Tennekes and Lumley, 1972), e.g. self-similar construction for the velocity field, i.e.:

$$
\frac{U_{0}-U}{U_{s}}=f\left(\frac{y}{l}\right)
$$

is necessary where $U_{s}$ is the maximum crossstream variation in $U$. Note, that for wakes $U_{s}$ will be $\mathrm{U}(\mathrm{y}=0)$ where the relevant coordinate system and associated definitions are shown in figure 1 .

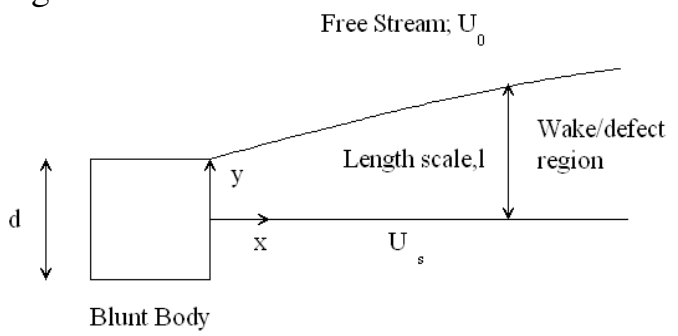

Figure 1 Schematic diagram of wake flow with coordinate, velocity and length scale definitions.

Unfortunately, the solution obtained by Tennekes and Lumley, cannot be strictly valid in the near field since the form of the similarity solution chosen by Tennekes and Lumley (1972) requires that $U_{s}=A x^{-1 / 2} \quad l=B x^{1 / 2}$ and $\mathrm{A}$ and $\mathrm{B}$ are constants to be determined. Obviously, the $\mathrm{U}_{\mathrm{s}}$ solution is not (and cannot be) valid for $\mathrm{x}<<1$. This limitation poses no problem in the far-field, of course, and is acceptable in an intermediate overlap region as well, but cannot be applied in the wake near-field. 
The connection to the velocity defect velocity field and the drag coefficient is given by (2-d drag coefficient); equation:

$$
C_{D}=2 \frac{\theta}{d}=\int_{-\infty}^{\infty} f d \eta
$$

The 2-d momentum thickness $\theta$ is introduced through equation (2) as well.

\section{$\underline{\text { Analysis }}$}

The classic, self-similar far field wake solution is not valid in the near field of the bluff body. However, by carefully examining the process used to derive the similarity solution, it is possible to achieve other formulations that are valid in the near field.

Approximate Green's function solution

An alternative approach to the physically based similarity arguments presented in the text involves the mathematical analysis of a generalized problem. Here we discuss a range of fundamental solutions to the heat equation (the canonical form of the linearized wake relationship). If, however we are willing to use the functional form of equation (9) we can form a solution to the governing equations that is selfsimilar, i.e.

$f_{G F}(\eta)=\frac{1}{\sqrt{\pi}} \int_{0}^{\infty} f_{\text {near }}(\bar{\eta})\left[\begin{array}{l}\exp \left(-(\eta+\bar{\eta})^{2}\right)+ \\ \exp \left(-(\eta-\bar{\eta})^{2}\right)\end{array}\right] d \bar{\eta}$

where $\eta_{0}$ denotes the dimensionless length scale associated with the bluff body. Note, that equation (3) will approximately satisfy the full range of conditions required for solution of the wake problem.

"Initial Condition Velocity Field"; Connection to the Inviscid Flow Field

To utilize equation (2) to obtain the defect velocity field, it is necessary to be able to compute a near field velocity defect profile, i.e. $f_{\text {near }}$. This function must provide the sum total of the bluff bodies geometric information. In terms of a practical result, it also must be readily obtainable and unique. Perhaps the most obvious closure for the near field defect solution that satisfies these requirements is to utilize the local inviscid potential flow solution.

For sharp edged bluff bodies, such as, the square cylinder shown in figure 1 . where the separation location is well established, the defect velocity field is readily estimated by the discontinuous step function:

$$
f_{\text {near }}=\left\{\begin{array}{cc}
1 & 0 \leq \eta \leq 1 \\
0 & \text { otherwise }
\end{array} ; \text { where } \eta=2 \mathrm{y} / \mathrm{d}\right. \text {. }
$$

Substitution of this near-field relationship into equation yields: $f_{G F}=\frac{\lfloor\operatorname{erf}(\eta+1)-\operatorname{erf}(\eta-1)\rfloor}{2}$. Notice, that when integrated the near field and Green's function solution, give

$\mathrm{C}_{\mathrm{D}=} \int_{-\infty}^{\infty} f_{\text {near }} d \eta=\int_{-\infty}^{\infty} f d \eta=2$. Though this estimate for 2-d drag is quite good, since $C_{D}$, exper=2.1 (White 1986), we note that the Green's function does not modify the net initial defect velocity, hence in terms of the drag coefficient the Green's function relationship provides no new information. Our reason to utilize the Green's function form will become apparent later, but we already note, that the Green's function relationship will satisfy several essential properties including:

1. continuous, differentiable flow field valid over full domain, i.e. $-\infty \rightarrow+\infty$.

2. satisfies symmetry and far field boundary conditions

3. and, approximately, satisfies governing linearized, momentum (diffusion) equation.

\section{Gram-Charlier Series}

In the previous section, it was clear that it is the local velocity defect solution that provides an estimate of drag since the Green's function relationship, equation (2), preserves the area under the local velocity defect approximation.

However, the classical far-field wake profiles derived by Tennekes and Lumley (1972) should be taken into account in any formulation.

We can achieve a far-field wake influence by noting that the far field wake might represent a single first term in a Gram-Charlier series 


$$
\begin{aligned}
& f(\xi)=a_{0} \exp \left(-\frac{1}{2} \eta^{2}\right)-a_{1} \xi\left[\exp \left(-\frac{1}{2} \eta^{2}\right)\right] \\
& -a_{2}\left(1-\eta^{2}\right)\left[\exp \left(-\frac{1}{2} \eta^{2}\right)\right]+\ldots
\end{aligned}
$$

Computing derivatives, evaluating and collecting terms, we write:

$$
\begin{aligned}
& f_{G F}(0)=a_{0}-a_{2} \\
& f^{\prime}{ }_{G F}(0)=-a_{1} \\
& f^{\prime \prime}{ }_{G F}(0)=3 a_{2}-a_{1}-a_{0}
\end{aligned}
$$

Notice that for symmetric problems (the possibility of asymmetry is included in the complete series) that we can write

$a_{0}=\frac{1}{2}\left(3 f_{G F}(0)-f^{\prime \prime}{ }_{G F}(0)\right)$.

\begin{tabular}{|l|l|l|l|l|}
\hline Shape & $\begin{array}{l}\text { Theor } \\
. \mathrm{C}_{\mathrm{D}}\end{array}$ & $\begin{array}{l}\text { Pub. } \\
\mathrm{C}_{\mathrm{D}}\end{array}$ & Reynolds \# & $\begin{array}{l}\text { Rel. } \\
\text { Error } \\
(\%)\end{array}$ \\
\hline $\begin{array}{l}\text { Square Cylinder } \\
\text { (step function) }\end{array}$ & 2.1 & 2.1 & Independent & $0 \%$ \\
\hline $\begin{array}{l}2: 1 \quad \text { Rectangular } \\
\text { Cylinder } \\
\text { (num. inviscid) }\end{array}$ & 1.85 & 1.7 & $\begin{array}{l}\text { Independent } \\
\left(\text { Re }>10^{3}\right)\end{array}$ & $10 \%$ \\
\hline $\begin{array}{l}\text { Equilateral (apex } \\
\text { triangle } \\
\text { facing flow) }\end{array}$ & 1.35 & 1.6 & Independent & $15 \%$ \\
\hline $\begin{array}{l}\text { Circular Cylinder } \\
\text { (laminar B. L.) }\end{array}$ & 1.3 & 1.2 & $10^{3}<\operatorname{Re}<10^{5}$ & $8 \%$ \\
\hline $\begin{array}{l}\text { Circular Cylinder } \\
\text { (Transition B. L.) }\end{array}$ & 0.63 & 0.6 & $\mathrm{Re} \approx 5 \mathrm{x} 10^{5}$ & $5 \%$ \\
\hline $\begin{array}{l}\text { Circular Cylinder } \\
\text { (Turbulent B. } \\
\text { L.) }\end{array}$ & 0.51 & 0.3 & $\mathrm{Re}>10^{6}$ & $70 \%$ \\
\hline $\begin{array}{l}\text { Parabolic } \\
\text { Cylinder, f=x(1-x) } \\
\text { (Small } \\
\text { disturbance)(Turb } \\
\text { ulent B. L.) }\end{array}$ & 0.15 & 0.2 & $\mathrm{Re}>>>1$ & $25 \%$ \\
\hline
\end{tabular}

Table 1 Preliminary comparison between published 2-d drag coefficient results and the theoretically-based model developed here. Notice that the theoretical model compares adequately with published values for rapidly separated flows (sharp edged and laminar) but performs poorly for smooth bodies with delayed separation.

\section{$\underline{\text { Results }}$}

Preliminary drag coefficient results for a range of 2-d bluff body shapes are presented in table 1 . Comparison is made with published values given in White (1986) and Hughes and Brighton (1991).

Though our interest is primarily focused upon deriving an estimate for the integrated value $C_{D}$, we can also utilize the preceding analysis to obtain estimates of the flow field behavior. Following Tennekes and Lumley (1972), and utilizing their variables $U_{s}=A x^{-1 / 2} \quad l=B x^{1 / 2}$ and $\mathrm{A}$ and $\mathrm{B}$ are constants to be determined we obtain an approximation for the centerline velocity behavior as a function of $\mathrm{x}: U_{s}=3.16 U_{0} d x^{-1}$. Of course, this expression is not valid for $\mathrm{x}<<1$, but we expect that the functional form, i.e. power of $\mathrm{x},-1$ to be correct. By way of comparison, we consider the centerline velocity data for flow over a square cylinder square cylinder given by Lyn et. al.

(1995) in figure 2.

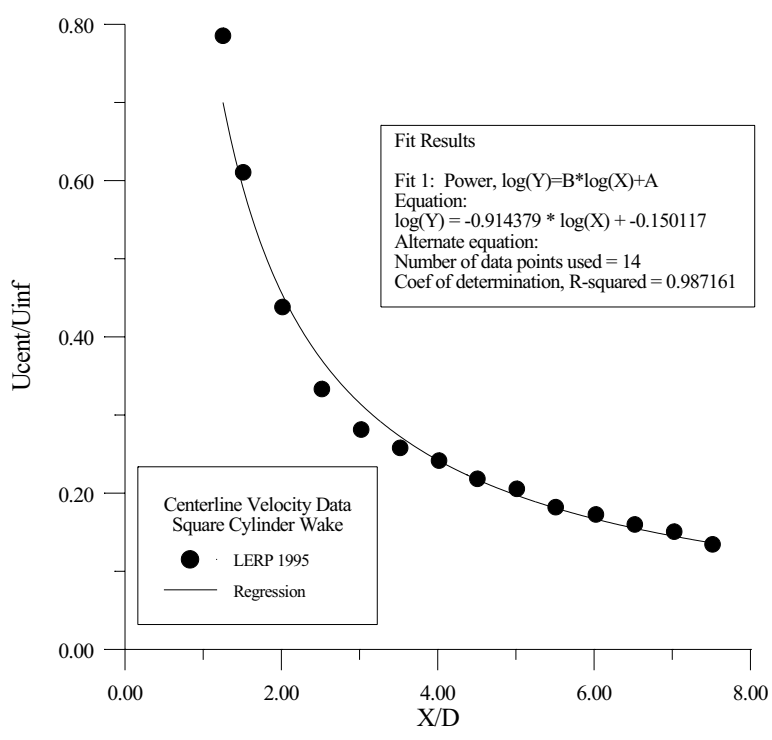

Figure 2. Centerline velocity data for flow over a square cylinder square cylinder given by Lyn et. al. (1995) with regression analysis. Notice that the curve-fit expression decays to the -0.91 power a value that compares well with the theoretical value, -1 .

\section{Conclusions}

In this manuscript we have modified classical self-similar, far-field, turbulent wake concepts to estimate the 2-d drag coefficient for a range of 
bluff body problems. Preliminary results indicate that drag estimates computed using this method are within approximately $10-20 \%$ as compared to published values for flows with large separation. The potential value of this method is as a way to utilize poorly resolved simulation results to provide an inexpensive estimate of body drag or as the basis of a physically consistent correlation scheme. This methodology may be of use as a supplement to CFD and experimental solutions in reducing the heavy computational and experimental burden of estimating drag coefficients for blunt body flows for preliminary design type studies.

\section{$\underline{\text { References }}$}

(1) Lyn, D. A, Einav, S., Rodi, W. and Park, J-H, 1995 A Laser-Doppler Velocimetry study of Ensemble Averaged Characteristics of the Turbulent Near Wake of A cylinder, J. of Fluid Mechanics, 304, pp. 285-319.

(2) Tennekes, H. and Lumley, J. L. 1972 A first Course in Turbulence, MIT Press, Cambridge, MA.

(3) Townsend, A. A. 1976 The Structure of Turbulent Shear Flow, Cambridge U. Press, Cambridge, UK.
(4) Van Dyke, M 1975 Perturbation Methods in Fluid Mechanics, Parabolic, Stanford, CA.

(5) White, F. M. 1986, Fluid Mechanics, McGraw-Hill, NY.

(6) Hughes, W. F and Brighton, J. A.,1991, Fluid Dynamics, $2^{\text {nd }}$ ed. McGraw-Hill Inc. NY.

(7) McCallen R., Couch R., Hsu J., Browand F., Hammache M., Leonard A., Brady M., Salari K., Rutledge W., Ross J., Storms B., Heineck JT., Driver D., Bell J., Zilliac G. 1999, Progress in reducing aerodynamic drag for higher efficiency of heavy duty trucks (class 7-8). SAE Paper 1999-01-223.

(8) Roy, C., McWherter-Payne, M., Solari, K. 2002, RANS simulations of a simplified Tractor/Trailer Geometry, UEF.

(9) Schlichting, H. 1979, Boundary Layer Theory, McGraw-Hill, New York.

(10)Haberman, R. 1983 Elementary Applied Partial Differential Equations with Fourier Series and Boundary value Problems, Prentice Hall, Englewood Cliffs, NJ.

(11)Logan, J. D. 1978 Applied Mathematics, Wiley, NY. 


\title{
APPENDIX F
}

\section{Commercial CFD Code Benchmarking for External Aerodynamics Simulations of Realistic Heavy-Vehicle Configurations}

\author{
Principal Investigator: W. David Pointer \\ Argonne National Laboratory \\ 9700 S Cass Avenue, NE-208, Argonne, IL 60439 \\ (630)252-1052; fax: (630)252-4500; email: dpointer@anl.gov \\ Technology Development Area Specialist: Sidney Diamond \\ (202) 586-8032; fax: (202) 586-1600; e-mail: sid.diamond@ee.doe.gov \\ Technical Program Manager: Jules Routbort \\ (630)252-5065; fax: (630)252-4289; email: routbort@anl.gov
}

Contractor: Argonne National Laboratory

Contract No.: W-31-109-ENG-38

\section{Objective}

- Evaluate capabilities in standard commercial computational fluid dynamics (CFD) software for the prediction of aerodynamic characteristics of a conventional U.S. Class 8 tractor-trailer truck.

- Develop "best practice" guidelines for the application of commercial CFD software in the design process of Class 8 vehicles.

\section{Approach}

- Develop computational models for the Generic Conventional Model (GCM) geometry.

- Benchmark the GCM simulations for computed aerodynamic drag force and pressure field distributions with NASA Ames $7 \mathrm{ft}$. by $10 \mathrm{ft}$. wind tunnel data.

\section{Accomplishments}

- Experimental measurements and computational predictions of the vehicle drag coefficient agreed within experimental uncertainty for the $0^{\circ}$ yaw simulations. Experimental measurements and computational predictions of the pressure distribution along the surface of the vehicle agree well everywhere except in the vicinity of the trailer base and underbody and the gap.

- Comparisons of computational predictions of the vehicle at a yaw angle of 10 degrees using coarse mesh models indicate that the accuracy of the steady RANS simulation capability is reduced at high yaw angles

\section{Future Direction}

- Extend evaluation of capabilities for prediction of aerodynamic drag in cases in which there is a cross-wind component (e.g., wind tunnel experiments where the vehicle is placed at some yaw angle) to fine mesh simulations and lower yaw angles.

- Consider alternate GCM configurations using various add-on devices to examine capabilities for the prediction of changes in drag coefficient

- Suggest potential drag reduction design options based on knowledge gained from computational effort. 


\section{Introduction}

In collaboration with the U.S. Department of Energy's Heavy Vehicle Aerodynamic Drag Consortium, Argonne National Laboratory is developing guidelines for the near-term use of existing commercial Computational Fluid Dynamics (CFD) tools by the heavy vehicle manufacturing industry. These guidelines are being developed based upon measured drag coefficients as well as detailed surface pressure distributions from wind tunnel experiments completed at NASA Ames Laboratory using a generalized 1/8th-scale conventional U.S. tractortrailer geometry, the Generic Conventional Model (GCM). ${ }^{1}$ Studies consider the effects of selection of global and near surface mesh size parameters and selection of turbulence modeling strategies.

\section{Selection of Commercial CFD Software}

The guidelines developed by this program are intended to be generic advice for the application of a commercial CFD software package for the prediction of heavy vehicle aerodynamic drag coefficients. Since this market is currently dominated by finite volume formulations, the guidelines will focus upon software using this methodology. Preliminary guideline development will be completed using the commercial CFD code Star-CD. ${ }^{2}$ The Star-CD software was selected for this purpose largely as a result of the flexibility in computational mesh development the code offers with the ability to utilize polyhedral "cut" cells and recognize both integral and arbitrary interfaces between regions of the computational domain. It is anticipated that the applicability of the general guidelines to other commercial CFD codes will be examined and that the extension of the guidelines to alternate commercial CFD software methodologies, such as LatticeBoltzmann, will be pursued following the initial development stage.

\section{The Generic Conventional Model}

The Generic Conventional Model (GCM) is a generalized representation of a conventional U.S. tractor-trailer truck developed by NASA Ames Research Center. The model is $1 / 8$ th scale, with approximate dimensions of $97 \mathrm{in}$. long by $13 \mathrm{in}$.

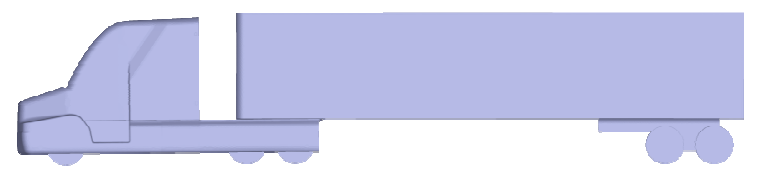

Figure 1. Generic Conventional Model (GCM) Standard Configuration

wide by $21 \mathrm{in.} \mathrm{high.} \mathrm{The} \mathrm{model} \mathrm{is} \mathrm{mounted} \mathrm{at} \mathrm{the}$ center of the ground plane of a $10 \mathrm{ft}$. wide by $7 \mathrm{ft}$. high wind tunnel test section. Instrumentation includes a force balance, 476 steady pressure transducers, 14 dynamic pressure transducers, and three-dimensional Particle Image Velocimetry (PIV). The nominal configuration (See Figure 1) is a representative model of a current-generation tractor-trailer truck.

\section{Computational Model}

The computational model employed in these studies was developed using the ES-Aero tool for aerodynamic drag simulation that is available as part of the Star-CD software package. The surface of the standard configuration GCM is defined using approximately 500,000 triangular surface elements based upon CAD data representations taken from optical scans of the actual model. The computational domain is developed based upon this surface definition using a semi-automated process that begins by creating a hexahedral mesh that is successively refined in smaller zones around vehicle, with 4 cell to 1 cell coupling employed at the interfaces between zones. The dimensions of hexahedral elements that make up the innermost zone are specified by user as the near vehicle cell size. The mesh elements near the vehicle surface are then further refined based upon local surface features identified by the user or selected automatically based on curvature or gap width. The user specifies a minimum allowable cell size that limits the refinement of the mesh in this step.

Using this locally refined hexahedral mesh, the original surface is "wrapped" by projecting the hexahedral mesh onto the original surface. In this manner, the multiple components defining the GCM are merged into a single surface. The "wrapped" surface definition is then volumetrically expanded to create a subsurface which is used to cut away the portions of the locally-refined hexahedral mesh that fall inside the vehicle. A brick and prism cell extrusion layer is 


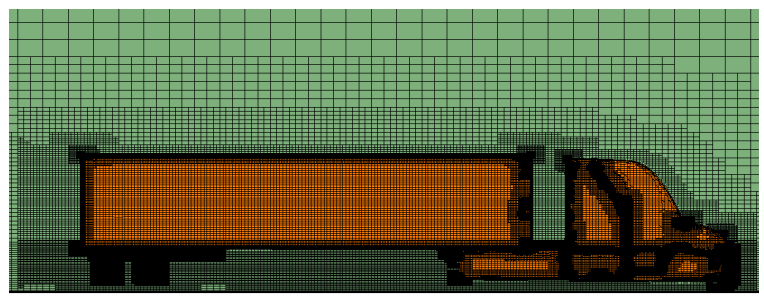

Figure 2. Example of computational mesh structure used in the simulation of the aerodynamic characteristics of Generic Conventional Model (GCM) configurations.

Table 1. Summary of computational domain characteristics for evaluation of bulk cell size effects.

\begin{tabular}{|c|c|c|c|}
\hline $\begin{array}{c}\text { Near } \\
\text { Vehicle } \\
\text { Cell Size } \\
(\mathrm{mm})\end{array}$ & $\begin{array}{c}\text { Minimum } \\
\text { Cell Size } \\
(\mathrm{mm})\end{array}$ & $\begin{array}{c}\text { Total } \\
\text { Number of } \\
\text { Volume } \\
\text { Elements }\end{array}$ & $\begin{array}{c}\text { Number of } \\
\text { Volume } \\
\text { Elements on } \\
\text { Surface }\end{array}$ \\
\hline 16.0 & 2.0 & 1012338 & 73574 \\
\hline 12.0 & 1.5 & 1737085 & 126119 \\
\hline 10.0 & 1.25 & 2345640 & 175105 \\
\hline 8.0 & 1.0 & 3282426 & 266666 \\
\hline 6.0 & 0.75 & 5695622 & 400382 \\
\hline
\end{tabular}

then created to fill the gap between the sub-surface and the "wrapped" surface. In this way, the nonhexahedral cut cells are removed some distance from the surface. A final step further refines the wake region and the underbody region in order to better capture important flow features in those regions. An example of the mesh construction of the computational domain used in the GCM simulations is shown in Figure 2.

When using locally-refined, partiallyunstructured computational domains with substantial numbers of non-hexahedral cells, the standard practice of evaluating grid convergence by uniformly refining the entire mesh in all directions becomes intractable. In the vehicle surface feature size based computational meshes used in these studies, two separate parameters determine the size of the mesh. The near vehicle cell size determines the bulk flow resolution surrounding the vehicle, and the minimum cell size determines the level of resolution allowed as a result of feature-based refinement around significant features of the vehicles surface. Mesh sensitivity analyses included in these studies examine the effects of changes in these parameters upon the prediction of the drag coefficient; however, this is not equivalent to the traditional grid convergence study for two reasons. First, the grid is not uniformly refined in all directions throughout the domain. Second, the vehicle surface definition cannot be exactly maintained for all models since the final surface definition is dependent upon the local refinement of the computational mesh.

\section{Bulk Flow Resolution}

Five unique computational domains were generated based upon the standard GCM configuration in order to evaluate the effects of the near vehicle cell size parameter on the prediction of the drag coefficient. Near-vehicle cell sizes of $16.0,12.0,10.0,8.0$ and $6.0 \mathrm{~mm}$ were considered. In each case, the minimum cell size resulting from local feature-based refinements is 12.5 percent of the near vehicle cell size and an additional restriction is set so that a minimum of 16 elements are required for the definition of a circle. In order to ensure that the quality of the vehicle surface is maintained, the cell layer immediately adjacent to the surface is refined to 25 percent of the original size prior to trimming. The computational domain characteristics are shown in Table 1.

Each computational domain was considered in a parametric study for evaluation of the sensitivity of the prediction of the vehicle drag coefficient to changes in the bulk flow resolution. A uniform velocity of $51.45 \mathrm{~m} / \mathrm{s}$, corresponding to a Reynolds number of $1.1 \times 10^{6}$ was specified at the inlet, and a zero gradient condition is specified at the outlet. In these simulations, the standard high Reynolds number k- $\varepsilon$ turbulence model and a logarithmic wall function are employed for prediction of turbulent kinetic energy and dissipation. 
For each case, 3000 iterations were calculated using Star-CD's standard conjugate gradient solver and the PISO predictor-corrector algorithm. Convergence criteria were set to insure that all cases would reach 3000 iterations before stopping. At the 3000th iteration, all residuals are less than $10^{-4}$. In addition to standard flow variable residual monitoring for the mass, momentum and energy equations, the drag coefficient of the vehicle is monitored as the solution develops to insure that the drag coefficient reaches a converged. Total computational time and clock time when using 16 processors for each simulation are shown in Table

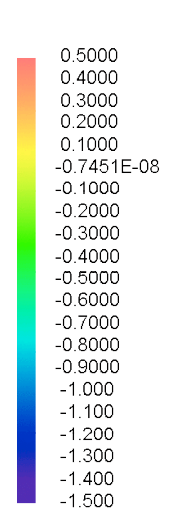

(a \& b)

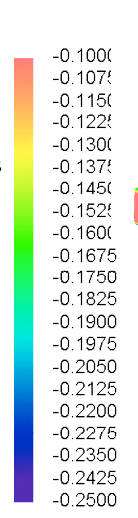

(c)

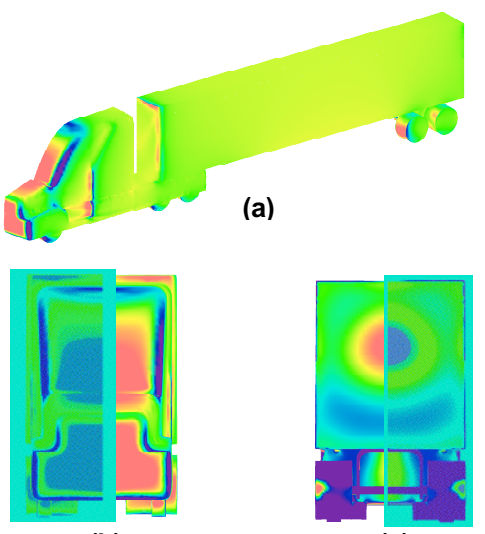

(b)

(c)
Figure 3. Predicted pressure coefficient distribution on the vehicle surface. Shown are (a) the side view of the full vehicle, (b) the front of the tractor, and (c) the base of the trailer.

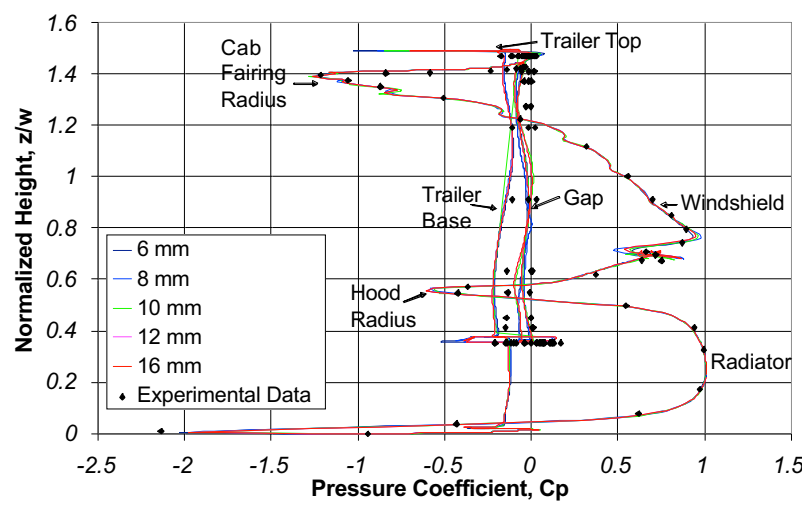

Figure 4. Comparison of predicted pressure coefficient distributions on the vehicle surface for various values of the near vehicle cell size parameter with experimental data for the GCM geometry.
Predicted drag coefficients from each of the five cases are compared with experimental data from wind tunnel tests in Table 3. While there is a trend of improvement with reduction in near vehicle cell size, the effects that lead to nonlinearity in the trend are not immediately clear. More detailed comparisons of pressure distributions on the surface of the vehicle provide better insight into the sensitivity of the predictive capability to the bulk flow resolution. The pressure coefficient distribution on the surface of the vehicle from the case using the mesh based upon an $8 \mathrm{~mm}$ near vehicle cell size is shown in Figure 3 as an example of a typical predicted pressure distribution on the vehicle surface. Pressure coefficient data was extracted along the centerline of the vehicle for each case and compared with experimental data as shown in Figure 4. These comparisons show that the difference in the predicted drag coefficient between models using different near vehicle cell sizes is a result of small differences in the pressure distribution over the entire surface rather than large localized differences.

\section{Near Wall Resolution}

Following the assessment of the effects of the near-vehicle cell size parameter on the accuracy of the drag coefficient prediction, the effect of the near-wall cell size parameter was also considered. The near-vehicle cell size was set to $8 \mathrm{~mm}$ and the minimum cell size for local refinement was reduced from $1 \mathrm{~mm}$ to $0.5 \mathrm{~mm}$. The change in the near wall resolution increases the number of computational elements from $3,282,426$ to $4,264,232$. When selecting near wall cell size limits, it is important to consider the appropriate limits of the parameter $y^{+}$, which describes the thickness of the region near the wall where the logarithmic law of the wall function is applied. For the turbulence model and wall function employed in these studies, the value of $\mathrm{y}+$ should fall between 20 and 200. A near-wall computational cell that is too small will result in a value of $y+$ that is too small, and a near-wall computational cell that is too large will result in a $\mathrm{y}+$ that is too large. As shown in Figure 5, the value of $y+$ falls within the appropriate range for the turbulence model employed. 
A simulation of the flow of air over the vehicle was completed using the refined computational mesh for comparison with the previous simulations. As in the previous cases, a uniform inlet velocity condition and a zero gradient outlet condition were specified, and the standard high Reynolds number k- $\varepsilon$ model was utilized. Convergence criteria were set so that

3000 iterations were completed, and all residuals fall below $10^{-4}$ by the 3000th iteration. The change in the computational mesh resolution results in an increase in the total CPU time from 610,958 seconds to 703,027 seconds.

The change in the near-wall refinement parameter results in a reduction in the error of the drag coefficient prediction from 4.2 percent to 1.0 percent, which is within experimental uncertainty. The predicted surface pressure distributions along

Table 2. Summary of computational cost for each case considered in the evaluation of bulk cell size effects.

\begin{tabular}{|c|c|c|}
\hline $\begin{array}{c}\text { Near- } \\
\text { Vehicle Cell } \\
\text { Size (mm) }\end{array}$ & $\begin{array}{c}\text { Total CPU } \\
\text { Time } \\
\text { (seconds) }\end{array}$ & $\begin{array}{c}\text { Total Clock } \\
\text { Time } \\
\text { (seconds) }\end{array}$ \\
\hline 16 & 206072 & 16454 \\
\hline 12 & 390113 & 29392 \\
\hline 10 & 417686 & 32182 \\
\hline 8 & 610958 & 44967 \\
\hline 6 & 2720956 & 188577 \\
\hline
\end{tabular}

Table 3. Effects of Near-Vehicle Cell Size Parameter on Accuracy of Drag Coefficient Prediction.

\begin{tabular}{|c|c|c|}
\hline $\begin{array}{c}\text { Near- } \\
\text { Vehicle Cell } \\
\text { Size (mm) }\end{array}$ & $\begin{array}{c}\text { Predicted } \\
\text { Drag } \\
\text { Coefficient }\end{array}$ & $\begin{array}{c}\text { Error in Drag } \\
\text { Coefficient }\end{array}$ \\
\hline experiment & 0.398 & 12.0 \\
\hline 16 & 0.449 & 10.3 \\
\hline 12 & 0.441 & 4.9 \\
\hline 10 & 0.418 & 4.2 \\
\hline 8 & 0.415 & 1.7 \\
\hline 6 & 0.405 & \\
\hline
\end{tabular}

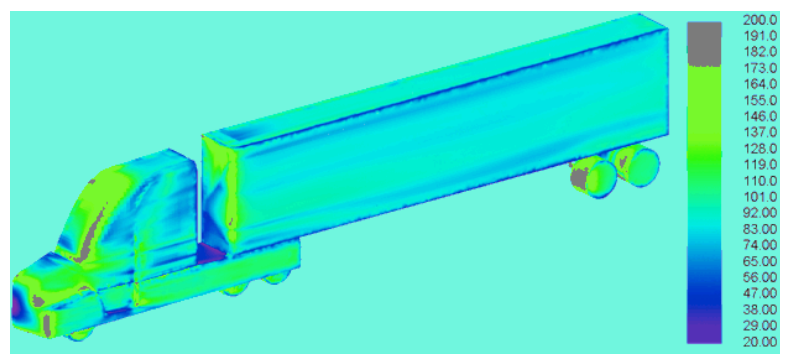

Figure 5. Values of the $y^{+}$parameter along the surface of the computational model of the GCM geometry when the computational mesh uses a near-vehicle cell size of $8 \mathrm{~mm}$ and a near-wall cell size limit of $1 \mathrm{~mm}$.

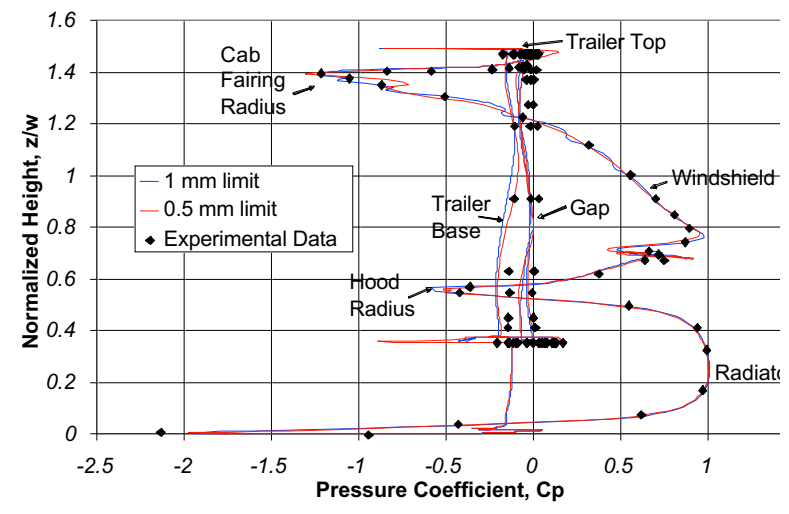

Figure 6. Comparison of predicted pressure coefficient distributions on the vehicle surface for various near wall cell size limits with experimental data for the GCM geometry.

the vehicle centerline for both cases are shown in Figure 6 along with the experimentally measured pressure distribution. As in the assessment of the effects of the near vehicle cell size parameter, comparisons of surface pressure data indicate that predicted drag coefficient between models using different near wall cell sizes is a result of small differences in the pressure distribution over the entire surface rather than large localized differences.

\section{Turbulence Model Selection}

In all simulations completed as part of the computational mesh sensitivity studies, the high Reynolds number k- $\varepsilon$ turbulence model was used in conjunction with a standard logarithmic wall function for the prediction of turbulent kinetic energy and eddy diffusivity. While the high Reynolds number k- $\varepsilon$ turbulence model is a 
robust general purpose turbulence model, the strong adverse pressure gradients and large flow recirculation regions associated with the GCM geometry may limit the applicability of steady state Reynolds Averaged Navier-Stokes (RANS) modeling strategies. Therefore, the sensitivity of the drag coefficient prediction to the choice of two equation steady RANS turbulence model was also assessed.

Using the computational mesh with a near vehicle cell size of $8 \mathrm{~mm}$ and a near wall cell size limit of $0.5 \mathrm{~mm}$, simulations of the aerodynamic characteristics of the GCM model were repeated using five steady RANS turbulence models and their associated wall functions:

1) the standard high-Reynolds number kmodel with logarithmic wall function

2) the Menter k- $\varepsilon$ SST model

3) the renormalization group (RNG) formulation of the $\mathrm{k}-\varepsilon$ model

4) the Chen formulation of the k- $\varepsilon$ model

5) the quadratic formulation of the k- $\varepsilon$ model

The standard k- $\varepsilon$ model and the k- $\varepsilon$ SST model are identical in the far field, but the k- $\varepsilon$ SST model incorporates additional detail in the near wall region and in separated flow regions. The RNG model is similar to the standard k- $\varepsilon$ model, but includes an additional term to account for the mean flow distortion of the dissipation. Chen's model is also similar to the standard k- $\varepsilon$ model, but includes an additional term to more effectively account for the effects of changes in the mean strain rate on the energy transfer mechanism of turbulence. The quadratic model is a higher order model of the k- $\varepsilon$ type that includes non-linear terms to allow for the anisotropy of turbulence in some flow fields.

As in the previous cases, a uniform inlet velocity condition and a zero gradient outlet condition were specified, and the standard high Reynolds number k- $\varepsilon$ model was utilized. Convergence criteria were set so that 3000 iterations were completed, and all residuals fall below $10^{-4}$ by the 3000 th iteration. The drag coefficients predicted using each of the selected steady-RANS turbulence models are shown in Table 4. More detailed comparisons of the predicted pressure coefficient distributions when
Table 4. Results of the evaluation of twoequation turbulence models for prediction of drag coefficients for the GCM geometry.

\begin{tabular}{|c|c|c|}
\hline Turbulence Model & $\begin{array}{c}\text { Predicted } \\
\text { Drag } \\
\text { Coefficient }\end{array}$ & $\begin{array}{c}\text { Percent } \\
\text { Error in } \\
\text { Prediction }\end{array}$ \\
\hline Experiment & 0.398 & -- \\
\hline $\begin{array}{c}\text { High-Reynolds } \\
\text { Number k-epsilon } \\
\text { Model }\end{array}$ & 0.402 & 1.0 \\
\hline $\begin{array}{c}\text { Menter k-e SST } \\
\text { model }\end{array}$ & 0.401 & 0.8 \\
\hline RNG model & 0.389 & 2.3 \\
\hline Chen's model & 0.3919 & 1.61 \\
\hline Quadratic model & 0.3815 & 4.32 \\
\hline
\end{tabular}

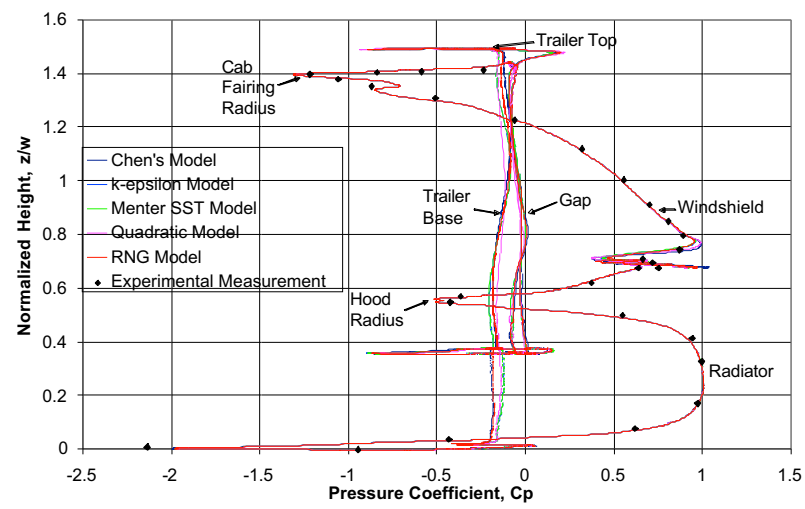

Figure 7. Comparison of predicted pressure coefficient distributions on the vehicle surface with experimental data for selected turbulence models.

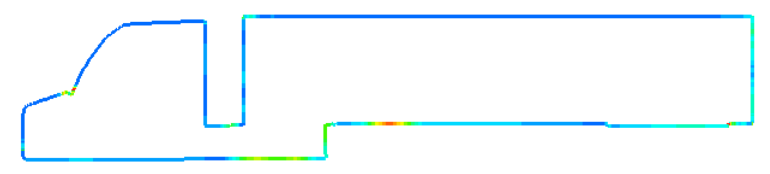

Figure 8. Standard deviation of the surface pressure distribution predictions using the selected turbulence models.

using each of the selected turbulence models are shown in Figure 7. Unlike previous studies, the differences in the predicted drag coefficient are largely a result of localized discrepancies in the surface pressure coefficient predictions in the regions of separated flow, as shown in Figure 8. 


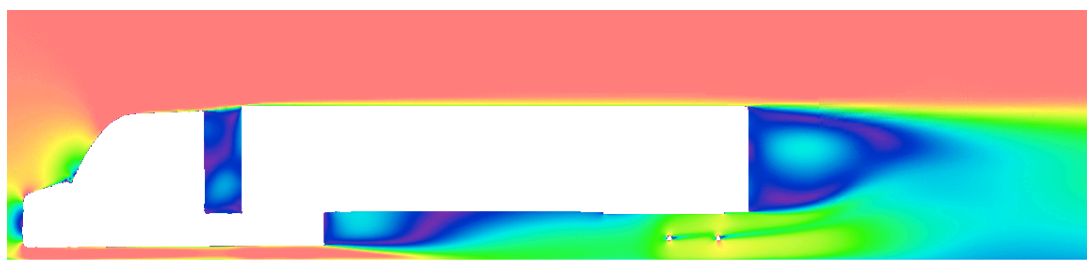

(a)

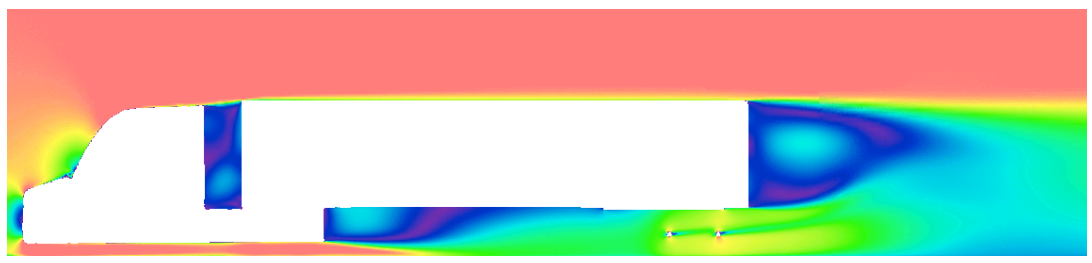

(b)

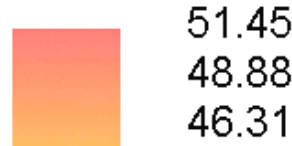

43.73

41.16

38.59

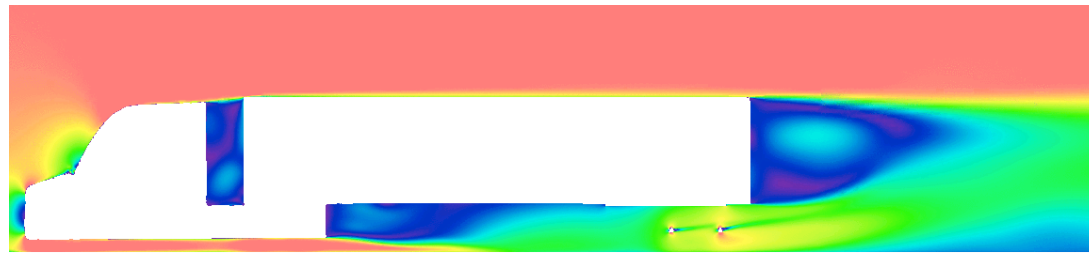

36.01

33.44

30.87

28.30

(c)

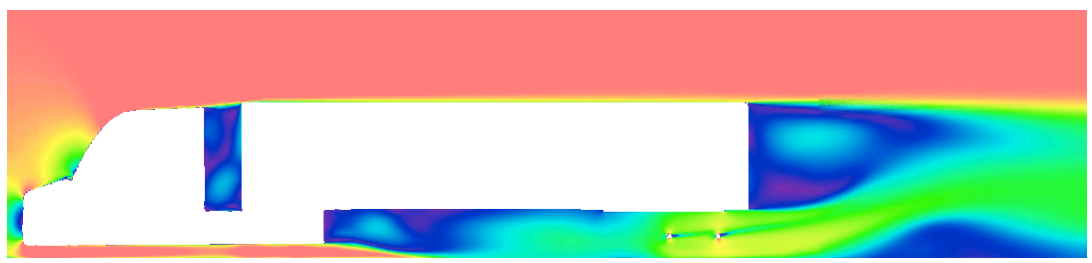

(d)

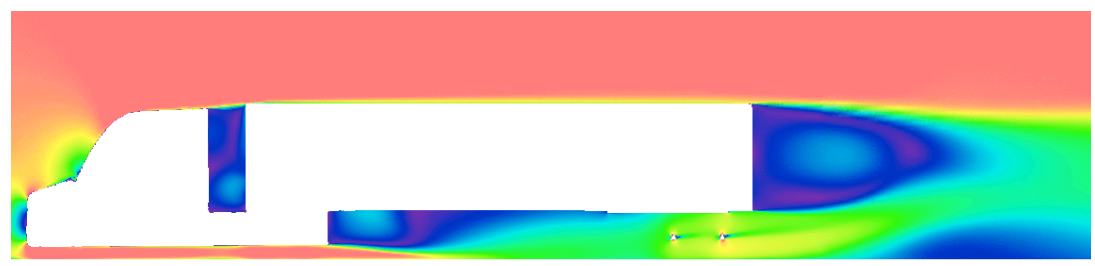

$0.9537 \mathrm{E}-0 \mathrm{E}$

(e)

Figure 9. Comparison of predicted steady state velocity fields for five selections of turbulence model: (a) the high-Reynolds number k- $\varepsilon$ model with logarithmic wall function, (b) the Menter k- $\varepsilon$ SST model, (c) the renormalization group (RNG) formulation of the k- $\varepsilon$ model, (d) the Chen formulation of the $\mathrm{k}-\varepsilon$ model, and (e) the quadratic formulation of the $\mathrm{k}-\varepsilon$ model. 
The differences in the predictions of the surface pressure distribution are a direct result of differences in the predicted flow fields. The predicted velocity magnitude at the centerline is shown for each selected turbulence model in Figure 9 . The primary differences in velocity field prediction occur in the recirculation zone under the trailer and in the interaction between the underbody flow and the separated flow region at the trailer base. The location of these differences corresponds to the largest discrepancies between the surface pressure distribution predictions.

\section{Full Vehicle versus Half Vehicle}

In order to evaluate the effects of considering only half of the vehicle rather than the full vehicle, two models were created using the full vehicle geometry. These models use the same mesh parameter settings as the two coarsest models considered in the mesh sensitivity study. The full vehicle models are based upon near-vehicle cell sizes of $12 \mathrm{~mm}$ and $16 \mathrm{~mm}$, with minimum nearwall cell sizes of $1.5 \mathrm{~mm}$ and $2.0 \mathrm{~mm}$ respectively. As in all previous studies, 3000 iterations were completed for each steady-state simulation and the convergence of the drag coefficient was monitored.

As shown in Table 5, drag coefficient predictions show a slight improvement in agreement with experimental measurements when the full-vehicle model is used. The comparison of more detailed pressure coefficient distributions along the vehicle surface, as shown in Figure 10, reveal that the most substantial discrepancies between the full and half vehicle model predictions occur along the underbody and in the gap between the tractor and trailer. The GCM geometry is in reality slightly asymmetric and the consideration of this geometric asymmetry is likely the primary difference in the models that contributes to these discrepancies.

\section{Vehicles at Yaw}

An assessment of the capabilities available in current generation commercial CFD software for the prediction of aerodynamic drag characteristics of heavy vehicles at yaw angles greater than zero is underway. A preliminary study using coarsemesh simulations of the GCM geometry at a yaw
Table 5. Comparison of drag coefficient predictions from half-vehicle and full-vehicle models.

\begin{tabular}{|c|c|c|}
\hline & \multicolumn{2}{|c|}{ Half-Vehicle } \\
\hline $\begin{array}{c}\text { Near-Vehicle } \\
\text { Cell Size (mm) }\end{array}$ & $\begin{array}{c}\text { Predicted Drag } \\
\text { Coefficient }\end{array}$ & $\begin{array}{c}\text { Percent Error in } \\
\text { Prediction }\end{array}$ \\
\hline 16 & 0.449 & 12.0 \\
\hline 12 & 0.441 & 10.3 \\
\hline & \multicolumn{2}{|c|}{ Full-Vehicle } \\
\hline 16 & $\begin{array}{c}\text { Predicted Drag } \\
\text { Coefficient }\end{array}$ & $\begin{array}{c}\text { Percent Error in } \\
\text { Prediction }\end{array}$ \\
\hline 12 & 0.441 & 10.3 \\
\hline & 0.426 & 6.7 \\
\hline
\end{tabular}

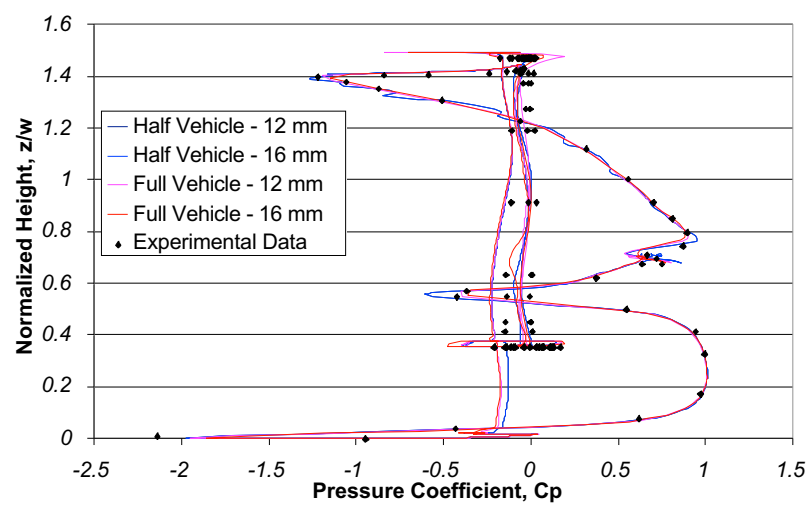

Figure 10. Comparison of predicted pressure coefficient distributions on the vehicle surface when the full vehicle model is used with predicted pressure coefficient distributions when the half vehicle model is used and with experimental data.

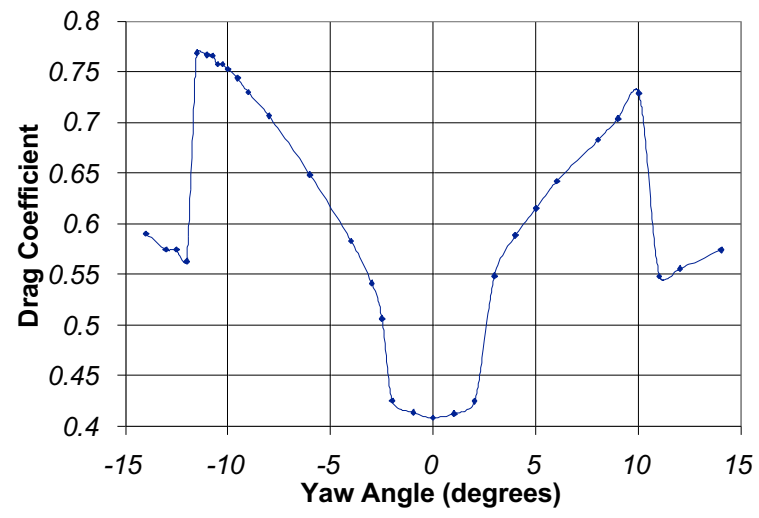

Figure 11. Experimental measurements of drag coefficients for the GCM vehicle geometry at yaw angles ranging from -14 to +14 
angle of ten degrees has been completed. The 10degree yaw angle was selected because as the yaw angle of the vehicle increases through 10-degrees, a changeover from a high drag state to a low drag state occurs (See Figure 11). The prediction of aerodynamic drag coefficients in this region is expected to be more difficult than at lower or higher yaw angles.

As with the no-yaw case, the study begins with an initial assessment of the sensitivity of the drag coefficient prediction to the base cell size in the near vehicle region. Since the full vehicle must be modeled when the vehicle is placed at a yaw angle other than zero, only coarse mesh models with near vehicle cell size parameters of $12 \mathrm{~mm}$ or $16 \mathrm{~mm}$ have been considered to date. As with the previous simulations, a total of 3000 iterations were completed for each simulation Results of these simulations are shown along with the zero yaw angle results in Figure 12. In going from a no-yaw condition of zero degrees to a yawed condition of 10 degrees, the error in the drag coefficient prediction increases from 10.3 percent 33.8 percent for the $16 \mathrm{~mm}$ case and from 6.7 percent to 23.5 percent in the $12 \mathrm{~mm}$ case.

Using the coarsest mesh, which is based upon a $16 \mathrm{~mm}$ near vehicle cell size, a preliminary assessment of the sensitivity of the prediction of drag coefficient to the selection of turbulence model has been completed. As in the assessment of steady RANS models for the no-yaw case, five two-equation type models were considered:

1) the high-Reynolds number k- $\varepsilon$ model

2) the Menter k- $\varepsilon$ SST model

3) the RNG formulation of the k- $\varepsilon$ model

4) the Chen formulation of the k- $\varepsilon$ model

5) the quadratic formulation of the k- $\varepsilon$ model

All five models use appropriate logarithmic wall functions to resolve the boundary layer region. Again, a total of 3000 iterations was completed for each simulation. Results of these simulations are shown in Table 6. The Mentor k- $\varepsilon$ SST model, which uses a k- $\varepsilon$ model in separated flow regions and a k- $\varepsilon$ model elsewhere, shows the greatest improvement over the standard high-Reynolds number k- $\varepsilon$ model. The Mentor k- $\varepsilon$ SST model also converges much more quickly than the other models, reaching the same level of convergence in fewer than half the iterations required when using

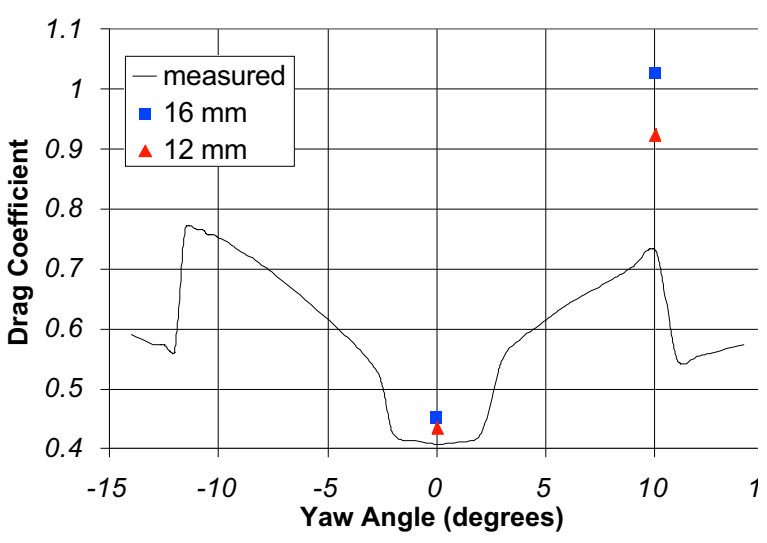

Figure 12. Comparison of computational predictions with experimental measurements of drag coefficients for models based upon near vehicle cell sizes of $16 \mathrm{~mm}$ and $12 \mathrm{~mm}$.

Table 6. Results of the evaluation of two-equation turbulence models for prediction of drag coefficients for the GCM geometry at a yaw angle of 10 degrees.

\begin{tabular}{|c|c|c|}
\hline $\begin{array}{c}\text { Turbulence } \\
\text { Model }\end{array}$ & $\begin{array}{c}\text { Predicted } \\
\text { Drag } \\
\text { Coefficient }\end{array}$ & $\begin{array}{c}\text { Percent } \\
\text { Error in } \\
\text { Prediction }\end{array}$ \\
\hline Experiment & 0.72955 & -- \\
\hline $\begin{array}{c}\text { High-Reynolds } \\
\text { Number k- } \\
\text { epsilon Model }\end{array}$ & 1.027 & 33.8 \\
\hline $\begin{array}{c}\text { Menter k-e SST } \\
\text { model }\end{array}$ & 0.844 & 14.5 \\
\hline RNG model & 1.014 & 32.6 \\
\hline Chen's model & 1.052 & 36.3 \\
\hline Quadratic model & 1.001 & 31.3 \\
\hline
\end{tabular}

other models. The velocity field for this simulation is shown in Figure 13.

\section{Fine Mesh Models for Full Vehicles}

The number of cells used by the semiautomatic meshing tool in the process of creating the final computational mesh increases rapidly as the near vehicle cell size is reduced. Consequently a 64-bit computational platform with significant memory available to the processors running the meshing tools is needed to construct the fine mesh models of the full truck geometry, both at no yaw and a yaw angle of 10 degrees. The Star-CD software has been installed on a 64-bit Itanium 2 workstation with $24 \mathrm{~GB}$ of RAM to allow the 
construction of more refined computational models. A significant effort has been dedicated to identifying and addressing associated with this new port of the software. It is anticipated that assessments using fine meshes will be completed in early FY 2005.

\section{Future Work}

Ongoing efforts will continue to focus on the assessment of the capabilities within current generation software using simple steady RANS modeling strategies for the prediction of changes in drag with changes in geometry or flow conditions. These efforts will consider the standard configuration of the GCM geometry at additional yaw angles, as well as the alternate configurations of the GCM geometry shown in Figure 1. Upon completion of computational studies for each configuration of the geometry, predictions of drag coefficient and surface pressure distributions will be compared with experimentally measured values for that configuration.

\section{Conclusions}

These studies are the initial component of an assessment of the capabilities for the prediction of heavy vehicle aerodynamic characteristics using current generation commercial computational fluid dynamics software. Based upon the outcomes of these studies, guidelines are being developed for the immediate application of these current generation tools by the heavy vehicle manufacturing community. Initial assessments have shown that full body drag coefficients can be predicted within less than 1 percent of the measured value, which is within experimental accuracy. The surface pressure distributions can be predicted with reasonable accuracy using fine mesh models. The coarsest mesh model of the full vehicle provides a prediction of the drag coefficient within 11 percent of the measured value. When the vehicle is yawed to 10 degrees, the coarsest model provides a prediction within 19 percent of the measured value if the Menter k- $\varepsilon$ SST model is used.

\section{References}

${ }^{1}$ Satran, D., "An Experimental Study of the Generic Conventional Model (GCM) in the NASA Ames 7-by-10-Foot Wind Tunnel," United Engineering Foundation Conference on The Aerodynamics of Heavy Vehicles: Trucks, Buses, and Trains, United Engineering Foundation, New York, 2002.

${ }^{2}$ Star-CD, version 3.150A, CD-Adapco Group, Melville, NY. 


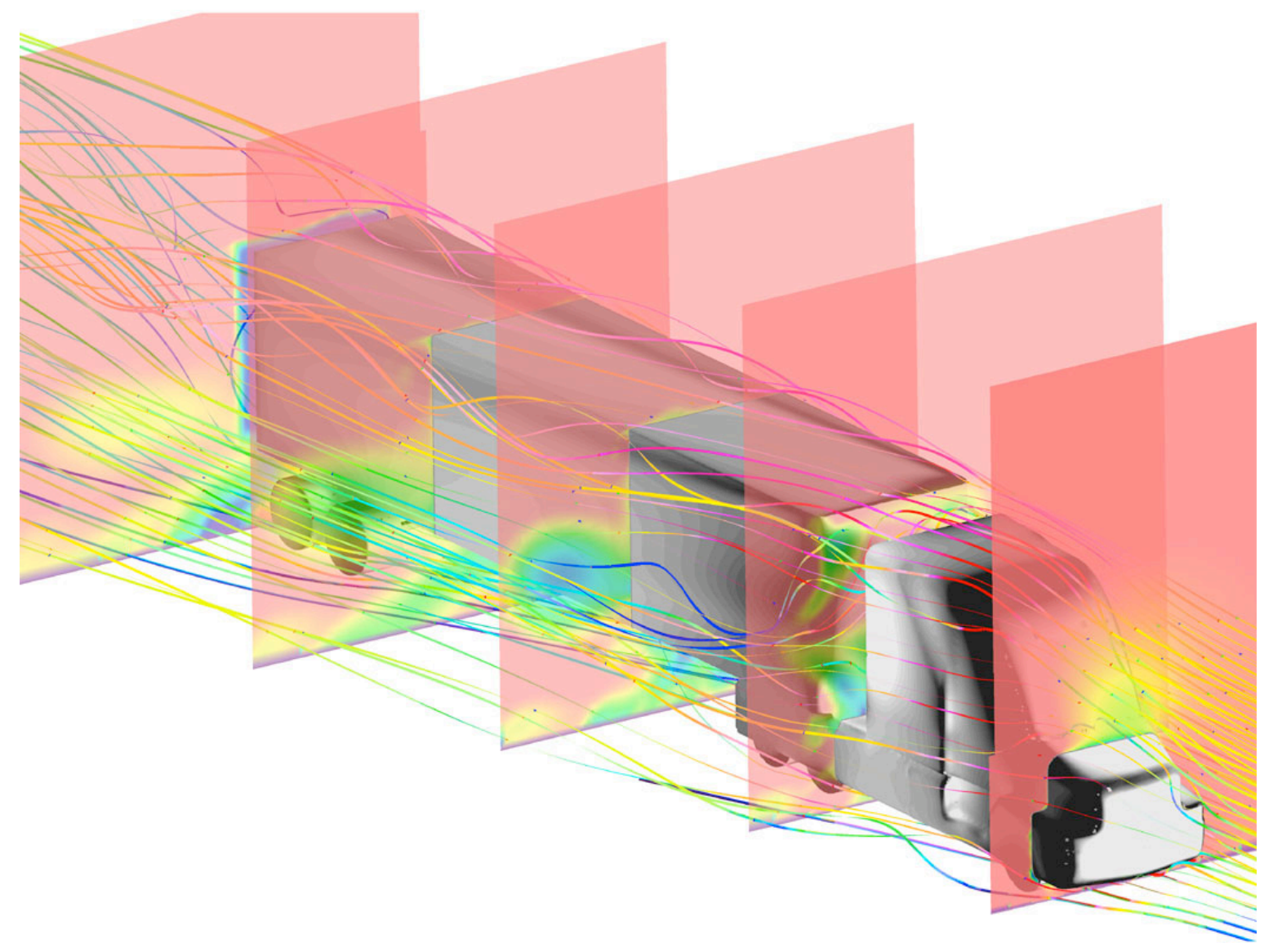

Figure 13. Predicted streamlines across the surface of the GCM geometry when the vehicle is placed at a yaw angle of 10 degrees. 


\title{
APPENDIX G
}

\section{Bluff Body Flow Simulation using a Vortex Element Method}

\author{
Principal Investigator: Anthony Leonard \\ California Institute of Technology \\ 1200 E. California Blvd \\ Pasadena CA 91125 \\ (626) 395-4465,fax: (626) 577-9646, tony@galcit.caltech.edu
}

Technology Development Area Specialist: Sidney Diamond

(202) 586-8032; fax: (202) 586-1600; e-mail: sid.diamond@ee.doe.gov

Technical Program Manager: Jules Routbort

(630)252-5065; fax: (630)252-4289; email: routbort@anl.gov

\section{Participants:}

Michael Rubel, Caltech, mrubel@galcit.caltech.edu

Philippe Chatelain, Caltech, philch@galcit.caltech.edu

Contractor: California Institute of Technology

Contract No.: DE-AC03-98EE50506

\section{Objective}

- Study application of vortex particle methods to complex truck geometries at high Reynolds numbers

- Investigate Large Eddy Simulation (LES) in the context of such solutions.

\section{Approach}

- Develop physically and mathematically correct treatments for the generation of vorticity at complex boundaries.

- Extend boundary treatment to cases of spinning bodies such as tires

- Reduce the computational work required to arrive at solutions by novel time integration techniques

- Reconcile LES theory with this framework

\section{Accomplishments}

- Implemented near-wall vorticity elements with wall stress evaluation and additional Biot-Savart term for spinning objects

- Performed preliminary simulations of spinning sphere flows at $\mathrm{Re}=300$ for a dimensionless spin rate of 0.5 and 2 spin angles

- Implemented a multiscale time integration algorithm with clear order of accuracy and convergence properties

- Developed a new ensemble-averaging theory for LES

\section{Future Direction}

- Computation of flows around tumbling objects and spinning tires

- Perform detailed performance measurements on the multiscale time integrator, extend to parallel computation 
- Test ensemble averaging theory on turbulent flows, ascertain whether the theory will in fact be able to predict LES parameters

\section{Introduction}

Heavy ground vehicles, especially those involved in long-haul freight transportation, consume a significant part of our nation's energy supply. It is therefore of utmost importance to improve their efficiency, both to reduce emissions and to decrease reliance on imported oil.

At highway speeds, more than half of the power consumed by a typical semi truck goes into overcoming aerodynamic drag, a fraction which increases with speed and crosswind. Thanks to better tools and increased awareness, recent years have seen substantial aerodynamic improvements by the truck industry, such as tractor/trailer height matching, radiator area reduction, and swept fairings. However, there remains substantial room for improvement as understanding of turbulent fluid dynamics grows.
Our group's research effort focuses on vortex particle methods, a novel approach for computational fluid dynamics (CFD). Where common CFD methods solve or model the NavierStokes equations on a grid which stretches from the truck surface outward, vortex particle methods solve the vorticity equation on a Lagrangian basis of smooth particles and do not require a grid.

We are working to advance the state of the art in vortex particle methods, improving their ability to handle the complicated, high Reynolds number flow around heavy vehicles. Specific challenges that we have addressed in the past year include finding strategies to accurately capture vorticity generation and resultant forces at the truck wall, handling the aerodynamics of spinning bodies such as tires, computation time reduction through improved integration methods, and theoretical
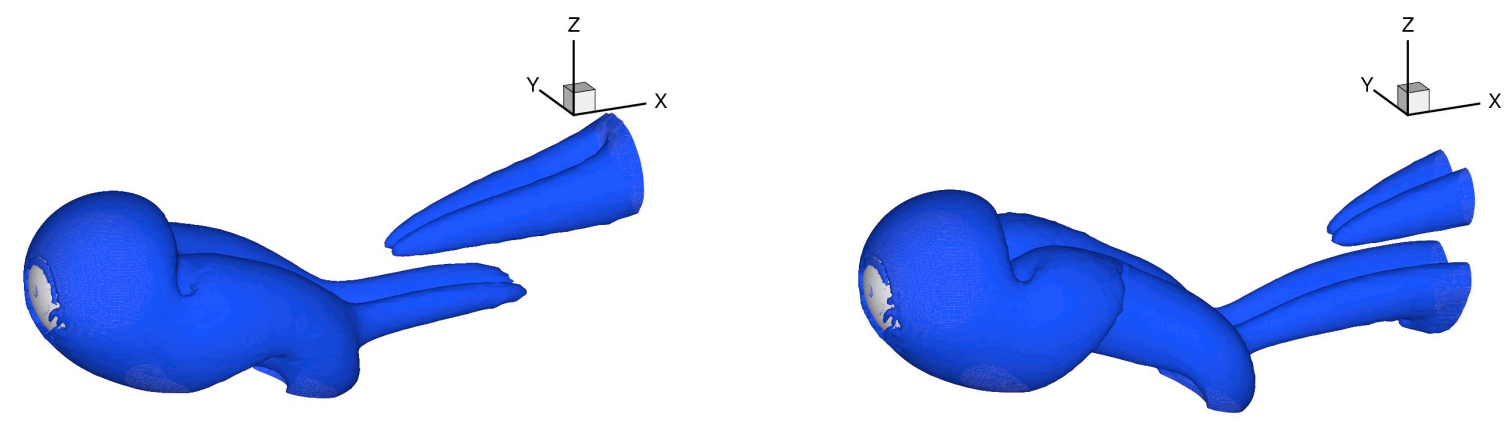

$$
\mathrm{T}=6
$$

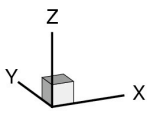

$$
\mathrm{T}=7.125
$$
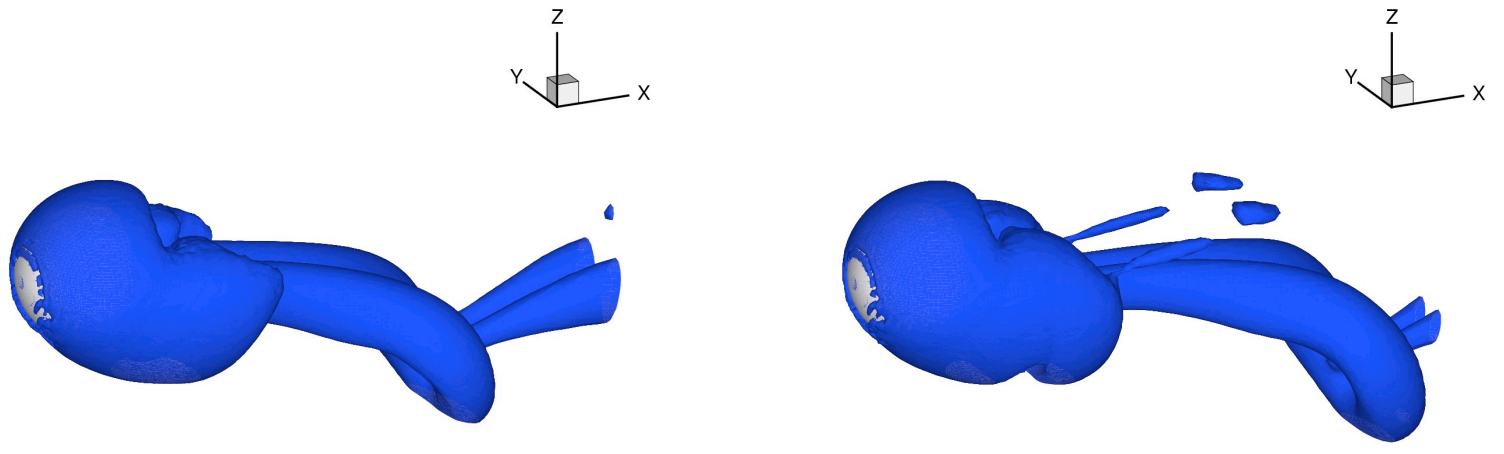

$$
\mathrm{T}=8.25
$$

$$
\mathrm{T}=9.375
$$

Figure 1 Spinning sphere at $\mathrm{Re}=300$, span-wise rotation (axis of rotation is perpendicular to free stream direction): vorticity structures ( $\mathrm{q}=0$ surfaces) 
treatment work on large eddy simulation (LES)

\section{Near wall vorticity}

We developed a representation of near-wall vorticity by means of an attached regularized sheet. This sheet has several roles. It interacts viscously with the rest of the flow, receives contributions of elements close to the wall during a redistribution and helps in capturing the high vorticity gradients near the wall.

This last role is critical if one needs to accurately measure stresses at the wall. In addition, we introduce a correction that takes into account the gradient of vorticity, which can be estimated from the solution of the panel solver.

\section{Spinning boundaries}

The flow around spinning objects is of particular interest because it is encountered around turbulence modeling.

the wheels of heavy vehicles and will interact with the rest of the flow. It is also interesting because of its impact on the problem of splash-and-spray. Because we use a vorticity based formulation along with a computation of velocities by BiotSavart, we need to account for the vorticity inside any rotating object. This term, a volume integral, is not the best suited for our method which uses a surface mesh to represent boundaries. We thus switch to a surface integral by application of Gauss's theorem.Preliminary results for two configurations involving a spinning sphere were accomplished in FY2004; the rotation axis of the sphere was aligned with the stream or set perpendicular to it. Both cases were computed for $\mathrm{Re}=300$ and a dimensionless spin velocity $\mathrm{WR} / \mathrm{U}_{\infty}$ of 0.5 , where $\mathrm{W}$ is the angular velocity. The case of a perpendicular axis is of particular interest
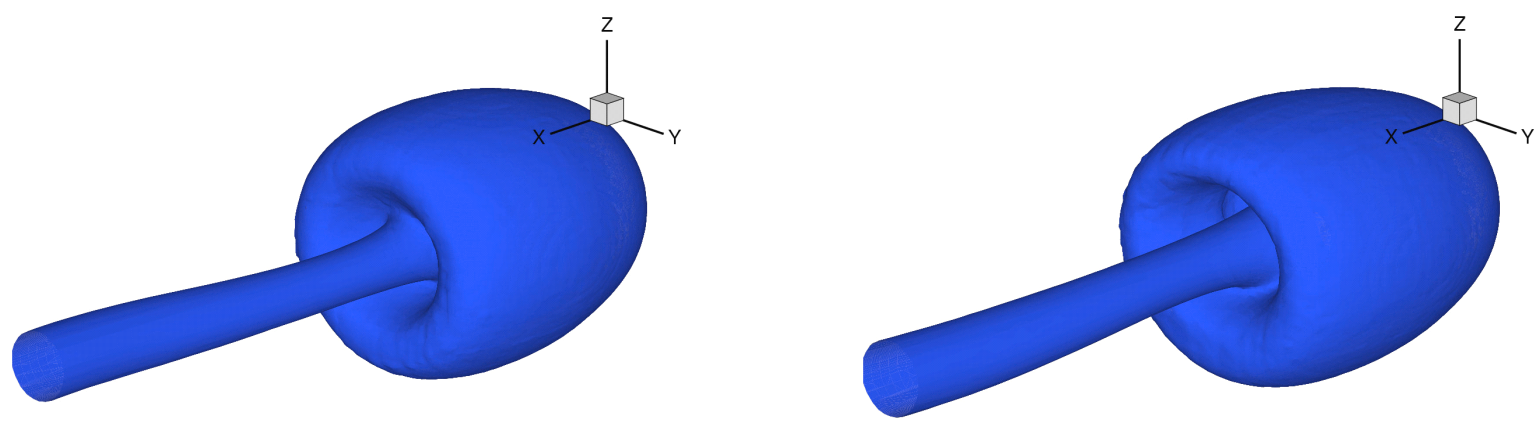

$$
\mathrm{T}=7
$$

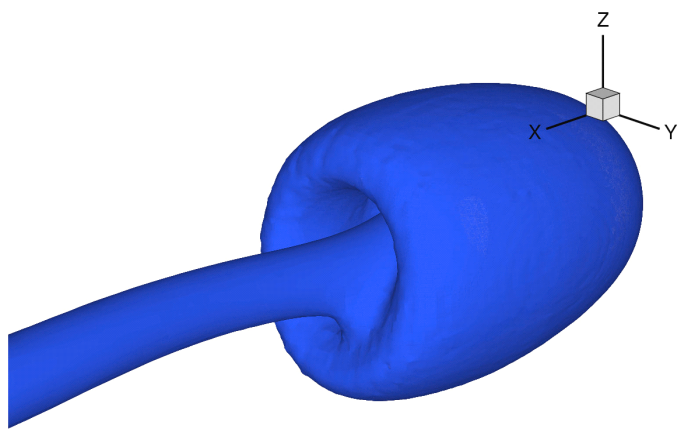

$$
\mathrm{T}=11
$$

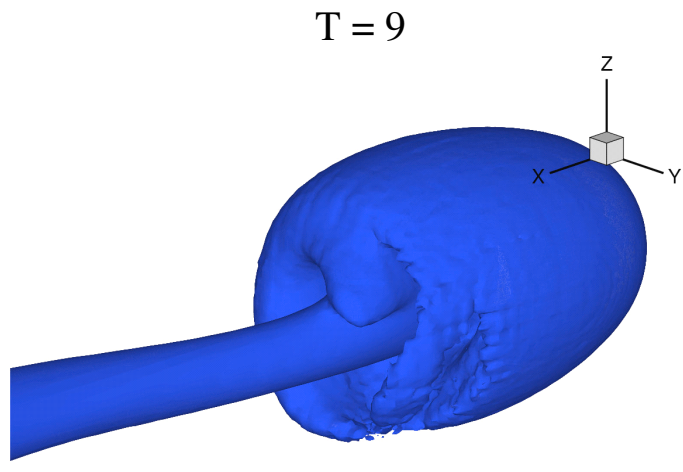

$$
\mathrm{T}=13
$$

Figure 2 Spinning sphere at $\mathrm{Re}=300$, stream-wise rotation(axis of rotation is aligned with free stream direction): vorticity structures ( $\mathrm{q}=0$ surfaces) 


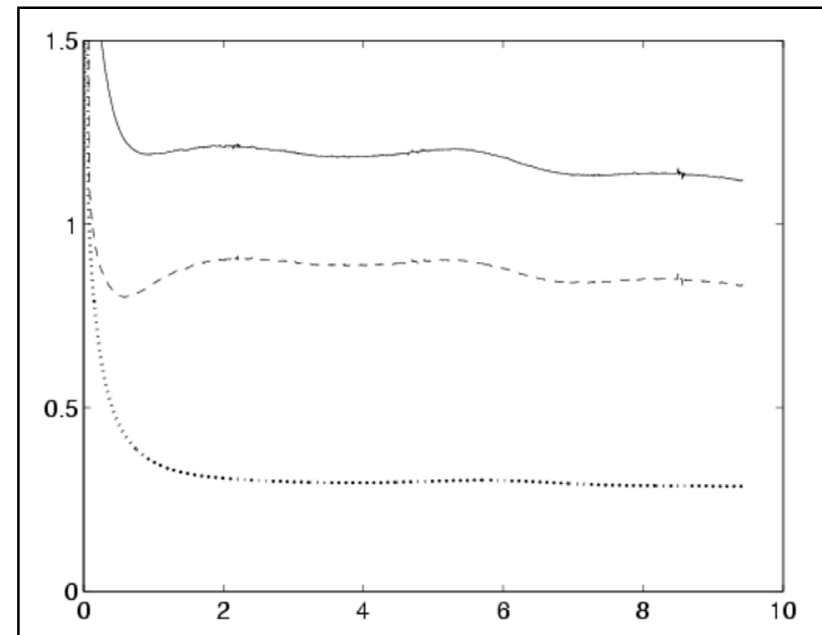

Figure 3 Spinning sphere at $\operatorname{Re}=300$, spanwise rotation: drag coefficient, shear component (dotted), pressure component (dashed), total (solid) versus time

because of its resemblance to the geometry of a spinning tire. The problem is not symmetric and does not need to be perturbed to trigger shedding; Figure 1 shows vortex structures identified by contours of $Q=-\frac{1}{2} \operatorname{Tr}(\nabla u \cdot \nabla u)$.

Even though the problem is still marked by the initial transient, the sphere sustains an important amount of lift (Figure 4, Figure 3) and develops a wake that comprises a system of counter-rotating vortices.

The stream-wise rotation is also of interest because of the various wake structures that are observed across the ranges of Reynolds numbers and spin rates. Our results show the transition from the axi-symmetric wake typical of a low Reynolds number to an asymmetric periodic wake (Figure 2).

\section{Timestepping}

Because contemporary CFD is limited by the power of available computers, it is of interest to reduce the work necessary to compute a given flow. One major area of inefficiency which remains largely untapped is the time integration process.

In Figure 5, one sees a frequency distribution of the strengths of vortex particles from one snapshot of a very low Reynolds number (1000) truck model simulation. By dimensional analysis,

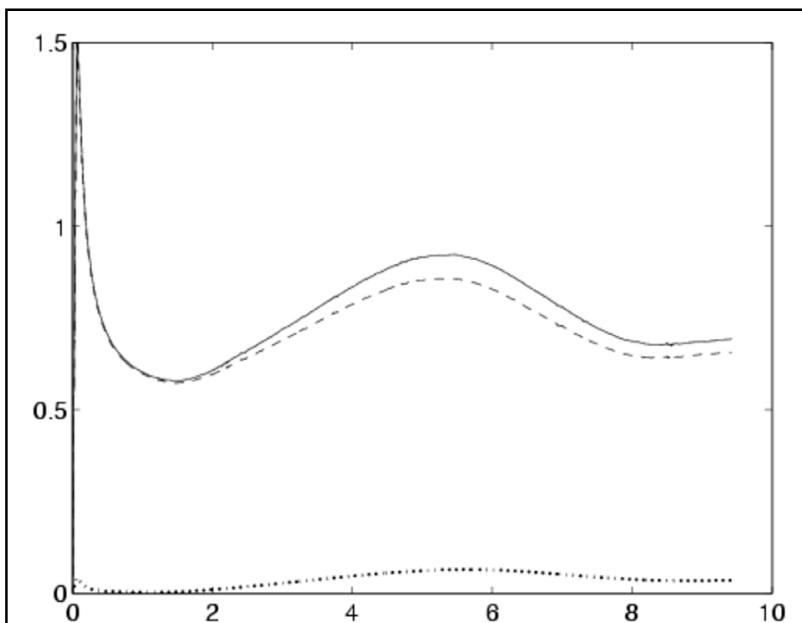

Figure 4 Spinning sphere at $\operatorname{Re}=300$, spanwise rotation: lift coefficient, shear component (dotted), pressure component (dashed), total (solid) versus time

the local timescale is inversely proportional to the local strain rate tensor norm, which for the purpose of this illustration is taken to be particle strength (a choice which is approximate in that it neglects the symmetric part of the tensor). In a conventional timestepper, even an adaptive one, the CFL condition limits integration rate according to the strongest gradient in the flow. However, even at this unrealistically small Reynolds number, the mean strength is hundreds or thousands of times smaller than that of the strongest particles, so most of the flow is being over-resolved by the same factor. Performing timesteps which are adaptive per-particle, rather than per-step, could potentially reduce the computational workload by orders of magnitude.

Some multiscale integration techniques are available, but are not suitable for vortex-based fluid flow problems, which operate over a continuous range of scales and involve fairly complicated tree-based right-hand-side evaluation. The goal of this phase of research has been development of a new multiscale time integration scheme which is tailored to vortex particle methods.

Such a method has been developed and refined over the course of several years, and is now beginning to bear fruit. In Figure 6, one sees in the left column several snapshots of a simple vortex particle flow developing in two dimensions, with corresponding particle-specific timesteps on 


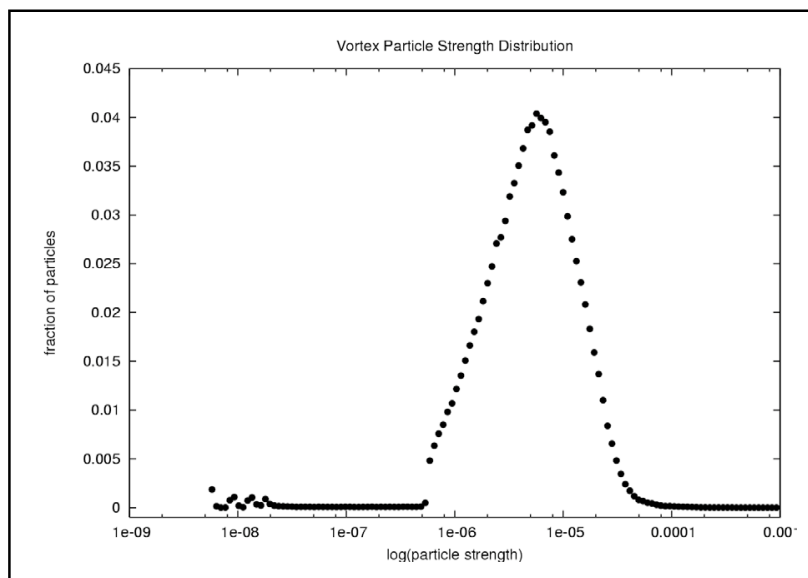

Figure 5 Vortex particle strength distribution around GTS truck model

the right. The most significant challenge in developing the method was achieving decent scaling for large numbers of particles; the latest incarnation scales linearly with the total number of timesteps across all particles, as required.

Rigorous order-of-accuracy estimates have been derived (the method can be made accurate to any order) and a number of successful tests have been performed, though more will be required. A paper detailing the method is in progress.

\section{Theoretical LES work}

There is ongoing debate on the relationship of large eddy simulation (LES) and Reynoldsaveraged Navier-Stokes (RANS) solutions to the filtered or time-averaged direct numerical simulations (DNS) they are designed to model. Due to the chaotic nature of turbulence, the modeled solution is not generally the same result one would obtain by applying its simplifying assumption to an exact solution. The problem is not merely an academic one; understanding how a model relates to the flow being modeled is essential for choosing parameters correctly, which in turn is essential for finding and interpreting computed turbulent flows in the context of heavy vehicle aerodynamics.

We are investigating the implications of a new theoretical concept which treats LES as an explicit ensemble averaging procedure. This is still a new idea, but there is hope that it will be applicable to choosing parameters for LES models. It has been

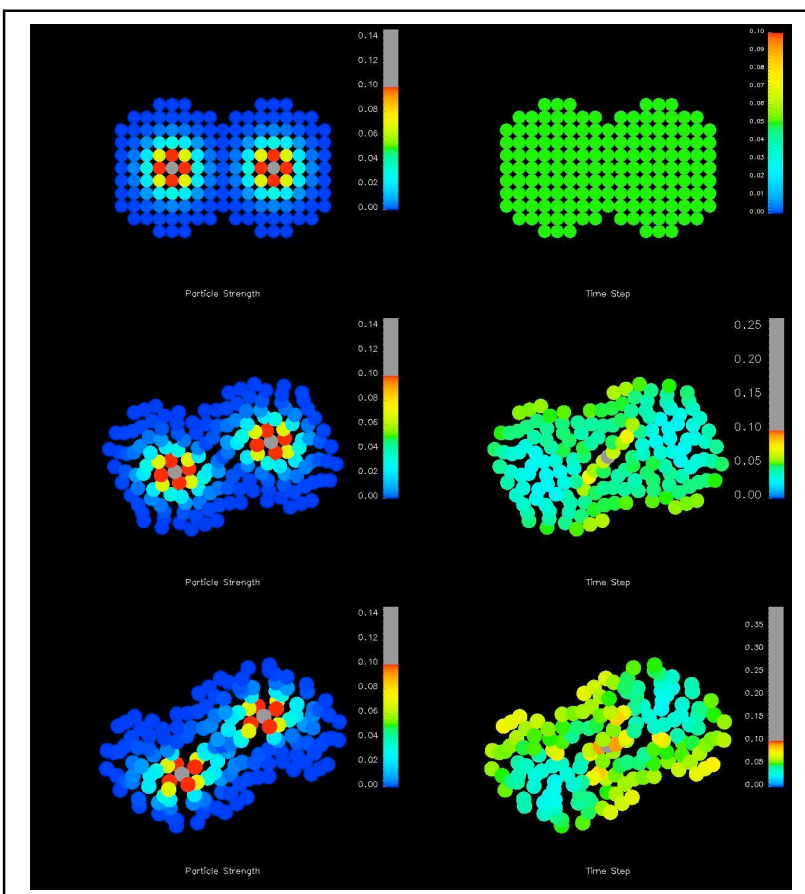

Figure 6 Stages in asynchronous time integration of two vortex patches: strengths (left) and timesteps (right)

tested to an extent on the Lorenz equations. The first nontrivial test on 1-D Burgers' equation is expected to be complete soon; if the test works, the method should be straightforward to extend to 3-D Euler and Navier-Stokes equations.

\section{Conclusions}

Vortex method development continues mostly according to plan; developments in FY2004 mean it should now be possible to simulate complicated flows around truck bodies, including those around rotating tires. Time integration techniques have improved, although these improvements are not yet backported into the main code. Work began on development of Large Eddy Simulation ensemble theory, and preliminary tests to prove or disprove its usefulness will be conducted in the near future. 